Métodos numéricos para o controle linear quadrático com saltos e observação parcial de estado

\author{
Daiane Cristina Bortolin
}



SERVIÇO DE PÓS-GRADUAÇÃO DO ICMC-USP

Data de Depósito: 16 de março de 2012

Assinatura:

\title{
Métodos numéricos para o controle linear quadrático com saltos e observação parcial de estado
}

\author{
Daiane Cristina Bortolin
}

Orientador: Prof. Dr. Eduardo Fontoura Costa

\begin{abstract}
Dissertação apresentada ao Instituto de Ciências Matemáticas e de Computação - ICMC-USP, como parte dos requisitos para obtenção do título de Mestre em Ciências - Ciências de Computação e Matemática Computacional. VERSÃO REVISADA.
\end{abstract}


Ficha catalográfica elaborada pela Biblioteca Prof. Achille Bassi e Seção Técnica de Informática, ICMC/USP, com os dados fornecidos pelo(a) autor(a)

Bortolin, Daiane Cristina
M739m
quadrático com saltos e observação parcial de estado
/ Daiane Cristina Bortolin; orientador Eduardo
Fontoura Costa. -- São Carlos, 2012.
117 p.
Dissertação (Mestrado - Programa de Pós-Graduação en
Ciências de Computação e Matemática Computacional) --
Instituto de Ciências Matemáticas e de Computação,
Universidade de São Paulo, 2012.
Tisteoria de controle. 2. Sistemas lineares. 3.
Tistemas estocásticos. 4. Sistemas com saltos
markianos. I. Costa, Eduardo Fontoura, orient. II.
Título.


Aos meus pais

Adélia e Roberto. 



\section{Agradecimentos}

Ao Prof. Dr. Eduardo Fontoura Costa pela orientação, pela dedicação e atenção dispensada durante todo o desenvolvimento deste trabalho.

Ao Carlos Alexandre Silva pela sua contribuição neste trabalho.

Aos meus pais Adélia e Roberto e aos meus irmãos Danilo e Diego por todo amor, carinho e suporte.

Ao Marciel Alberto Gomes por tudo o que passamos juntos nesses anos. Pela sua amizade, seu carinho e paciência.

Ao Conselho Nacional de Desenvolvimento Científico e Tecnológico (CNPq) pelo apoio financeiro.

A todos os colegas, professores e funcionários do Instituto de Ciências Matemática e de Computação da Universidade de São Paulo. 

BORTOLIN, D. C.. Métodos numéricos para o controle linear quadrático com saltos e observação parcial de estado. 2012. 117p. Dissertação (Mestrado) - Instituto de Ciências Matemáticas e de Computação, Universidade de São Paulo, São Carlos, 2012.

Este trabalho consiste no estudo de métodos de otimização aplicados em um problema de controle para sistemas lineares com saltos markovianos (SLSM). SLSM formam uma importante classe de sistemas que têm sido muito úteis em aplicações envolvendo sistemas sujeitos a falhas e outras alterações abruptas de comportamento. Este estudo enfoca diferentes métodos para resolução deste problema. Comparamos o método variacional com o de Newton, sob o ponto de vista do número de problemas resolvidos e pelo nível de sub-otimalidade obtido (relação entre os custos obtidos por estes métodos). Também propomos um novo método, o qual pode ser inicializado com soluções de equações de Riccati acopladas, e o comparamos com o método variacional. Além disso, para a comparação dos métodos, propomos um algoritmo que gerou dez mil exemplos.

Palavras-chave: Teoria de controle. Sistemas lineares. Sistemas estocásticos. Sistemas com saltos markovianos. 

BORTOLIN, D. C.. Numerical methods for linear quadratic control with jump and partial state observation. 2012. 117p. Dissertação (Mestrado) - Instituto de Ciências Matemáticas e de Computação, Universidade de São Paulo, São Carlos, 2012.

This work addresses optimizations methods applied to a control problem for linear systems with markovian jumps, which form an important class of systems that have been very useful in applications involving systems subject to failures and other abrupt changes. This study focuses on different methods for solving this problem. We compare the variational approach with the Newton method, in terms of the number of solved problems and the level of sub-optimality (ratio between the costs obtained by these approaches). We also propose a new method, which can be initialized with solutions of coupled Riccati equations, and we compare it with the variational approach. We have proposed an algorithm for creating ten thousand examples for the comparisons.

Keywords: Control theory. Linear systems. Stochastic systems. Markov jump systems. 



\section{Lista de Figuras}

2.1 Diagrama de estados . . . . . . . . . . . . . . . . . . . . 24

2.2 Realizações de $x_{k}^{\prime} x_{k}$, sua média e o valor esperado $E\left\{x_{k}^{\prime} x_{k}\right\} \ldots \ldots 26$

3.1 Resultados do método variacional para o Exemplo $3.1 \ldots$. . . . . . . 33

4.1 Resultados do método de Newton modificado para o Exemplo 4.1 . . . 43

4.2 Resultados do método de Newton modificado para o Exemplo 4.2 . . . 45

4.3 Resultados do método de Newton modificado para o Exemplo 4.3 . . . 46

5.1 Resultados da estratégia de observação indireta para o Exemplo 5.1 . . 59

5.2 Resultados da estratégia de observação indireta para o Exemplo 5.2 . . 61

6.1 Quantidade de problemas versus o valor de $\alpha \ldots \ldots \ldots$. . . . . 68

6.2 Resultados obtidos por cada método, na Classe 1 . . . . . . . . . . . 69

6.3 Comparação dos resultados de cada método, para a Classe 1 . . . . . 69

6.4 Custo do MV versus o da EOI (MV), para a Classe $1 \ldots \ldots$. . . . 70

6.5 Tempo de CPU do MV versus o da EOI (MV), para a Classe 1 . . . . 71

6.6 Resultados obtidos por cada método, para a Classe $2 \ldots \ldots$. . . . 72

6.7 Comparação dos resultados de cada método, para a Classe 2 . . . . . 73

6.8 Custo do MV versus o da EOI (MV), para a Classe $2 \ldots \ldots$. . . . 73

6.9 Tempo de CPU do MV versus o da EOI (MV), para a Classe $2 \ldots \ldots 74$

6.10 Resultados obtidos por cada método, para a Classe 1 . . . . . . 76

6.11 Comparação dos resultados de cada método, para a Classe 1 . . . . 76

6.12 Custo do MV versus o do MN, para a Classe $1 \ldots \ldots$. . . . . . 77

6.13 Tempo de CPU do MV versus o do MN, para a Classe $1 \ldots \ldots$. . . . 78

6.14 Resultados obtidos por cada método, para a Classe 2 . . . . . . . 79

6.15 Comparação dos resultados de cada método, para a Classe 2 . . . . . 80 
6.16 Custo do MV versus o do MN, para a Classe $2 \ldots . . . .80$

6.17 Tempo de CPU do MV versus o do MN, para a Classe 2 . . . . . . . . 81

6.18 Resultados obtidos por cada método, na Classe 1 . . . . . . . . . . 83

6.19 Comparação dos resultados de cada método, para a Classe 1 . . . . . . 83

6.20 Custo do MV versus o da EOI (MN), para a Classe 1 . . . . . . . . . 84

6.21 Tempo de CPU do MV versus o da EOI (MN), para a Classe 1 . . . . 85

6.22 Resultados obtidos por cada método, para a Classe 2 . . . . . . . 86

6.23 Comparação dos resultados de cada método, para a Classe 2 . . . . . 86

6.24 Custo do MV versus o da EOI (MN), para a Classe $2 \ldots$. . . . . . . 87

6.25 Tempo de CPU do MV versus o da EOI (MN), para a Classe $2 \ldots$. . . 88 


\section{Lista de Abreviaturas e Siglas}

$\begin{array}{ll}\text { ACP } & \text { Atendendo o Critério de Parada } \\ \text { CHF } & \text { Custo de Horizonte Finito } \\ \text { CMLP } & \text { Custo Médio a Longo Prazo } \\ \text { EARA } & \text { Equação Algébrica de Riccati Acoplada } \\ \text { EOI } & \text { Estratégia de Observação Indireta } \\ \text { ERR } & \text { Equação Recursiva de Riccati } \\ \text { MN } & \text { Método de Newton } \\ \text { MS } & \text { Mean Square } \\ \text { MV } & \text { Método Variacional } \\ \text { PACP } & \text { Problemas que Atenderam o Critério de Parada } \\ \text { PRNMI } & \text { Problemas que Realizaram o Número Máximo de Iterações } \\ \text { PNR } & \text { Problemas Não Resolvidos } \\ \text { SLSM } & \text { Sistemas Lineares com Saltos Markovianos }\end{array}$





\section{Sumário}

1 Introdução $\quad 15$

1.1 Estrutura da Dissertação . . . . . . . . . . . . . . . . . 16

2 Conceitos e Resultados Preliminares $\quad 19$

2.1 Notações e Definições . . . . . . . . . . . . . . . . . . . . . . . . . . . . 19

2.2 Cadeias de Markov . . . . . . . . . . . . . . . . . . . . . . . . . . . 21

2.3 Sistemas Lineares com Saltos Markovianos . . . . . . . . . . . . . . . . 23

3 Método Variacional $\quad 29$

3.1 Condição Necessária de Otimalidade . . . . . . . . . . . . . . . . . . . 30

3.2 Exemplo Numérico . . . . . . . . . . . . . . . . . . . . . . . . . . . . . 32

4 Método de Direção de Busca 35

4.1 Método de Newton . . . . . . . . . . . . . . . . . . . . . 36

4.2 Cálculo das Derivadas do Custo . . . . . . . . . . . . . . . . . . . 38

4.3 Exemplos Numéricos . . . . . . . . . . . . . . . . . 42

5 Estratégia de Observação Indireta $\quad 47$

5.1 Estratégia de Observação Indireta . . . . . . . . . . . . . . . . . 47

5.2 Método Variacional Adaptado para a Observação Parcial . . . . . . . . 52

5.3 Estratégia de Observação Indireta para o CMLP . . . . . . . . . . . . 55

5.4 Exemplos Numéricos . . . . . . . . . . . . . . . 58

6 Experimentos Computacionais $\quad 63$

6.1 Gerador de SLSM . . . . . . . . . . . . . . . . . . 63

6.2 Resultados Computacionais . . . . . . . . . . . . 66

$\begin{array}{lll}7 & \text { Conclusão } & 91\end{array}$ 
$\begin{array}{ll}\text { Referências Bibliográficas } & 93\end{array}$

$\begin{array}{ll}\text { A Prova dos Teoremas } 4.1 \text { e } 4.2 & 97\end{array}$

B Equações de Riccati 103

$\begin{array}{ll}\text { C Controlabilidade e Observabilidade } & 105\end{array}$

D Sequências de Ganhos Obtidas $\quad 107$ 


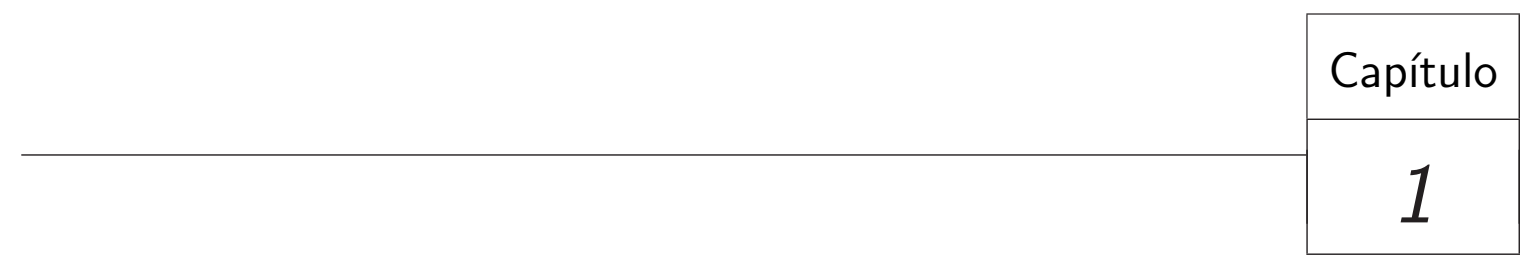

Introdução

Sistemas lineares com saltos markovianos (SLSM) formam uma importante classe de sistemas lineares estocásticos, os quais têm sido foco de pesquisa nos últimos anos. Esse interesse se deve, em grande parte, aos aspectos práticos e aplicações úteis para diversas situações em que há alterações abruptas de comportamento, como por exemplo, vulnerabilidade a falhas de componentes, perturbações ambientais repentinas, entre outras.

Entre os diversos trabalhos que tratam dessas aplicações, destaca-se do Val e Başar (1999), que mostra um estudo de modelos macroeconômicos variantes no tempo, nos quais alguns parâmetros atuam de forma exógena, de acordo com uma cadeia de Markov. Outra importante aplicação é feita em Saridis (1983), que propõe uma abordagem para trabalhar com sistemas robóticos interativos que requerem tomada de decisões avançadas em ambientes imprevisíveis. Uma aplicação financeira é apresentada em Costa e Araújo (2008), no qual é tratado um problema de seleção de carteiras de investimentos multi-período de média-variância com parâmetros de mercado sujeitos a saltos markovianos. Sworder e Rogers (1983) trata do controle de temperatura de sistema de geração de energia através do sol.

Além dos aspectos práticos, o fato de SLSM generalizarem sistemas lineares determinísticos e, ainda, serem suficientemente especializados para apresentar resultados fortes que recuperam propriedades de sistemas lineares clássicos, os torna alvo de vários trabalhos que abordam um estudo mais teórico. Destacam-se os resultados que se referem às Equações Algébricas de Riccati Acopladas (EARA), associadas ao problema Linear Quadrático e ao conceito de MS-estabilizabilidade. Este último garante a exis- 
tência de soluções das EARA. Abordagens desse tema são encontradas em Ji e Chizeck (1990; 1992), Morozan (1995), Costa et al. (1999), Fragoso e Baczynski (2001), entre outros.

Recentemente, a maioria dos estudos têm focado no problema do custo médio a longo prazo (CMLP), que consiste em determinar uma lei de controle tal que minimize o custo associado ao sistema. Em Vargas et al. (2006) e Costa et al. (2011) são mostrados os resultados de uma avaliação que relaciona o custo de horizonte finito (CHF) com o CMLP, associados a um SLSM com ruído aditivo, que permite concluir a existência do CMLP, e de uma solução ótima para o controle.

O CMLP foi bastante estudado em diversos contextos, e em particular para SLSM no cenário com observação. Em Costa et al. (2005) o cálculo do controle ótimo é determinado pela solução de uma EARA, sendo que o controle resulta na forma de realimentação linear de estado.

Para o cenário sem observação, que é frequente em diversas situações práticas, não se conhecem resultados que permitam calcular o controle ótimo. Apenas dispõese de um resultado em que se formula uma expressão para o CMLP, assumindo o controle na forma de realimentação linear (compatível com o caso em que é observado) (VARGAS et al., 2006).

Levando-se em conta que é muito comum não se dispor de medidas exatas e instantâneas do estado da cadeia de Markov (exceto em condições de laboratório), faz-se necessário desenvolver métodos para síntese de controle ótimo (problema de otimização) ou ao menos do CHF (problema de factibilidade). Nesse contexto, há vários aspectos associados que são de interesse para estudo, como a comparação de diferentes métodos para o problema de otimização, condições para convergência e/ou unicidade de solução.

Neste trabalho, é enfatizado o estudo do CHF motivado pelo problema do CMLP. Para isso, são analisados e comparados 3 métodos de otimização: Método Variacional, Método de Direção de Busca e Estratégia de Observação Indireta.

\subsection{Estrutura da Dissertação}

Este trabalho está organizado da seguinte forma: o Capítulo 2 apresenta as notações e definições utilizadas ao longo do texto, bem como a formulação geral do problema estudado. O método variacional desenvolvido por Vargas et al. (2004), e utilizado como parâmetro de comparação entre os métodos desenvolvidos neste trabalho, é apresentado no Capítulo 3. No Capítulo 4 é mostrado o método de direção de busca, o cálculo das derivadas dos custos e exemplos ilustrativos para este método. No Capítulo 5 é proposta 
uma estratégia de observação indireta, sendo que um exemplo numérico é apresentado para ilustrar essa abordagem. No Capítulo 6 é proposto um gerador de SLSM capaz de elaborar um conjunto de diversos exemplos numéricos de SLSM com diferentes características estruturais; além disso, são apresentados os experimentos computacionais realizados para avaliar e comparar o desempenho dos métodos apresentados nos capítulos anteriores. Por fim, no Capítulo 7 são apresentadas as conclusões do trabalho e as propostas para trabalhos futuros. 



\begin{tabular}{|c|c|}
\hline Capítulo \\
\hline 2 \\
\hline
\end{tabular}

\section{Conceitos e Resultados Preliminares}

Este capítulo é organizado da seguinte forma. Na Seção 2.1 são apresentadas as Notações e Definições utilizadas ao longo do texto. Na Seção 2.2 é feita uma breve descrição, baseada em Clarke e Disney (1970), das Cadeias de Markov e de suas principais propriedades. Os Sistemas Lineares com Saltos Markovianos são definidos na Seção 2.3, sendo apresentada uma teoria que culmina na formulação matemática do Problema de Otimização do Custo.

\subsection{Notações e Definições}

Seja $\mathcal{N}=\{1, \ldots, N\}$ um conjunto finito e $\mathcal{M}^{r, s}\left(\mathcal{M}^{r}\right)$ a representação de um espaço linear normado formado por todas as matrizes reais de dimensão $r \times s(r \times r)$. Define-se $\mathbb{M}^{r, s}$ como sendo o espaço linear de todas as $N$-sequências de matrizes tais que $\mathbb{M}^{r, s}=\left\{U=\left(U_{1}, \ldots, U_{N}\right): U_{i} \in \mathcal{M}^{r, s}, i \in \mathcal{N}\right\}$. Seja $\mathcal{S}^{r}$ a representação do subespaço linear normalizado de $\mathcal{M}^{r}$ de matrizes simétricas, ou seja, $\mathcal{S}^{r}=\left\{U \in \mathcal{M}^{r}: U=U^{\prime}\right\}$, onde $U^{\prime}$ denota o transposto de $U$. Considere $\mathcal{S}^{r 0}\left(\mathcal{S}^{r+}\right)$ o cone fechado (aberto) de matrizes semi-definidas (definidas) positivas de $\mathcal{S}^{r}$, ou seja, $\mathcal{S}^{r 0}=\left\{U \in \mathcal{S}^{r}: U \geq 0\right\}$, $\mathcal{S}^{r+}=\left\{U \in \mathcal{S}^{r}: U>0\right\}$. Define-se $\mathbb{S}^{r}$ como sendo o espaço linear de todas as $N$ sequências de matrizes tais que $\mathbb{S}^{r}=\left\{U=\left(U_{1}, \ldots, U_{N}\right): U_{i} \in \mathcal{S}^{r}, i \in \mathcal{N}\right\}$. Além disso, denota-se a matriz identidade de dimensão $r \times r$ por $I^{r}$, o valor esperado por $E\{\cdot\}$ e o operador $\mathbb{1}_{\{F\}}$ representa a medida de Dirac do conjunto $F$. 
Denota-se por $\operatorname{Tr}\{\cdot\}$ o operador traço e define-se a norma

$$
\|U\|_{2}^{2}=\sum_{i=1}^{N} \operatorname{Tr}\left\{U_{i}^{\prime} U_{i}\right\}, \quad \text { para } U \in \mathbb{M}^{r, s} .
$$

O espaço $\mathbb{M}^{r, s}$ equipado com o produto interno, como definido abaixo, forma um espaço de Hilbert.

$$
\langle U, V\rangle=\sum_{i=1}^{N} \operatorname{Tr}\left\{U_{i}^{\prime} V_{i}\right\}
$$

Considere as matrizes $A \in \mathcal{M}^{m, n}$ e $B \in \mathcal{M}^{p, q}$. O Produto de Kronecker de $A$ por $B$, denotado por $A \otimes B$, é dado pela matriz $m p \times n q$

$$
A \otimes B=\left[\begin{array}{ccc}
a_{11} B & \ldots & a_{1 n} B \\
\vdots & \ddots & \vdots \\
a_{m 1} B & \ldots & a_{m n} B
\end{array}\right] .
$$

Define-se por $\operatorname{vec}(A)$ o vetor coluna $m n \times 1$ de todos os elementos da matriz $A$ tomados coluna por coluna, ou seja,

$$
\operatorname{vec}(A)=\left[\begin{array}{llllllll}
a_{11} & a_{21} & \ldots & a_{m 1} & \ldots & a_{1 n} & \ldots & a_{m n}
\end{array}\right]^{\prime} .
$$

Note que vec $(A)$ e vec $\left(A^{\prime}\right)$ contém os mesmos elementos, porém estão em diferente ordem. Define-se $\mathcal{P}_{m, n} \in \mathcal{M}^{m n}$ como a Matriz de Permutação tal que $\operatorname{vec}\left(A^{\prime}\right)=$ $\mathcal{P}_{m, n} \operatorname{vec}(A)$. Pode-se determinar $\mathcal{P}_{m, n}$ da seguinte forma:

$$
\mathcal{P}_{m, n}=\sum_{i=1}^{n}\left(e_{i}^{\prime} \otimes I^{m} \otimes e_{i}\right) \quad \text { ou } \quad \mathcal{P}_{m, n}=\sum_{j=1}^{m}\left(e_{j} \otimes I^{n} \otimes e_{j}^{\prime}\right)
$$

sendo que $e_{i}$ é a $i$-ésima coluna de $I^{n}$ e $e_{j}$ é a $j$-ésima coluna de $I^{m}$.

Propriedades 2.1. (ABADIR; MAGNUS, 2005) Considere as matrizes $A \in \mathcal{M}^{m, n}, B, C \in$ $\mathcal{M}^{p, q}, D \in \mathcal{M}^{n, m}, E \in \mathcal{M}^{q, s}$ e $F \in \mathcal{M}^{n, p}$. Então

a. $(\alpha \otimes A)=\alpha A=A \alpha=(A \otimes \alpha)$, onde $\alpha$ é um escalar;

b. $(A \otimes B)^{\prime}=A^{\prime} \otimes B^{\prime}$;

c. $(A \otimes B)+(A \otimes C)=A \otimes(B+C)$;

d. $(A \otimes B)(D \otimes E)=(A D \otimes B E) ;$

e. $\operatorname{Tr}\{A D\}=\operatorname{vec}\left(A^{\prime}\right)^{\prime} \operatorname{vec}(D)=\operatorname{vec}\left(D^{\prime}\right)^{\prime} \operatorname{vec}(A)$;

f. $\operatorname{vec}(A F B)=\left(B^{\prime} \otimes A\right) \operatorname{vec}(F)$;

g. $\mathcal{P}_{m, n}^{\prime}=\mathcal{P}_{m, n}^{-1}=\mathcal{P}_{n, m}$. 


\subsection{Cadeias de Markov}

Um processo estocástico é uma sequência de variáveis aleatórias, $\theta(k) \in \mathcal{N}$, indexadas por um parâmetro $k$ de um conjunto arbitrário $\mathcal{K}(\mathcal{K} \neq \emptyset)$, sendo representado por $\Theta=\{\theta(k), k \in \mathcal{K}\}$. O conjunto formado pelos valores numéricos que $\theta(k)$ pode assumir é denominado de Estado do Processo; além disso, para $\theta(k)=i$ diz-se que $\theta$ está no estado $i$ no "instante" $k$. O conjunto $\Theta$ é chamado de Espaço de Estados e o conjunto $\mathcal{K}$ é o Espaço de Parâmetros e este está contido no conjunto dos reais.

Um processo estocástico é considerado uma cadeia de Markov quando seu espaço de estados é discreto e possui a propriedade markoviana, ou seja, para cada $k \in \mathcal{K}$ dado um valor $\theta(k)$, os valores de $\theta(k+1)$ não são influenciados pelos valores de $\theta(k-t), t=1, \ldots, k$, e nem de quaisquer outras variáveis, exceto $\theta(k)$.

Seja $P[\theta(k)=i]$ a probabilidade de $\theta(k)$ estar em um determinado estado $i$, no "instante" $k \in \mathcal{K}=\{0,1, \ldots\}$. Então pela propriedade markoviana temos que a probabilidade de $\theta$ estar no estado $j \in \mathcal{N}$ no "instante" $k+1$ sabendo-se que $\theta$ está no estado $i \in \mathcal{N}$ no "instante" $k$ é dada pela probabilidade condicional:

$$
\begin{aligned}
p_{i j}(k) & =P\left[\theta(k+1)=j \mid \theta(0)=i_{0}, \theta(1)=i_{1}, \ldots, \theta(k)=i\right] \\
& =P[\theta(k+1)=j \mid \theta(k)=i]
\end{aligned}
$$

sendo que $i_{0}, i_{1}, \ldots \in \mathcal{N}$.

Observação 2.1. Se a probabilidade de transição não depender do valor de $k$, temos $p_{i j}(k)=p_{i j}$. Neste caso, a cadeia de Markov é chamada de homogênea ou estacionária.

Definição 2.1. A probabilidade de transição de $n$-passos, $p_{i j}^{(n)}$, que representa a probabilidade do processo ir do estado $i$ ao $j$ em n-passos é definida por:

$$
p_{i j}^{(n)}=P[\theta(k+n)=j \mid \theta(k)=i]
$$

para $i, j \in \mathcal{N}, k \in \mathcal{K}$ e para cada $n \geq 1$.

\subsubsection{Classificação de Estados}

1. Um estado $j$ é alcançável do estado $i$ quando se pode atingir $j$ a partir de $i$, em um número finito de transições, isto é se $p_{i j}^{(n)}>0$, para algum $n \geq 1$ e $i, j \in \mathcal{N}$. Além disso, se $p_{j i}^{(m)}>0$ para algum $m$, ou seja, se o estado $i$ á alcançável do estado $j$ então o estado $j$ é comunicante com o estado $i$. Se todos os estados de uma cadeia são comunicantes, então a cadeia é denominada irredutível. 
2. Um estado $i$ é recorrente se $i=j, i, j \in \mathcal{N}$, e $\sum_{s=1}^{\infty} f_{i j}^{(s)}=1$, sendo que $f_{i j}^{(s)}$ é a probabilidade do tempo de primeira passagem do estado $i$ para o estado $j$ em $s$ passos. Caso contrário, o estado é chamado de transiente.

3. Um estado $i$ é periódico de período $d$ se $p_{i i}^{(n)}>0$, somente para $n=d, 2 d, 3 d, \ldots$ onde $d>1$ é o maior inteiro com esta propriedade. Quando todos os estados da cadeia são periódicos, de mesmo período $d$ tem-se uma cadeia periódica de período $d$. Se $d=1$, então $i$ é aperiódico e a cadeia é denominada aperiódica.

4. Se todos os estados de uma cadeia de Markov são comunicantes, recorrentes e aperiódicos entre si, então os estados são denominados de ergódicos e a cadeia é chamada de ergódica.

\subsubsection{Matriz de Transição}

As probabilidades de transição entre estados em um "instante", $p_{i j}$, podem ser representadas por meio de uma matriz $\mathbb{P} \in \mathcal{M}^{N}$ onde $N$ é a quantidade de estados da cadeia, sendo que cada linha $i \in \mathcal{N}=\{1, \ldots, N\}$ representa o estado atual e cada coluna $j \in \mathcal{N}$ representa o estado futuro. Além disso, tem a condição $\sum_{i=1}^{N} p_{i j}=1, j \in \mathcal{N}$.

$$
\mathbb{P}=\left[\begin{array}{cccc}
p_{11} & p_{12} & \ldots & p_{1 N} \\
p_{21} & p_{22} & \ldots & p_{2 N} \\
\vdots & \vdots & \vdots & \vdots \\
p_{N 2} & p_{N 2} & \ldots & p_{N N}
\end{array}\right]
$$

\section{Definição 2.2.}

- A probabilidade inicial da variável aleatória $\theta$ estar no estado $i \in \mathcal{N}$ é dada por $\pi_{i}(0)=P[\theta(0)=i]$. O vetor $\pi(0)=\left[\pi_{1}(0) \ldots \pi_{N}(0)\right]$ é denominado vetor de distribuição de probabilidade inicial.

- O vetor $\pi(n)=\left[\pi_{1}(n) \ldots \pi_{N}(n)\right] \operatorname{com} \pi_{i}(n)=P[\theta(n)=i], i \in \mathcal{N}$, é denominado vetor de distribuição de probabilidades após $n$-passos e pode ser determinado pelo vetor de condições iniciais $\pi(0)$ e pela n-ésima potência da matriz $\mathbb{P}$, isto é, $\pi(n)=\pi(0) \mathbb{P}^{(n)}$.

- Quando existir, a probabilidade de regime permanente para o estado $i \in \mathcal{N}$ é dada por

$$
\bar{\pi}_{i}=\lim _{n \rightarrow \infty} \pi_{i}(n)
$$


Teorema 2.1. (CLARKE; DISNEY, 1970) Em uma cadeia ergódica os limites $\bar{\pi}_{i}=$ $\lim _{n \rightarrow \infty} \pi_{i}(n), i \in \mathcal{N}$, sempre existem e são independentes do vetor de probabilidade inicial.

\subsection{Sistemas Lineares com Saltos Markovianos}

Seja $(\Omega, \mathcal{F}, P)$ o espaço de probabilidade fundamental. Considere $\Theta=\{\theta(k), k \in$ $\mathcal{K}=0,1, \ldots\}$ uma cadeia de Markov homogênea tal que a matriz de transição é denotada por $\mathbb{P}=\left[p_{i j}\right], i, j \in \mathcal{N}$.

Considere o sistema linear com saltos markovianos

$$
\Phi: \begin{cases}x_{k+1}=A_{\theta(k)} x_{k}+B_{\theta(k)} u_{k}+G_{\theta(k)} w_{k}, & \forall k \geq 0, \\ y_{k}=x_{k}^{\prime} C_{\theta(k)} x_{k}+u_{k}^{\prime} D_{\theta(k)} u_{k}, & \theta(0) \sim \pi(0),\end{cases}
$$

onde o par $(x, \theta)$ forma o estado do sistema, $y_{k}$ consiste em um índice de desempenho associado ao estágio $k$, chamado de custo por estágio, e $u_{k}$ é o controle, uma variável cujo valor podemos definir ou projetar de forma a minimizar um critério associado ao custo por estágio. Neste trabalho, o processo estocástico $\left\{w_{k}, k \geq 0\right\}$ é uma sequência de variáveis aleatórias independente e identicamente distribuída (iid) com média zero e covariância igual a matriz identidade para cada $k \in \mathcal{K}$. Além disso, o estado da cadeia de Markov, $\theta(k)$, é parcialmente observado ou é não observado.

A cada "instante" $k$, dado $\theta(k)=i$, tem-se $A_{\theta(k)}=A_{i}$ e similarmente para as demais matrizes da Equação 2.11, sendo que $A_{i} \in \mathcal{M}^{r}, B_{i} \in \mathcal{M}^{r, s}, G_{i} \in \mathcal{M}^{r, l}, C_{i} \in \mathcal{S}^{r 0}$ e $D_{i} \in \mathcal{S}^{s+}$ (veja o Exemplo 2.1).

Exemplo 2.1. (Adaptado de Costa e do Val (1998)) Considere um sistema de produção de uma indústria que fabrica um único produto. A demanda por este produto será representada por uma sequência de variáveis aleatórias $\left\{w_{k}, k \geq 0\right\}$ iid com média $E\left\{w_{k}\right\}=w_{0}>0$. A indústria deseja que a sua produção satisfaça toda a demanda. No entanto, deve-se considerar que o sistema de produção está sujeito a falhas, e portanto, podemos ter dois possíveis estados de Markov: sistema de produção operando (estado 1) ou não operando (estado 0). Pode-se representar este processo pelo seguinte diagrama (Figura 2.1), onde estão representadas as probabilidades de transição de cada estado: 


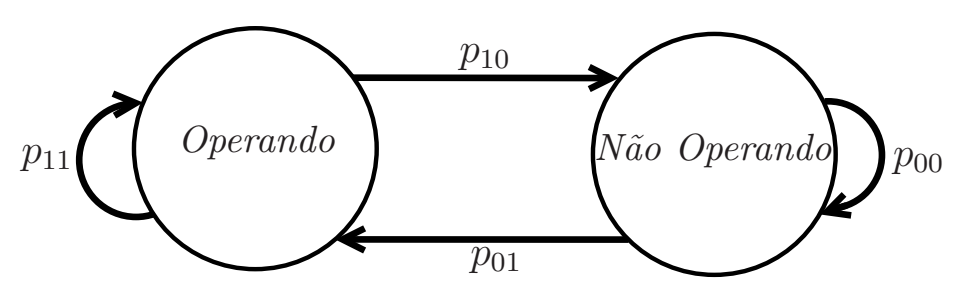

Figura 2.1: Diagrama de estados

Sejam $x_{k}, u_{k}$ e $w_{k}$, o estoque do produto, a produção total e a demanda no tempo $k$, respectivamente. Dessa forma, temos que o estoque no tempo $k+1$ é dado por:

$$
x_{k+1}=A_{\theta(k)} x_{k}+B_{\theta(k)} u_{k}-G_{\theta(k)} w_{k},
$$

com $A_{0}=A_{1}=B_{1}=G_{0}=G_{1}=1$ e $B_{0}=0$. A matriz de transição desse processo é denotada por $\mathbb{P}=\left[p_{i j}\right], i, j=0,1$, com $p_{i j} \in(0,1)$. O problema consiste em controlar a produção $u_{k}$ no tempo $k$ de modo a minimizar

$$
\sum_{k=0}^{\infty} E_{\left\{\theta(0), x_{0}\right\}}\left\{C_{\theta(k)} x_{k}^{2}+D_{\theta(k)} u_{k}^{2}\right\},
$$

sendo que $C_{0}=C_{1}=\rho^{k} m$ e $D_{0}=D_{1}=\rho^{k} \operatorname{com} m>0$ e $\rho \in(0,1)$.

Definição 2.3. Considere as matrizes $\mathbb{P}=\left[p_{i j}\right] \in \mathcal{M}^{N}, G \in \mathbb{M}^{r, l}, V \in \mathbb{M}^{r}$ e $U, \psi \in \mathbb{S}^{r 0}$. Definem-se para todo $i \in \mathcal{N}$ e $k \in \mathcal{K}$ os operadores lineares $\mathscr{E}, \mathcal{L}_{V}, \mathcal{T}_{V}: \mathbb{S}^{r 0} \rightarrow \mathbb{S}^{r 0}$ e a sequência de matrizes $\Sigma(k) \in \mathbb{S}^{r 0}$ tais que:

$$
\begin{gathered}
\mathscr{E}_{i}(\psi)=\sum_{j=1}^{N} p_{i j} \psi_{j}, \\
\mathcal{L}_{V, i}(U)=V_{i}^{\prime} \mathscr{E}_{i}(U) V_{i}, \\
\Sigma_{i}(k)=\sum_{j=1}^{N} p_{j i} \pi_{j}(k) G_{j} G_{j}^{\prime}, \\
\mathcal{T}_{V, i}(U)=\sum_{j=1}^{N} p_{j i} V_{j} U_{j} V_{j}^{\prime} .
\end{gathered}
$$

Para o operador $\mathcal{T}_{V}$, define-se $\mathcal{T}_{V}^{0}(U)=U$ e para $t \geq 1$ tem-se a recursão $\mathcal{T}_{V}^{(t)}(U)=$ $\mathcal{T}_{V}\left(\mathcal{T}_{V}^{(t-1)}(U)\right)$. 
Definição 2.4. O segundo momento de $x_{k}$, condicionado ao estado markoviano $\theta(k)$ é definido por $X(k)=\left(X_{1}(k), \ldots, X_{N}(k)\right) \in \mathbb{S}^{r 0}$ com

$$
X_{i}(k)=E\left\{x_{k} x_{k}^{\prime} \mathbb{1}_{\{\theta(k)=i\}}\right\}, \quad \forall i \in \mathcal{N} \text { e } k \in \mathcal{K} .
$$

Lema 2.1. (VARGAS, 2004, Lema 2.1) Dada uma sequência de matrizes $U=\left(U_{i}, i \in\right.$ $\mathcal{N}) \in \mathbb{S}^{r 0}$, vale a identidade

$$
E\left\{x_{k}^{\prime} U_{\theta(k)} x_{k}\right\}=\sum_{i=1}^{N} \operatorname{Tr}\left\{U_{i} X_{i}(k)\right\}=\langle X(k), U\rangle, \quad \forall k \in \mathcal{K} .
$$

\subsubsection{Formulação do Problema de Otimização do Custo}

Considere o SLSM $\Phi$ (Equação 2.11). Assumimos uma lei de controle linear na forma

$$
u_{k}=g(k) x_{k} \quad \operatorname{com} \quad k \geq 0,
$$

sendo que a matriz $g(k) \in \mathcal{M}^{s, r}$ é denominada de ganho. Denota-se por $\mathcal{G}$ o conjunto formado por todas as possíveis sequências de ganhos $\mathbf{g}=\{g(0), g(1), \ldots\} \in \mathcal{G}$. Substituindo a lei de controle dada pela Equação 2.20 em $\boldsymbol{\Phi}$, obtém-se o seguinte SLSM:

$$
\overline{\mathbf{\Phi}}: \begin{cases}x_{k+1}=\bar{A}_{\theta(k)} x_{k}+G_{\theta(k)} w_{k}, & \forall k \geq 0, \\ y_{k}=x_{k}^{\prime} \bar{C}_{\theta(k)} x_{k}, & \theta(0) \sim \pi(0),\end{cases}
$$

$\operatorname{com} \bar{A}_{\theta(k)}=A_{\theta(k)}+B_{\theta(k)} g(k) \in \mathcal{M}^{r}$ e $\bar{C}_{\theta(k)}=C_{\theta(k)}+g(k)^{\prime} D_{\theta(k)} g(k) \in \mathcal{S}^{r+}$.

A Proposição 2.1 apresenta uma expressão determinística que facilita o cálculo de $X(k)$ (Definição 2.4).

Proposição 2.1. (COSTA et al., 2005, Ch. 3) Considere $V(k) \in \mathbb{S}^{r 0}$ definido de acordo com

$$
\left\{\begin{array}{l}
V(k+1)=\mathcal{T}_{\bar{A}}(V(k))+\Sigma(k), \quad k \geq 0 \\
V_{i}(0)=V \in \mathcal{S}^{r 0}
\end{array}\right.
$$

Fazendo $V(k)=X(k)$ com a condição inicial $V_{i}(0)=\pi_{i}(0) x_{0} x_{0}^{\prime}$, para todo $i \in \mathcal{N}$, obtém-se uma expressão para determinar $X(k)$ (Definição 2.4), ou seja,

$$
X(k+1)=\mathcal{T}_{\bar{A}}(X(k))+\Sigma(k), \quad k \geq 0 .
$$


Exemplo 2.2. (Adaptado de Manfrim (2006)) Considere os seguintes parâmetros para o SLSM $\boldsymbol{\Phi}$ (Equação 2.11),

$$
\begin{gathered}
\bar{A}_{1}=\left[\begin{array}{ccc}
0,9 & 0,1 & 0,1 \\
0 & 1,1 & 0 \\
0 & 0 & 0,9
\end{array}\right], \bar{A}_{2}=\left[\begin{array}{ccc}
0,9 & 0,1 & 0 \\
0,1 & 0,9 & 1 \\
0 & 0 & 0,9
\end{array}\right], \\
G_{1}=\left[\begin{array}{lll}
0 & 0 & 0 \\
0 & 0 & 0 \\
0 & 0 & 1
\end{array}\right], G_{2}=\left[\begin{array}{lll}
0 & 0 & 0 \\
0 & 0 & 0 \\
0 & 0 & 0
\end{array}\right], \mathbb{P}=\left[\begin{array}{ll}
0.1 & 0.9 \\
0.1 & 0.9
\end{array}\right]
\end{gathered}
$$

e $\bar{C}_{1}=\bar{C}_{2}=I^{3} \operatorname{com} w_{k} \sim N\left[0, Q=C_{k} C_{k}^{\prime}\right]$ e $x_{0} \sim N[\mu, \epsilon]$ onde $\mu, \epsilon$ denotam a média e a covariância de $x_{0}$, respectivamente. Adotam-se as seguintes medidas para o sistema: $\mu^{(1)}=\left[\begin{array}{lll}0 & 0 & 0\end{array}\right]^{T}$ e $\mu^{(2)}=\left[\begin{array}{lll}90 & 90 & 90\end{array}\right]^{T} e \epsilon^{(1)}=\epsilon^{(2)}=0$.

Na Figura 2.2 estão ilustradas 50 realizações de $x_{k}^{\prime} x_{k}$ (uma para cada realização de $\theta$ ), o valor esperado $E\left\{x_{k}^{\prime} x_{k}\right\}=\sum_{i=1}^{N} X_{i}(k)$ e a média das realizações de $x_{k}^{\prime} x_{k}$, sugerindo que estes dois últimos valores praticamente coincidem, confirmando a Proposição 2.1.

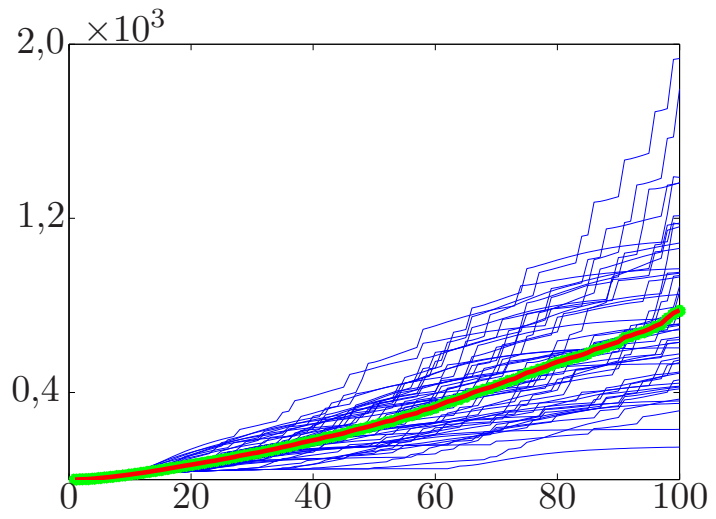

(a) Curvas para $\mu^{(1)}$ e $\epsilon^{(1)}$

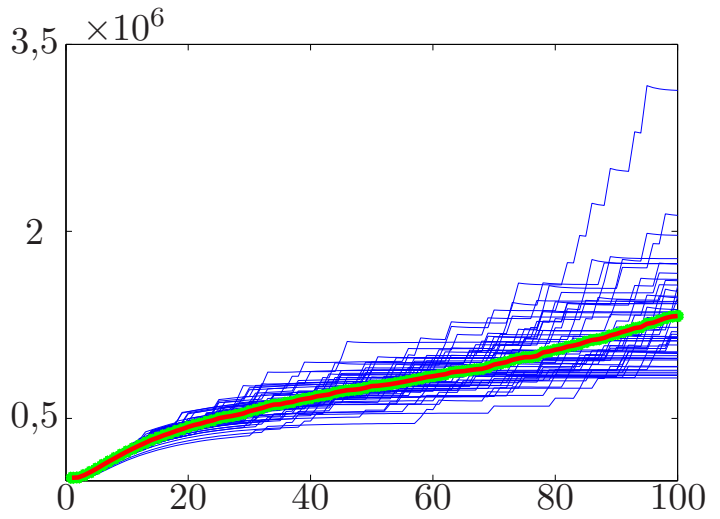

(b) Curvas para $\mu^{(2)}$ e $\epsilon^{(2)}$

Figura 2.2: Realizações de $x_{k}^{\prime} x_{k}$ (em azul), sua média (em vermelho) e o valor esperado $E\left\{x_{k}^{\prime} x_{k}\right\}=\sum_{i=1}^{n} X_{i}(k)$ (em verde), referentes ao Exemplo 2.2

Pela Proposição 2.1 pode-se definir o Custo por Estágio

$$
J_{k}=E\left\{y_{k}\right\}=E\left\{x_{k}^{\prime} \bar{C}_{\theta(k)} x_{k}\right\}=\langle X(k), \bar{C}\rangle, \forall k \in \mathcal{K} .
$$

Além disso, pode-se definir o Custo de T Estágios para o sistema $\overline{\mathbf{\Phi}}$ (Equação 2.21) da seguinte forma:

$$
\mathcal{J}_{T}=\sum_{k=0}^{T-1} E\left\{y_{k}\right\}=\sum_{k=0}^{T-1} J_{k} .
$$


A partir destes resultados, pode-se introduzir o problema de otimização, que consiste em determinar uma sequência de ganhos $\mathrm{g} \in \mathcal{G}$, que minimize o índice de desempenho em termos do valor esperado. Formalmente, tem-se o seguinte problema

$$
\begin{aligned}
& \min _{\mathbf{g} \in \mathcal{G}} \mathcal{J}_{T}=\sum_{k=0}^{T-1} J_{k} \\
& \text { s.a. }\left\{\begin{array}{l}
X_{i}(k+1)=\mathcal{T}_{\bar{A}, i}(X(k))+\Sigma_{i}(k), \quad k \geq 0, \\
X_{i}(0)=\pi_{i}(0) x_{0} x_{0}^{\prime}, \quad i \in \mathcal{N}=\{1, \ldots, N\} .
\end{array}\right.
\end{aligned}
$$

Neste trabalho, será considerado o caso em que $N \geq 2$, pois para $N=1 \mathrm{o}$ problema se reduz ao caso sem saltos nos parâmetros, que já é bem conhecido e tem solução analítica via equações de Riccati. Além disso, apesar de ser enfatizado o estudo do Problema 2.26. A seguir, é apresentada a formulação do problema referente ao Custo Médio a Longo Prazo, o qual é o foco de vários estudos presente na literatura, como exemplo, em Costa et al. (2005), Vargas et al. (2006) e Costa et al. (2011).

Considere o CMLP referente ao SLSM $\overline{\boldsymbol{\Phi}}$ (Equação 2.21) dado por

$$
J=\limsup _{T \rightarrow \infty} \frac{\partial_{T}}{T}
$$

Hipótese 2.1. A cadeia de Markov $\Theta=\{\theta(k), k \in \mathcal{K}\}$ é ergódica.

Observação 2.2. A Hipótese 2.1 é adotada simplesmente para facilitar a notação, pois o caso geral pode envolver, por exemplo, limites periódicos na Proposição 2.2 (veja COSTA et al., 2011).

Definição 2.5. Diz-se que $(A, \mathbb{P})$ é estável na média quadrática (MS-estável, do inglês, Mean Square Stable) se, para cada $x_{0}$ e $\pi(0)$

$$
\lim _{k \rightarrow \infty} E\left\{\left\|z_{k}\right\|^{2}\right\}=0, \quad \forall x_{0}, \pi(0),
$$

sendo $z_{k+1}=A_{\theta(k)} z_{k} \operatorname{com} z_{0} \sim x_{0}$ e $\theta(0) \sim \pi(0)$.

Definição 2.6. Considere o sistema $\mathbf{\Phi}$ (Equação 2.11). Diz-se que $(A, B, \mathbb{P})$ é estabilizável na média quadrática (MS-estabilizável) se existe um conjunto de matrizes $g \in \mathbb{M}^{s, r}$ tal que $(\bar{A}=A+B g, \mathbb{P})$ é $M S$-estável. Neste caso, diz-se que g estabiliza $(A, B, \mathbb{P})$.

Proposição 2.2. (COSTA et al., 2005, Prop. 3.36) Suponha que $(\bar{A}, \mathbb{P})$ é MS-estável. Então existe um único $X \in \mathcal{S}^{r 0}$ que satisfaz $X=\mathcal{T}_{\bar{A}}(X)+\Sigma$, ou equivalentemente

$$
X=\lim _{k \rightarrow \infty} X(k) \quad \text { e } \Sigma=\lim _{k \rightarrow \infty} \Sigma(k) .
$$

Além disso, tem-se que $X=\sum_{k=0}^{\infty} \mathcal{T}_{\bar{A}}^{(k)}(\Sigma)$. 
Corolário 2.1. (VARGAS et al., 2006, Corol. 1) Suponha que $\Theta$ é ergódica e seja g um ganho que estabiliza $(A, B, \mathbb{P})$. Então,

$$
J=\langle X, \bar{C}\rangle,
$$

sendo que $X=\sum_{k=0}^{\infty} \mathcal{T}_{\bar{A}}^{(k)}(\Sigma)$ e $\bar{C}_{i}=C_{i}+g^{\prime} D_{i} g \in \mathbb{S}^{r+}$ para todo $i \in \mathcal{N}$.

Logo, pelos resultados obtidos, pode-se definir o problema de otimização do CMLP que consiste em determinar $g \in \mathcal{M}^{s, r}$ que minimize $J$ (Equação 2.30), matematicamente tem-se que

$$
\begin{gathered}
\min _{g \in \mathcal{M}^{s, r}} J=\langle X, \bar{C}\rangle \\
\text { s.a. }\left\{\begin{array}{l}
X=\mathcal{T}_{\bar{A}}(X)+\Sigma, \\
\mathcal{T}_{\bar{A}}(I)-I<0,
\end{array}\right.
\end{gathered}
$$

sendo que a segunda restrição em (2.31) refere-se a garantia de MS-estabilidade de $\bar{A}$.

Observação 2.3. Os resultados acima são facilmente adaptados para o caso em que se observa a cadeia de Markov $\theta$ e em que $u_{k}=g_{\theta(k)} x_{k}$.

O problema apresentado na Equação 2.26 ainda permanece em aberto na literatura, pois não existe um método de otimização que determine uma sequência de ganhos ótimos, g* $^{*}$ tal que o custo de $T$ estágios seja um mínimo global. Analogamente, o problema da Equação 2.31 também permance em aberto devido a não existir um método que determine a solução ótima $g^{*}$.

No entanto, há um algoritmo baseado no método variacional, proposto em do Val e Başar (1999) e também estudado em Vargas et al. (2004), para o problema do custo de T estágios (que pode ser utilizado para aproximar o problema do CMLP, vide Costa et al. (2011)). Este algoritmo é baseado em uma condição necessária de otimalidade, portanto encontra um mínimo local de $\mathcal{J}_{T}$. 


\begin{tabular}{l|c|}
\cline { 2 - 2 } & Capítulo \\
\cline { 2 - 2 } & 3 \\
\hline
\end{tabular}

Método Variacional

O método variacional é utilizado em várias áreas da ciência, tais como física (cálculo variacional), estatística (problema de momentos linear e não-linear), engenharia (teoria de controle), entre outras. Este método consiste em minimizar(maximizar) uma função por meio de pequenas variações em alguns de seus parâmetros, utilizando o princípio variacional (veja Kirk (2004) e/ou Baumeister e Leitão (2008) para maiores detalhes).

Em 1999, do Val e Başar desenvolveram um algoritmo baseado no princípio variacional para problemas relacionados aos SLSM sem ruído. Posteriormente, tal algoritmo foi estendido para os problemas com ruído aditivo por Vargas et al. (2004) e para sistemas com ruído multiplicativo por Furloni (2009).

Neste capítulo, são apresentados alguns detalhes do algoritmo desenvolvido por Vargas et al. (2004) a fim de utilizá-lo como parâmetro de comparação para avaliar os métodos de otimização desenvolvidos para o problema do custo de T estágios (Equação 2.26).

Este capítulo é organizado da seguinte forma. Na Seção 3.1 é apresentada a Condição Necessária de Otimalidade, baseado fortemente em Vargas (2009), que resulta em uma sequência de ganhos $\mathrm{g} \in \mathcal{G}$ para o custo de $\mathrm{T}$ estágios, $\mathcal{J}_{T}$. Um Exemplo Numérico ilustra a utilização deste método na Seção 3.2. 


\subsection{Condição Necessária de Otimalidade}

Considere o problema formulado na Equação 2.26

$$
\begin{aligned}
& \min _{\mathbf{g} \in \mathcal{G}} \mathcal{J}_{T}=\sum_{k=0}^{T-1}\langle X(k), \bar{C}\rangle \\
& \text { s.a. }\left\{\begin{array}{l}
X_{i}(k+1)=\mathcal{T}_{\bar{A}, i}(X(k))+\Sigma_{i}(k), \quad k \geq 0, \\
X_{i}(0)=\pi_{i}(0) x_{0} x_{0}^{\prime}, \quad i \in \mathcal{N} .
\end{array}\right.
\end{aligned}
$$

sendo que $\bar{C}_{i}=C_{i}+g(k)^{\prime} D_{i} g(k) \in \mathbb{S}^{r+}$, para todo $i \in \mathcal{N}$, a cadeia de Markov, $\theta$, não é observada e os operadores $\mathcal{T}_{\bar{A}}$ e $\Sigma$ são dados pela Definição 2.3. Os resultados a seguir apresentam uma condição de otimalidade para este problema.

Definição 3.1. Considere os operadores $\mathscr{E}, \mathcal{L}: \mathbb{S}^{r 0} \rightarrow \mathbb{S}^{r 0}$ como na Definição 2.3. Então, definem-se $L(k) \in \mathbb{S}^{r 0}$ e $\omega(k) \in \mathbb{M}^{1}$, para cada $k=0,1, \ldots, T-1$, tais que:

$$
\begin{array}{r}
L_{i}(k)=C_{i}+g(k)^{\prime} D_{i} g(k)+\mathcal{L}_{\bar{A}}(L(k+1)), \\
\omega_{i}(k)=\mathscr{E}(\omega(k+1))+\operatorname{Tr}\left\{\mathscr{E}(L(k+1)) G_{i} G_{i}^{\prime}\right\},
\end{array}
$$

sendo que $L_{i}(T)=0$ e $\omega_{i}(T)=0$, para todo $i \in \mathcal{N}$.

Proposição 3.1. (VARGAS et al., 2004, Prop. 3.1; VARGAS, 2009, Prop. 5.3) Para cada $k_{0}=\{0,1, \ldots, T-1\}$ vale a identidade

$$
\begin{aligned}
\sum_{k=k_{0}}^{T-1} E\left\{y_{k}\right\} & =\sum_{k=k_{0}}^{T-1} E\left\{x_{k}^{\prime}\left(C_{\theta(k)}+g(k)^{\prime} D_{\theta(k)} g(k)\right) x_{k}\right\} \\
& =\left\langle L\left(k_{0}\right), X\left(k_{0}\right)\right\rangle+\pi\left(k_{0}\right)^{\prime} \omega\left(k_{0}\right) .
\end{aligned}
$$

O Teorema 3.1 apresenta uma condição de otimalidade sob o ganho $g(k) \in \mathcal{M}^{s, r}$. A sua demonstração é obtida utilizando a Proposição 3.1 com um argumento de programação dinâmica (BERTSEKAS, 1987) e pode ser encontrada em Vargas et al. (2004).

Teorema 3.1. (VARGAS et al., 2004, Theo. 3.1; VARGAS, 2009, Teo. 5.3) Suponha que a sequência de ganhos $\mathbf{g} \in \mathcal{G}$ realiza o minimo global do problema do custo de $T$ estágios. Então g satisfaz, para cada $k \in \mathcal{K}$, a equação

$$
\sum_{i=1}^{N}\left[\left(D_{i}+B_{i}^{\prime} \mathscr{E}_{i}(L(k+1)) B_{i}\right) g(k)+B_{i}^{\prime} \mathscr{E}_{i}(L(k+1)) A_{i}\right] X_{i}(k)=0 .
$$

Observação 3.1. Um método para obter a solução da equação algébrica 3.5 pode ser encontrado em do Val e Başar (1999) e Vargas (2004). 
No Algoritmo 3.1 é apresentado o método variacional que resulta em uma sequência de ganhos $\mathbf{g}=\{g(0), \ldots, g(T-1)\}$ que minimiza localmente o custo por $T$ estágios, $\mathcal{J}_{T}$, satisfazendo a condição de otimalidade (Equação 3.5).

Algoritmo 3.1 Método Variacional

Passo 0: Inicie o processo em $\eta=0$.

Escolha uma sequência inicial de ganhos $\mathbf{g}^{(\eta)}=\left\{g^{(\eta)}(0), \ldots, g^{(\eta)}(T-1)\right\}$.

Passo 1: Para todo $k \in \mathcal{K}$ encontre $X^{(\eta)}(k) \in \mathcal{S}^{r 0}$ tal que

$$
\left\{\begin{array}{l}
X^{(\eta)}(k+1)=\mathcal{T}_{\bar{A}}\left(X^{(\eta)}(k)\right)+\Sigma(k), \\
X_{i}^{(\eta)}(0)=\pi_{i}(0) x_{0} x_{0}^{\prime}, \forall i \in \mathcal{N},
\end{array}\right.
$$

onde os operadores $\mathcal{T}$ e $\Sigma$ são dados pela Definição 2.3 .

Passo 2: Faça $\eta=\eta+1$.

Para cada $k=T-1, T-2, \ldots, 0$, determine $g^{(\eta)}(k)$ resolvendo a Equação 3.5 e calcule $L^{(\eta)} \in \mathcal{S}^{r 0}$ pela Equação 3.2.

Critério de Parada: $\left|\mathcal{J}_{T}\left(\mathbf{g}^{(\eta)}\right)-\mathcal{J}_{T}\left(\mathbf{g}^{(\eta-1)}\right)\right| / \mathcal{J}_{T}\left(\mathbf{g}^{(\eta)}\right)<\epsilon$, para $\epsilon$ dado. Se o critério não for satisfeito, volte para o passo 2.

Pelo Teorema 3.2 tem-se a garantia que a sequência de ganhos $\mathbf{g} \in \mathcal{G}$ satisfaz a restrição do Problema 3.1 e as Equações 3.2 e 3.5, logo g é um mínimo local para o problema do custo de $T$ estágios, porém a caracterização desta solução em ser um mínimo local ou global ainda encontra-se em discussão na literatura.

No entanto, de acordo com Vargas (2009), há um forte indício numérico de que o custo obtido pelo Algoritmo 3.1 seja um mínimo global, pois o método sempre converge para um custo mínimo independente da sequência inicial de ganhos, $\mathbf{g}^{(0)}$. De fato, para os exemplos considerados em Vargas (2009), o resultado obtido parece ser o mínimo global, mas este resultado ainda não foi generalizado para todos os SLSM.

Teorema 3.2. (VARGAS et al., 2004, Theo. 3.2) As sequências $\mathbf{g}^{(\eta)}, \eta=0,1,2, \ldots$, geradas no Algoritmo 3.1 são tais que

$$
\partial_{T}\left(\mathbf{g}^{(\eta+1)}\right) \leq \mathcal{J}_{T}\left(\mathbf{g}^{(\eta)}\right) \quad e \quad \mathbf{g}=\lim _{\eta \rightarrow \infty} \mathbf{g}^{(\eta)}
$$

satisfaz o Teorema 3.1.

Observação 3.2. Considerando os indícios observados, Vargas (2009) introduziu a Conjectura 3.1. Supondo que a mesma seja válida, então pode-se determinar o CMLP (Equação 2.31), J*, por meio da seguinte aproximação: $J_{T}^{*} / T \rightarrow J^{*}$ quando $T \rightarrow \infty$ (veja Vargas (2009) para maiores detalhes). 
Conjectura 3.1. (VARGAS, 2009, Conj. 5.1) Seja $\mathbf{g}^{(\eta)}, \eta=0,1,2, \ldots$, gerado no Algoritmo 3.1. Então

$$
\mathcal{J}_{T}^{*}=\mathcal{J}_{T}(\mathbf{g})=\lim _{\eta \rightarrow \infty} \mathcal{J}_{T}\left(\mathbf{g}^{(\eta)}\right)
$$

no qual $\mathbf{g}=\lim _{\eta \rightarrow \infty} \mathbf{g}^{(\eta)}$.

\subsection{Exemplo Numérico}

Nesta seção é apresentado um exemplo numérico para ilustrar a utilização do método variacional cujo resultado pode ser uma aproximação para a solução do problema do custo de T estágios (Equação 3.1).

Exemplo 3.1. Considere os seguintes parâmetros para o SLSM $\Phi$ (Equação 2.11),

$$
\begin{aligned}
& A_{1}=\left[\begin{array}{rrr}
0,7633 & -0,0215 & 0,1252 \\
-0,6456 & 0,2100 & -0,0592 \\
-0,4566 & -0,2645 & 0,5546
\end{array}\right], A_{2}=\left[\begin{array}{rrr}
-6,7366 & 7,5238 & -1,0969 \\
-5,2434 & 5,7396 & -0,6748 \\
2,5139 & -3,4616 & 1,0579
\end{array}\right], \\
& C_{1}=\left[\begin{array}{rrr}
1,2178 & 0,4220 & -0,3873 \\
0,4220 & 0,1463 & -0,1342 \\
-0,3873 & -0,1342 & 0,1231
\end{array}\right], C_{2}=\left[\begin{array}{rrr}
18,5930 & -21,9334 & 6,0864 \\
-21,9334 & 26,1372 & -7,3977 \\
6,0864 & -7,3977 & 2,1727
\end{array}\right] \text {, } \\
& B_{1}=\left[\begin{array}{l}
0,4313 \\
0,0739 \\
3,9401
\end{array}\right], B_{2}=\left[\begin{array}{r}
-0,9231 \\
-0,7560 \\
0,3353
\end{array}\right], G_{1}=\left[\begin{array}{r}
-0,2889 \\
1,9192 \\
4,6867
\end{array}\right], G_{2}=\left[\begin{array}{r}
-0,0773 \\
-0,0126 \\
0,4450
\end{array}\right] \text {, } \\
& \mathbb{P}=\left[\begin{array}{ll}
0,7514 & 0,2486 \\
0,0178 & 0,9822
\end{array}\right], \pi(0)=\left[\begin{array}{ll}
0,3263 & 0,6737
\end{array}\right], x_{0}=\left[\begin{array}{r}
-1,1634 \\
1,1837 \\
-0,0154
\end{array}\right],
\end{aligned}
$$

$D_{1}=0,15$ e $D_{2}=0,5861$.

O Algoritmo 3.1 foi implementado no software $M A T L A B^{\circledR}$ com tolerância $\epsilon=$ $10^{-4}$, horizonte $T=100$ e foi iniciado com a sequência de ganhos $\mathbf{g}^{(0)}$ tal que $g^{(0)}(k)=$ $\left[\begin{array}{lll}0 & 0 & 0\end{array}\right]$, para todo $k \in \mathcal{K}$. O algoritmo realizou $\eta=5$ iterações, interrompendo o processo com o critério de parada igual a aproximadamente $6,0739 \times 10^{-5}$. O custo de T estágios obtido foi igual a

$$
\mathcal{J}_{T}\left(\mathbf{g}^{*}\right) \approx 546,2521
$$

sendo que a sequência de ganhos g* obtida está disponível no Apêndice D.

Na Figura 3.1 são apresentados os comportamentos da norma de 9 ganhos $\|g(k)\|, k \in$ $\mathcal{K}$, da norma do segundo momento $X(T)$ e do custo de $T$ estágios $\mathcal{J}_{T}$, em função do número de iterações $\eta$. Note que que a sequência de pontos em todos os gráficos convergem conforme o número de iterações, $\eta$, aumenta. 


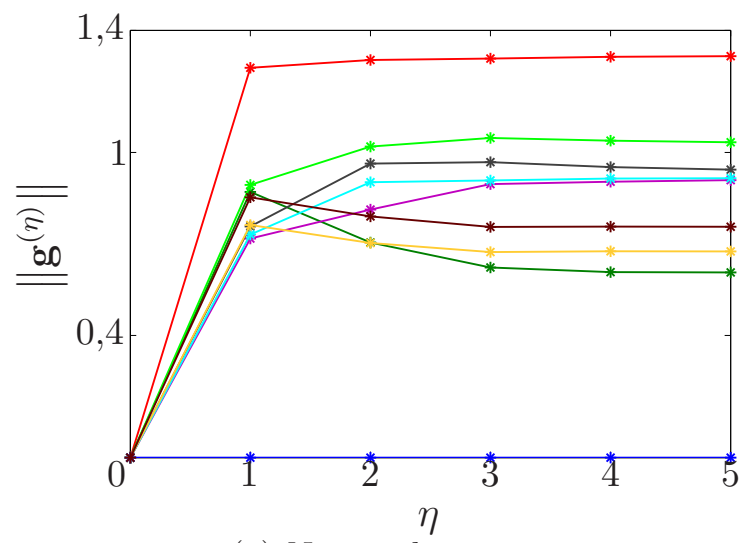

(a) Norma de g versus $\eta$

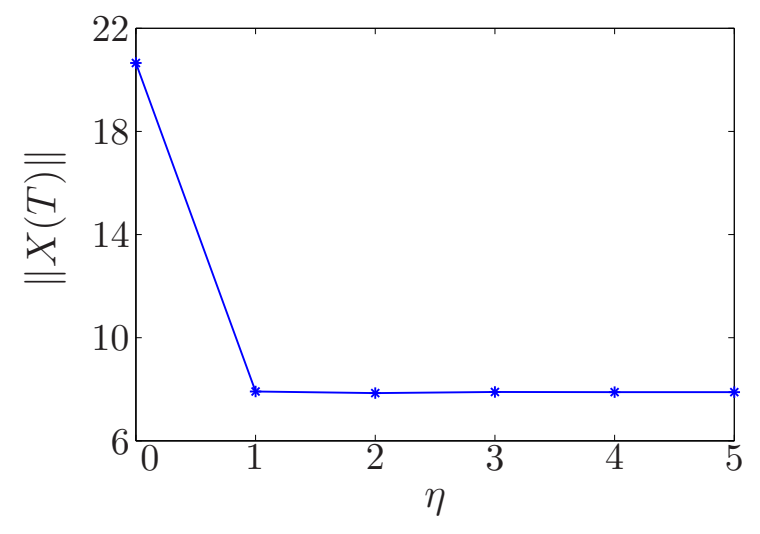

(b) Norma de $X(T)$ versus $\eta$

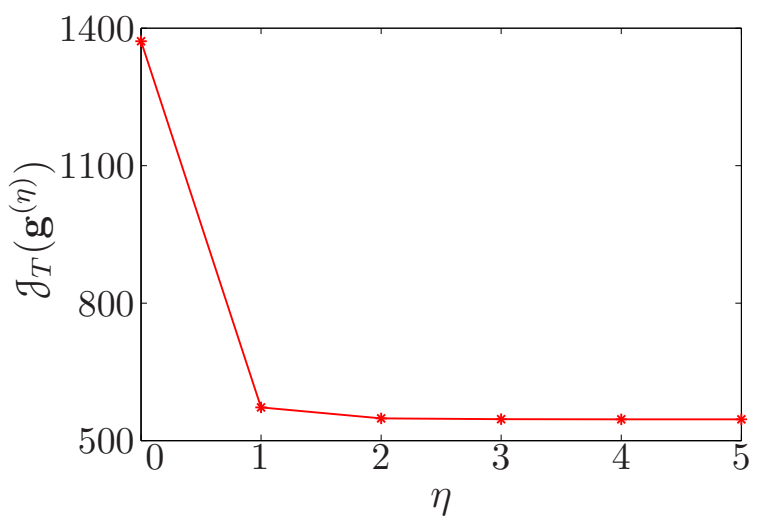

(c) Custo $\mathcal{J}_{T}\left(\mathbf{g}^{(\eta)}\right)$ versus $\eta$

Figura 3.1: Resultados do método variacional para o Exemplo 3.1 



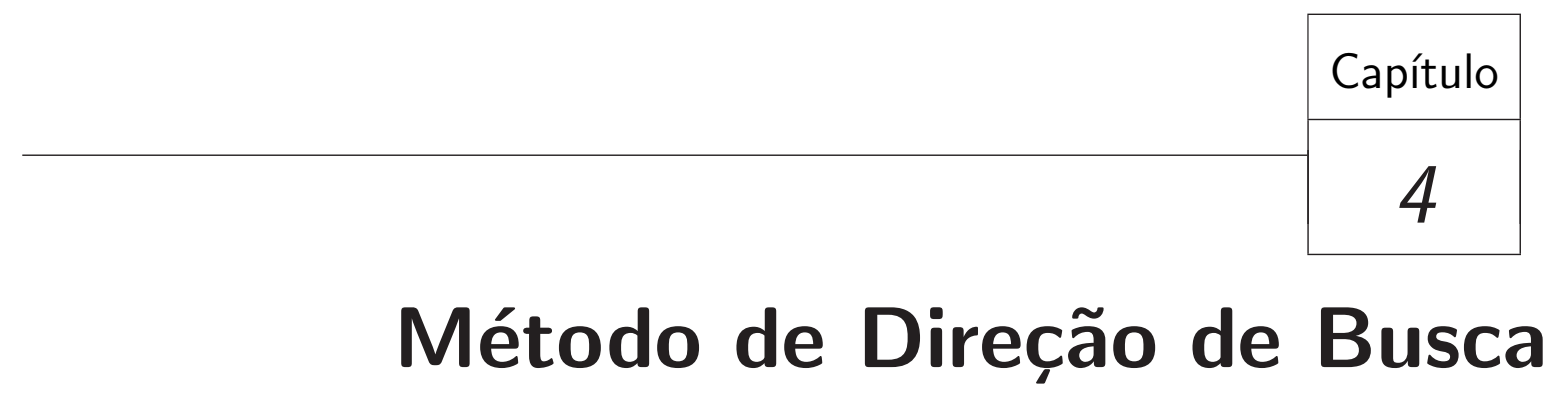

O problema de otimização formulado na Equação 2.26 consiste em um problema de otimização não-linear, submetido a uma restrição. Os problemas de otimização não-lineares são caracterizados por possuírem ao menos uma função não-linear na formulação matemática do problema (BAZARAA et al., 1979).

A resolução dos problemas de otimização não-lineares consiste em determinar uma solução factível, ou seja, uma solução que satisfaça todas as restrições do problema de forma a minimizar(maximizar) a função objetivo. Para resolver esse tipo de problema, deseja-se que os algoritmos gerem uma sequência de pontos que convirjam para a solução ótima. Porém, na maioria dos casos isto não ocorre devido a vários fatores, tais como: não convexidade da função objetivo, quantidade de variáveis, entre outros. Assim, em geral, os algoritmos desenvolvidos são capazes apenas de determinar uma solução ótima local em uma vizinhança, como o método de direção de busca.

O método de direção de busca para resolver o problema de minimizar(maximizar) uma função $f(x)$, onde $f: \mathbb{R}^{n} \rightarrow \mathbb{R}$ é diferenciável e contínua, consiste em: partir de um ponto inicial $x_{k}$ e determinar uma direção de busca $d_{k}$. Posteriormente, determina-se o tamanho do passo, $\alpha>0$, a ser dado ao longo da direção e obtém-se um novo ponto, $x_{k+1}=x_{k}+\alpha d_{k}$. Esse procedimento é repetido até que o critério de parada seja satisfeito. Para determinar o valor de $\alpha$ tem-se que resolver o sub problema de minimizar(maximizar) a função $h(\alpha)=f\left(x_{k}+\alpha d_{k}\right)$, o qual é um problema unidimensional na variável $\alpha$.

Há diversos algoritmos elaborados a partir do método de direção de busca que utilizam diferentes técnicas para determinar a direção de busca e o tamanho do passo. 
Neste trabalho, é apresentado o método de Newton, onde a direção de busca é determinada pela primeira e segunda derivadas da função $f$ e o tamanho do passo é igual a 1 em todas as iterações do algoritmo. O método do gradiente, cuja direção de busca é determinada pela primeira derivada da função $f$, também foi estudado para o problema do custo de T estágios. Porém, tal método apresentou resultados insatisfatórios, visto que, para os testes realizados o algoritmo divergiu ou atingiu o número máximo de iterações sem que a norma do gradiente em relação ao ganho obtido atendesse a tolerância adotada. Por este motivo, o método do gradiente será omitido.

Neste capítulo é apresentada na Seção 4.1 uma breve descrição, baseada em Bazaraa et al. (1979), do Método de Newton que resulta em uma sequência de ganhos $\mathbf{g} \in \mathcal{G}$ para o custo de $\mathrm{T}$ estágios, $\mathcal{J}_{T}$. O Cálculo das Derivadas do Custo são determinadas na Seção 4.2. Exemplos Numéricos ilustram a utilização deste método na Seção 4.3.

\subsection{Método de Newton}

Considere o problema formulado na Equação 2.26

$$
\begin{array}{ll}
\min _{\mathbf{g} \in \mathcal{G}} & \mathcal{J}_{T}=\sum_{k=0}^{T-1} J_{k} \\
\text { s.a. }\left\{\begin{array}{l}
X_{i}(k+1)=\mathcal{T}_{\bar{A}, i}(X(k))+\Sigma_{i}(k), \quad k \geq 0, \\
X_{i}(0)=\pi_{i}(0) x_{0} x_{0}^{\prime}, \quad i \in \mathcal{N} .
\end{array}\right.
\end{array}
$$

onde a cadeia de Markov, $\theta$, não é observada, os operadores $\mathcal{T}$ e $\Sigma$ são dados pela Definição 2.3 e com $J_{k}=\langle X(k), \bar{C}\rangle$ sendo que $\bar{C}_{i}=C_{i}+g(k)^{\prime} D_{i} g(k) \in \mathbb{S}^{r+}$ para todo $k \in \mathcal{K}$ e $i \in \mathcal{N}$.

Note que o problema apresentado na Equação 4.1 pode ser reescrito na forma reduzida, eliminando a restrição. Formalmente tem-se

$$
\min _{\mathbf{g} \in \mathcal{S}} \mathcal{J}_{T}=\sum_{k=0}^{T-1}\left\langle\mathcal{T}_{\bar{A}}(X(k-1))+\Sigma(k-1), \bar{C}\right\rangle .
$$

O método de Newton para o problema da Equação 4.2 consiste em aproximar $\mathcal{J}_{T}(\mathbf{g})$ por uma função quadrática, $Q(\mathbf{g})$, em torno de um ponto dado, $\mathbf{g}^{(\eta)}$, e com o mínimo desta função aproximada é determinada uma nova aproximação. Este processo é repetido até que um critério de parada seja satisfeito.

Considere a expansão da função $\mathcal{J}_{T}(\mathrm{~g})$ por série de Taylor (GUIDORIZZI, 2011), até a segunda ordem, nas vizinhanças do ponto $\mathbf{g}^{(\eta)}$ :

$$
\mathcal{J}_{T}(\mathbf{g}) \cong Q(\mathbf{g})=\mathcal{J}_{T}\left(\mathbf{g}^{(\eta)}\right)+\nabla \mathcal{J}_{T}\left(\mathbf{g}^{(\eta)}\right)^{\prime}\left(\mathbf{g}-\mathbf{g}^{(\eta)}\right)+\frac{1}{2}\left(\mathbf{g}-\mathbf{g}^{(\eta)}\right)^{\prime} H\left(\mathbf{g}^{(\eta)}\right)\left(\mathbf{g}-\mathbf{g}^{(\eta)}\right),
$$


onde $\nabla \mathcal{J}_{T}\left(\mathbf{g}^{(\eta)}\right)$ e $H\left(\mathbf{g}^{(\eta)}\right)$ são o vetor gradiente e a matriz hessiana da função $\mathcal{J}_{T}$ avaliadas no ponto $\mathbf{g}^{(\eta)}$.

Uma condição necessária para encontrar o mínimo da função $Q(\mathrm{~g})$ é que a derivada de $Q(\mathbf{g})$ com relação a variável $\mathbf{g}$ seja igual a zero. Assim, tem-se que

$$
\nabla \mathcal{J}_{T}\left(\mathbf{g}^{(\eta)}\right)+H\left(\mathbf{g}^{(\eta)}\right)\left(\mathbf{g}-\mathbf{g}^{(\eta)}\right)=0 .
$$

Logo, é possível determinar a sequência de pontos do método de Newton da seguinte forma:

$$
\begin{aligned}
& \text { i) Resolva o sistema linear: } H\left(\mathbf{g}^{(\eta)}\right) \mathbf{d}_{\eta}=-\nabla \mathcal{J}_{T}\left(\mathbf{g}^{(\eta)}\right) \text {, } \\
& \text { ii) Calcule } \mathbf{g}^{(\eta+1)}=\mathbf{g}^{(\eta)}-\mathbf{d}_{\eta} \text {. }
\end{aligned}
$$

Assumindo que $\nabla \mathcal{J}_{T}\left(\mathbf{g}^{*}\right)=0$ e que $H\left(\mathbf{g}^{*}\right)$ é definida positiva no mínimo local $\mathbf{g}^{*}$, e que $\partial_{T}$ possui derivadas parciais de primeira e segunda ordem contínuas, segue que $H\left(\mathbf{g}^{(\eta)}\right)$ é definida positiva nos pontos próximos a $\mathbf{g}^{*}$, assim o ponto sucessor $\mathbf{g}^{(\eta+1)}$ é bem definido.

\subsubsection{Modificação nos autovalores de $H$}

Em geral, o método de Newton pode não ser bem definido, pois a matriz $H$ pode não ser definida positiva ou estar perto de ser singular em um ponto $\mathbf{g}^{(\eta)}$. Neste caso, Bazaraa et al. (1979) e Luenberger (1984) recomendam utilizar uma aproximação para a matriz $H$.

Considere a matriz $B_{\eta}=H\left(\mathbf{g}^{(\eta)}\right)+\mu_{\eta} I$ onde $\mu_{\eta} \geq 0$ é o menor escalar tal que todos os autovalores da matriz $B_{\eta}$ sejam positivos. Assim, fixado $\delta>0$ tem-se que $\mu_{\eta}=$ $\delta-\lambda_{\text {min }}$, onde $\lambda_{\text {min }}$ é o menor autovalor de $H\left(\mathbf{g}^{(\eta)}\right)$. Desta forma, todos os autovalores de $B_{\eta}$ serão maiores ou iguais a $\delta$, ou seja, serão positivos e, consequentemente, $B_{\eta}$ será definida positiva e inversível.

No Algoritmo 4.1 é apresentado o método de Newton modificado que resulta a sequência de ganhos $\mathrm{g}^{*}$ que minimiza localmente o custo $\mathcal{J}_{T}$. Nos testes realizados, foi possível observar que se o ganho inicial $\mathbf{g}^{(0)}$ for escolhido aleatoriamente, então a sequência $\mathbf{g}^{(\eta)}$ gerada pelo método diverge ou as grandezas (segundo momento, derivadas e/ou custo) resultam em valores tão altos que não podem ser representadas no computador utilizado. Em virtude desta dificuldade, neste trabalho adotou-se a seguinte estratégia para escolha do ganho inicial.

Obtém-se a sequência de ganhos $K^{1}, \ldots, K^{N}$ pela equação recursiva de Riccati, ERR, (Apêndice B) para o problema onde $\theta(k)$ é observado. Considera-se o ganho 
$K^{N+1}$ cujos elementos são iguais a $K_{i j}^{N+1}=\left(\sum_{\ell=1}^{N} K_{i j}^{\ell}\right) / N$, para todo $i=1, \ldots, s$ e $j=1, \ldots, r$. Para cada $\ell=1, \ldots, N+1$ calcula-se o gradiente $\nabla \mathcal{J}_{T}\left(\mathbf{g}_{\ell}\right)$, sendo que $g_{\ell}(k)=K^{\ell}$ para todo $k \in \mathcal{K}$. A sequência de ganhos $\mathbf{g}^{(0)}$ será igual a sequência $\mathbf{g}_{\mathbf{i}}$ tal que $\left\|\nabla \mathcal{J}_{T}\left(\mathbf{g}_{\mathbf{i}}\right)\right\| \leq\left\|\nabla \mathcal{J}_{T}\left(\mathbf{g}_{\ell}\right)\right\|$.

Além disso, adota-se $\delta=-0,1 \lambda_{\min }\left[H\left(\mathbf{g}^{(\eta)}\right)\right]+1$ e utiliza-se, para fins de comparação com o método variacional, o método do gradiente biconjugado (disponibilizado no toolbox do $\left.M A T L A B^{\circledR}\right)$ para resolver o sistema linear apresentado no passo 2.

Algoritmo 4.1 Método de Newton Modificado

Passo 0: Inicie o processo em $\eta=0$ e escolha uma sequência de ganhos inicial $\mathbf{g}^{(\eta)}$.

Passo 1: Se $\lambda_{\min }\left[H\left(\mathbf{g}^{(\eta)}\right)\right]>0$ então $B_{\eta}=H\left(\mathbf{g}^{(\eta)}\right)$.

Caso contrário, $B_{\eta}=H\left(\mathbf{g}^{(\eta)}\right)+\mu_{\eta} I$ sendo que $\mu_{\eta}=\delta-\lambda_{\min }\left[H\left(\mathbf{g}^{(\eta)}\right)\right]$.

Passo 2: Resolva o sistema linear: $B_{\eta} \mathbf{d}_{\eta}=-\nabla \mathcal{J}_{T}\left(\mathbf{g}^{(\eta)}\right)$.

Passo 3: Faça $\mathbf{g}^{(\eta+1)}=\mathbf{g}^{(\eta)}+\mathbf{d}_{\eta}$.

Critério de Parada: $\left\|\nabla \mathcal{J}_{T}\left(\mathbf{g}^{(\eta)}\right)\right\|<\epsilon$ ou $\left|\mathcal{J}_{T}\left(\mathbf{g}^{(\eta)}\right)-\mathcal{J}_{T}\left(\mathbf{g}^{(\eta-1)}\right)\right| / \mathcal{J}_{T}\left(\mathbf{g}^{(\eta)}\right)<\epsilon$, para $\epsilon$ dado. Se o critério não for satisfeito, volte para o passo 1.

\subsection{Cálculo das Derivadas do Custo}

Nesta seção são apresentados o cálculo analítico das derivadas do custo de $\mathrm{T}$ estágios, desenvolvido neste trabalho, assim como o cálculo numérico das mesmas, baseado fortemente em Kincaid e Cheney (1991).

\subsubsection{Cálculo Analítico das Derivadas}

Os resultados a seguir apresentam a determinação da primeira e segunda derivadas do custo por estágio $J_{k}(g(k)), \forall k \in \mathcal{K}$. Visto que, pelas propriedades do cálculo diferencial, tem-se

$$
\frac{\partial \mathcal{J}_{T}}{\partial g}=\left[\frac{\partial J_{0}}{\partial g(0)} \cdots \frac{\partial J_{T-1}}{\partial g(T-1)}\right]^{\prime} \quad \text { e } \frac{\partial^{2} \mathcal{J}_{T}}{\partial g g^{\prime}}=\left[\begin{array}{ccc}
\frac{\partial^{2} J_{0}}{\partial g(0) g(0)^{\prime}} & \cdots & 0 \\
\vdots & \ddots & \vdots \\
0 & \cdots & \frac{\partial^{2} J_{T-1}}{\partial g(T-1) g(T-1)^{\prime}}
\end{array}\right]
$$

sendo que $g=[g(0) \ldots g(T-1)]^{\prime} \operatorname{com} g(k) \in \mathbf{g}$ para todo $k \in \mathcal{K}$. 
Lema 4.1. Considere $X(k)$ definido na Equação 2.23 e o ganho $g(k) \in \mathbf{g}$. Então

(i) a Matriz Jacobiana de $X(k)$ em relação a $g(k)$, de dimensão $r r \times r s$, é dada por

$$
\dot{X}(k)=\frac{\partial X(k)}{\partial g(k)}=\frac{\partial\left[\mathcal{T}_{\bar{A}}(X(k-1))\right]}{\partial g(k)},
$$

(ii) e a Matriz Hessiana de $X(k)$ em relação a $g(k)$, de dimensão rrrs $\times$ rs, é dada por

$$
\ddot{X}(k)=\frac{\partial^{2} X(k)}{\partial g(k) g(k)^{\prime}}=\frac{\partial^{2}\left[\mathcal{T}_{\bar{A}}(X(k-1))\right]}{\partial g(k) g(k)^{\prime}} .
$$

Demonstração. (i) Pela Equação 2.23 tem-se que $X(k)=\mathcal{T}_{\bar{A}}(X(k-1))+\Sigma(k-1)$, para todo $k \in \mathcal{K}$. Então, a matriz jacobiana de $X(k)$ em relação a $g(k)$ é dada por

$$
\frac{\partial X(k)}{\partial g(k)}=\frac{\partial\left[\mathcal{T}_{\bar{A}}(X(k-1))\right]}{\partial g(k)}+\frac{\partial[\Sigma(k-1)]}{\partial g(k)}, \forall k \in \mathcal{K}
$$

Como a sequência de matrizes $\Sigma(k) \in \mathbb{S}^{r 0}$ não dependem da sequência de ganhos $\mathbf{g} \in \mathcal{G}$, obtém-se a Equação 4.7.

(ii) A prova é análoga a do item anterior.

Teorema 4.1. Considere o operador $\mathcal{T}_{\bar{A}, i}(V)$ dado pela Definição 2.3. Então,

(i) a Matriz Jacobiana de $\mathcal{T}_{\bar{A}, i}(V)$ em relação a $g(k)$, de dimensão $r r \times r s$, é dada por

$$
\frac{\partial \mathcal{T}_{\bar{A}, i}(V)}{\partial g(k)}=\sum_{j=1}^{N} p_{j i}\left[\left(\bar{A}_{j} V_{j} \otimes B_{j}\right)+\left(\bar{A}_{j} \otimes \bar{A}_{j}\right) \frac{\partial V_{j}}{\partial g(k)}+\left(B_{j} \otimes \bar{A}_{j} V_{j}\right) \mathcal{P}_{s, r}\right]
$$

(ii) e a Matriz Hessiana de $\mathcal{T}_{\bar{A}, i}(V)$ em relação a $g(k)$, de dimensão $r r r s \times r s$, é dada por

$$
\begin{aligned}
& \frac{\partial^{2} \mathcal{T}_{\bar{A}, i}(V)}{\partial g(k) g(k)^{\prime}}=\sum_{j=1}^{N} p_{j i}\left[(\alpha+\beta)\left(\left(\bar{A}_{j} \otimes I^{r}\right) \frac{\partial V_{j}}{\partial g(k)}+\left(B_{j} \otimes V_{j}\right) \mathcal{P}_{s, r}\right)\right. \\
& \left.+\left(\bar{A}_{j} \otimes \bar{A}_{j} \otimes I^{r s}\right) \frac{\partial^{2} V_{j}}{\partial g(k) g(k)^{\prime}}+\left(I^{r r} \otimes\left(\frac{\partial V_{j}}{\partial g(k)}\right)^{\prime}\right) \gamma\left(B_{j} \otimes I^{r}\right) \mathcal{P}_{s, r}\right]
\end{aligned}
$$

onde $\alpha=\left(I^{r} \otimes\left[\left(\mathcal{P}_{r, r} \otimes I^{s}\right)\left(I^{r} \otimes \operatorname{vec}\left(B_{j}^{\prime}\right)\right)\right]\right), \beta=\left(I^{r r} \otimes \mathcal{P}_{r, s}\right)\left(\left[\left(I^{r} \otimes \mathcal{P}_{r, s}\right)\left(\operatorname{vec}\left(B_{j}^{\prime}\right) \otimes\right.\right.\right.$ $\left.\left.\left.I^{r}\right)\right] \otimes I^{r}\right)$ e $\gamma=\left(I^{r} \otimes\left[\left(\mathcal{P}_{r, r} \otimes I^{r}\right)\left(I^{r} \otimes \operatorname{vec}\left(\bar{A}_{j}^{\prime}\right)\right)\right]\right)+\left(\left[\left(I^{r} \otimes \mathcal{P}_{r, r}\right)\left(\operatorname{vec}\left(\bar{A}_{j}^{\prime}\right) \otimes I^{r}\right)\right] \otimes I^{r}\right)$.

Demonstração. A prova está disponível no Apêndice A. 
Teorema 4.2. Considere o custo por estágio $J_{k}(g(k))=\left\langle X(k), C+g(k)^{\prime} D g(k)\right\rangle$ (definido na Equação 2.24) e $\lambda_{i}=\operatorname{vec}\left(C_{i}\right)^{\prime}+\operatorname{vec}\left(D_{i}\right)^{\prime}(g(k) \otimes g(k)), i \in \mathcal{N}$. Então,

(i) o Vetor Gradiente de $J_{k}(g(k))$ em relação a $g(k)$, de dimensão $1 \times$ rs, é dado por

$$
\frac{\partial J_{k}(g(k))}{\partial g(k)}=\sum_{i=1}^{N}\left[\lambda_{i} \dot{X}_{i}(k)+2 \operatorname{vec}(g(k))^{\prime}\left(X_{i}(k) \otimes D_{i}\right)\right]
$$

(ii) e a Matriz Hessiana de $J_{k}(g(k))$ em relação a $g(k)$, de dimensão $r s \times r s$, é dada por

$$
\frac{\partial^{2} J_{k}(g(k))}{\partial g(k) g(k)^{\prime}}=\sum_{i=1}^{N}\left[\left(\lambda_{i} \otimes I^{r s}\right) \ddot{X}_{i}(k)+\left(\dot{X}_{i}(k)\right)^{\prime}\left(\operatorname{vec}\left(D_{i}\right)^{\prime} \otimes I^{r r}\right) \mu_{i}+2 \tau_{i}\right],
$$

onde $\mu_{i}=I^{s} \otimes\left[\left(\mathcal{P}_{s, r} \otimes I^{r}\right)\left(I^{r} \otimes \operatorname{vec}\left(g(k)^{\prime}\right)\right)\right]+\left[\left(I^{s} \otimes \mathcal{P}_{s, r}\right)\left(\operatorname{vec}\left(g(k)^{\prime}\right) \otimes I^{s}\right)\right] \otimes I^{r}$ $e \tau_{i}=\left(\operatorname{vec}(g(k))^{\prime} \otimes I^{r s}\right)\left(I^{r} \otimes\left[\left(\mathcal{P}_{s, r} \otimes I^{s}\right)\left(I^{r} \otimes \operatorname{vec}\left(D_{i}\right)\right)\right]\right) \dot{X}_{i}(k)+\left(X_{i}(k) \otimes D_{i}\right)$.

Demonstração. A prova está disponível no Apêndice A.

Observação 4.1. O cálculo analítico das derivadas do custo de T estágios não é viável computacionalmente, principalmente para horizontes grandes e/ou problemas de grande porte. Assim, nas implementações realizadas utilizou-se o cálculo numérico das derivadas do custo pelo método das Diferenças Aproximadas Centrais (KINCAID, CHENEY, 1991) descrito a seguir.

\subsubsection{Cálculo Numérico das Derivadas}

Considere uma função $f: \mathbb{R} \rightarrow \mathbb{R}$. Pela expansão de $f$ por série de Taylor, tem-se que

$$
\begin{aligned}
& f(x+h)=f(x)+h f^{(1)}(x)+\frac{h^{2}}{2} f^{(2)}(x)+\frac{h^{3}}{6} f^{(3)}\left(\xi_{1}\right)+\frac{h^{4}}{24} f^{(4)}\left(\bar{\xi}_{1}\right), \\
& f(x-h)=f(x)-h f^{(1)}(x)+\frac{h^{2}}{2} f^{(2)}(x)-\frac{h^{3}}{6} f^{(3)}\left(\xi_{2}\right)+\frac{h^{4}}{24} f^{(4)}\left(\bar{\xi}_{2}\right),
\end{aligned}
$$

sendo que $h$ é um número suficientemente pequeno, $\xi_{1}, \bar{\xi}_{1} \in(x, x+h)$ e $\xi_{2}, \bar{\xi}_{2} \in(x-h, x)$.

Subtraindo a Equação 4.13 de 4.12 , obtém-se a seguinte aproximação para a primeira derivada da função $f$

$$
f^{(1)}(x)=\frac{f(x+h)-f(x-h)}{2 h}-\frac{h^{2}}{12}\left[f^{(3)}\left(\xi_{1}\right)+f^{(3)}\left(\xi_{2}\right)\right] .
$$

Note que a Equação 4.14 será válida somente se as funções $f$ e $f^{(1)}$ forem contínuas no intervalo $(x-h, x+h)$, sendo que a terceira derivada de $f$ deve existir neste intervalo. 
Além disso, observe que o erro na Equação 4.14 é proporcional ao valor de $h$ e de $f^{(3)}$. O termo $h$ na equação faz com que o erro convirja para zero conforme $h$ tende a zero. Assim, a Equação 4.14 apresenta uma boa aproximação da derivada de $f$, visto que a sua convergência, dada pela potência de $h$, é quadrática.

Somando as Equações 4.13 e 4.12, obtém-se a aproximação para a segunda derivada de $f$

$$
f^{(2)}(x)=\frac{f(x+h)-2 f(x)+f(x-h)}{2 h}-\frac{h^{2}}{12}\left[f^{(4)}\left(\bar{\xi}_{1}\right)+f^{(4)}\left(\bar{\xi}_{2}\right)\right],
$$

sendo que $f^{(4)}$ deve existir no intervalo $(x-h, x+h)$. Observe que a convergência desta aproximação também é quadrática.

Para o caso de uma função $f: \mathbb{R}^{n} \rightarrow \mathbb{R}$ a aproximação é feita de modo análogo. Considere $n=2$, então a aproximação da derivada parcial com relação à $x_{1}$, por exemplo, pode ser obtida fixando o valor de $x_{2}$, assim tem-se uma função unidimensional. Logo,

$$
\frac{\partial f\left(x_{1}, x_{2}\right)}{\partial x_{1}} \approx \frac{f\left(x_{1}+h, x_{2}\right)-f\left(x_{1}-h, x_{2}\right)}{2 h} .
$$

Além disso, as segundas derivadas de $f\left(x_{1}, x_{2}\right)$ são dadas por

$$
\begin{aligned}
\frac{\partial^{2} f\left(x_{1}, x_{2}\right)}{\partial x_{1}^{2}} \approx & \frac{f\left(x_{1}+h, x_{2}\right)-2 f\left(x_{1}, x_{2}\right)+f\left(x_{1}-h, x_{2}\right)}{h^{2}} . \\
\frac{\partial^{2} f\left(x_{1}, x_{2}\right)}{\partial x_{2}^{2}} \approx & \frac{f\left(x_{1}, x_{2}+w\right)-2 f\left(x_{1}, x_{2}\right)+f\left(x_{1}, x_{2}-w\right)}{w^{2}} . \\
\frac{\partial^{2} f\left(x_{1}, x_{2}\right)}{\partial x_{1} x_{2}} \approx & \frac{f\left(x_{1}+h, x_{2}+w\right)-f\left(x_{1}-h, x_{2}+w\right)}{4 h w}- \\
& \frac{f\left(x_{1}+h, x_{2}-w\right)+f\left(x_{1}-h, x_{2}-w\right)}{4 h w} .
\end{aligned}
$$

Portanto, para determinar a derivada numérica do custo de $\mathrm{T}$ estágios basta calcular a derivada aproximada do custo por estágio $J_{k}$, para cada valor de $k \in \mathcal{K}$ (veja Equação 4.6), utilizando a aproximação descrita para a função $f: \mathbb{R}^{n} \rightarrow \mathbb{R}\left(f:=J_{k} \mathrm{e}\right.$ $x:=g(k))$.

Observação 4.2. O método de direção de busca pode ser utilizado para obter um ganho $g \in \mathcal{M}^{s, r}$ que minimiza localmente o problema do CMLP (Equação 2.31). Basta considerar uma aproximação do CMLP via o CHF, onde T é suficientemente grande (COSTA et al., 2011). Neste caso, considera-se o problema de determinar o ganho $g \in \mathcal{M}^{s, r}$ que minimiza $J_{T}(g)$. 


\subsection{Exemplos Numéricos}

Nesta seção são apresentados exemplos numéricos para ilustrar a utilização do método de direção de busca cujo resultado pode ser uma aproximação para a solução do problema do custo de T estágios (Equação 4.1).

Exemplo 4.1. Considere os seguintes parâmetros para o SLSM $\Phi$ (Equação 2.11),

$$
\begin{aligned}
& A_{1}=\left[\begin{array}{rrr}
-1,1785 & 0,1366 & 0,8792 \\
1,3384 & -0,1248 & -1,5338 \\
-0,8852 & 0,1278 & 0,5903
\end{array}\right], A_{2}=\left[\begin{array}{rrr}
-0,3094 & 1,0065 & 0,2539 \\
-0,5622 & 1,2145 & 0,0466 \\
0,1835 & -0,2072 & 0,3477
\end{array}\right] \\
& C_{1}=\left[\begin{array}{rrc}
3,6832 & 0,3376 & -4,0719 \\
0,3376 & 0,0309 & -0,3732 \\
-4,0719 & -0,3732 & 4,5016
\end{array}\right], C_{2}=\left[\begin{array}{rrr}
0,4598 & -0,7323 & -0,2889 \\
-0,7323 & 1,1662 & 0,4600 \\
-0,2889 & 0,4600 & 0,1815
\end{array}\right] \text {, } \\
& B_{1}=\left[\begin{array}{r}
-0,5255 \\
1,5146 \\
0,4058
\end{array}\right], B_{2}=\left[\begin{array}{r}
1,5393 \\
1,7570 \\
-1,7212
\end{array}\right], G_{1}=\left[\begin{array}{r}
-2,2320 \\
1,1367 \\
-1,4398
\end{array}\right], G_{2}=\left[\begin{array}{r}
0,0926 \\
0,3402 \\
-0,6206
\end{array}\right] \text {, } \\
& \mathbb{P}=\left[\begin{array}{ll}
0,8133 & 0,1867 \\
0,1478 & 0,8522
\end{array}\right], \pi(0)=\left[\begin{array}{ll}
0,7261 & 0,2739
\end{array}\right], x_{0}=\left[\begin{array}{r}
2,4953 \\
0,8559 \\
-0,8510
\end{array}\right],
\end{aligned}
$$

$D_{1}=0,7837$ e $D_{2}=0,5341$.

O Algoritmo 4.1 foi implementado no software $M A T L A B^{\circledR}$ com tolerância $\epsilon=$ $10^{-4}$, horizonte $T=75$ e foi iniciado com a sequência de ganhos $\mathbf{g}^{(0)}$ tal que

$$
g^{(0)}(k)=K^{1} \approx\left[\begin{array}{lll}
-0,3029 & 0,0132 \quad 0,3241
\end{array}\right], \forall k \in \mathcal{K},
$$

visto que $\left\|\nabla \mathcal{J}_{T}\left(\mathbf{g}^{(0)}\right)\right\| \approx 50,4267$. O algoritmo realizou $\eta=6$ iterações, interrompendo o processo com o critério de parada igual a

$$
\left|\mathcal{J}_{T}\left(\mathbf{g}^{(\eta)}\right)-\mathcal{J}_{T}\left(\mathbf{g}^{(\eta-1)}\right)\right| / \mathcal{J}_{T}\left(\mathbf{g}^{(\eta)}\right) \approx 4,1117 \times 10^{-6} .
$$

O custo de T estágios obtido foi igual a

$$
\partial_{T}\left(\mathbf{g}^{*}\right) \approx 217,5032
$$

Na Figura 4.1 são apresentados os comportamentos da norma de 6 ganhos $\|g(k)\|, k \in$ $\mathcal{K}$, da norma do segundo momento $X(T)$, da norma do gradiente $\nabla \mathcal{J}_{T}$ e do custo de T estágios $\mathcal{J}_{T}$, em função do número de iterações $\eta$. Note que a sequência de pontos em todos os gráficos convergem conforme o número de iterações, $\eta$, aumenta. Além disso, como era tipicamente esperado, a cada iteração a norma do gradiente decresce, tendendo a zero. 


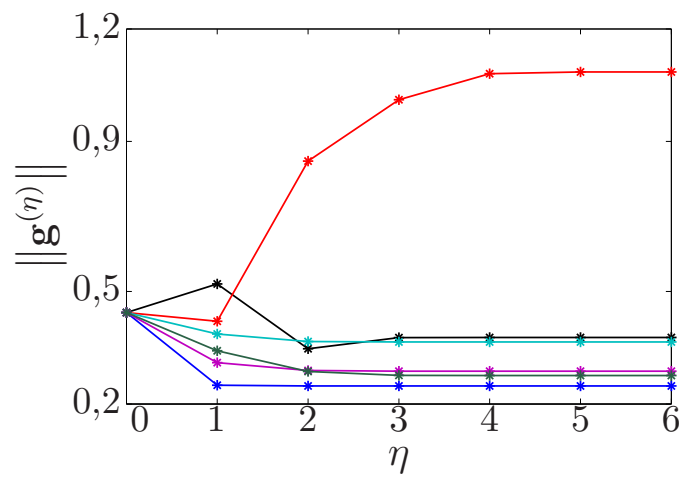

(a) Norma de g versus $\eta$

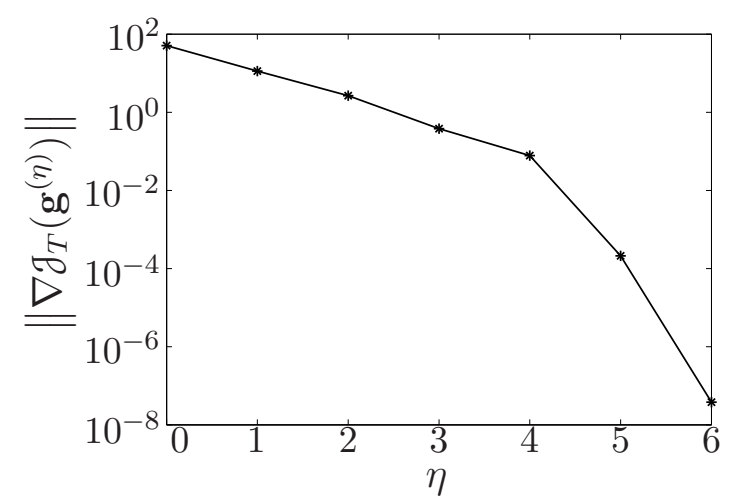

(c) Norma de $\nabla \mathcal{J}_{T}$ versus $\eta$

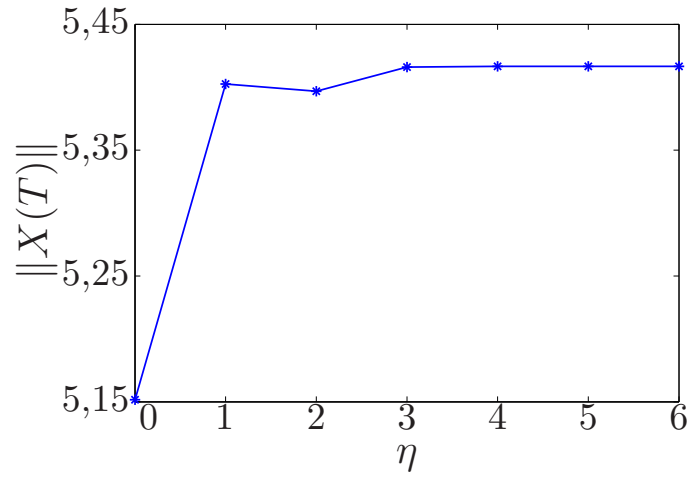

(b) Norma de $X(T)$ versus $\eta$

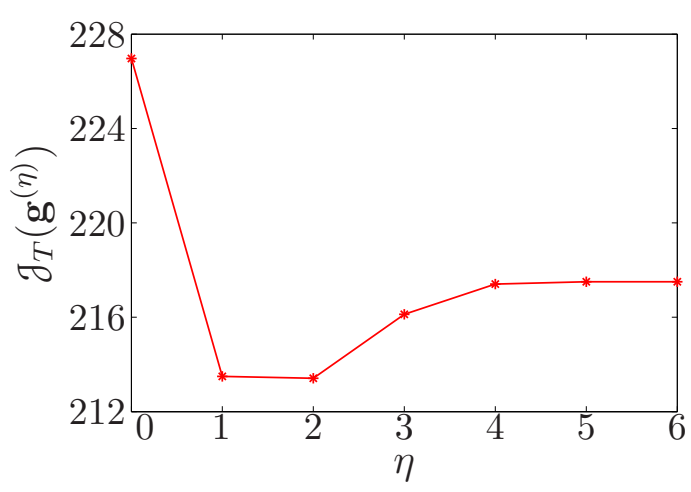

(d) Custo $\mathcal{J}_{T}$ versus $\eta$

Figura 4.1: Resultados do método de Newton modificado para o Exemplo 4.1

Observação 4.3. Para ilustrar a inviabilidade de se utilizar o cálculo analítico das derivadas na implementação do algoritmo (Observação 4.1), o Exemplo 4.1 também foi resolvido utilizando este cálculo. Neste caso, tem-se que $\mathcal{J}_{T}\left(\mathbf{g}^{*}\right)=217,5032$, porém o tempo computacional despendido foi igual a $t_{A}=156,8122$ segundos enquanto $o$ método numérico leva $t_{N}=27,9398$ segundos, sendo que o erro é da ordem de $10^{-6}$.

A estratégia adotada para escolher o ganho inicial, $\mathbf{g}^{(0)}$, nem sempre resulta um ganho adequado para inicializar o método de Newton. Neste caso, uma alternativa para o problema do custo de T estágios é utilizar outra sequência inicial de ganhos. Contudo, esta não é uma tarefa simples, visto que não se tem informações sobre o comportamento do custo $\mathcal{J}_{T}$. Uma opção encontrada, consiste em perturbar a sequência inicial de ganhos, $\mathbf{g}^{(0)}$, a fim de determinar uma nova, $\overline{\mathbf{g}}^{(0)}$, tal que $\left\|\nabla \mathcal{J}_{T}\left(\overline{\mathbf{g}}^{(0)}\right)\right\|<$ $\left\|\nabla \mathcal{J}_{T}\left(\mathbf{g}^{(0)}\right)\right\|$. No Exemplo 4.2 é apresentado um problema no qual o método diverge quando é iniciado com o ganho, $\mathbf{g}^{(0)}$, escolhido pela estratégia adotada. Porém, no Exemplo 4.3 o algoritmo converge quando é iniciado com o ganho, $\overline{\mathbf{g}}^{(0)}$, escolhido pela estratégia alternativa. 
Exemplo 4.2. Considere os seguintes parâmetros para o SLSM $\Phi$ (Equação 2.11),

$$
\begin{aligned}
& A_{1}=\left[\begin{array}{rrr}
0,5071 & -0,9974 & 0,3509 \\
0,0672 & 1,0010 & -0,0813 \\
0,1669 & -0,2388 & 0,4707
\end{array}\right], A_{2}=\left[\begin{array}{rrr}
-0,6362 & -0,9552 & -0,6964 \\
-0,0088 & -1,3210 & -0,5113 \\
0,2373 & 1,8700 & 0,8932
\end{array}\right] \text {, } \\
& C_{1}=\left[\begin{array}{rrr}
0,4276 & -1,2242 & 0,4776 \\
-1,2242 & 3,5048 & -1,3672 \\
0,4776 & -1,3672 & 0,5334
\end{array}\right], C_{2}=\left[\begin{array}{lll}
0,7449 & 2,2491 & 2,0344 \\
2,2491 & 6,7905 & 6,1421 \\
2,0344 & 6,1421 & 5,5556
\end{array}\right] \text {, } \\
& B_{1}=\left[\begin{array}{l}
0,5148 \\
0,0055 \\
0,4510
\end{array}\right], B_{2}=\left[\begin{array}{r}
-0,0763 \\
0,3584 \\
0,0103
\end{array}\right], G_{1}=\left[\begin{array}{r}
0,1703 \\
0,2037 \\
-0,2407
\end{array}\right], G_{2}=\left[\begin{array}{r}
1,5523 \\
1,0771 \\
-3,2965
\end{array}\right] \text {, } \\
& \mathbb{P}=\left[\begin{array}{ll}
0,7129 & 0,2871 \\
0,6998 & 0,3002
\end{array}\right], \pi(0)=\left[\begin{array}{ll}
0,3893 & 0,6107
\end{array}\right], x_{0}=\left[\begin{array}{r}
0,3394 \\
-0,1311 \\
0,4852
\end{array}\right],
\end{aligned}
$$

$D_{1}=0,9828$ e $D_{2}=0,4022$.

O Algoritmo 4.1 foi implementado com tolerância $\epsilon=10^{-4}$, horizonte $T=65 \mathrm{e}$ foi iniciado com a sequência de ganhos $\mathbf{g}^{(0)}$ tal que

$$
g^{(0)}(k)=K^{1} \approx\left[\begin{array}{lll}
-0,3667 & 0,1930 & -0,4982
\end{array}\right], \forall k \in \mathcal{K},
$$

visto que $\left\|\nabla \mathcal{J}_{T}\left(\mathbf{g}^{(0)}\right)\right\| \approx 233,4407$. O algoritmo realizou $\eta=3$ iterações, interrompendo o processo devido à norma do gradiente para a sequência de ganhos $\mathbf{g}^{(3)}$ ser da ordem de $10^{169}$. O custo de T estágios obtido foi igual a

$$
\mathcal{J}_{T}\left(\mathbf{g}^{(3)}\right) \approx 1,0384 \times 10^{16}
$$

Na Figura 4.2 são apresentados os comportamentos da norma de 6 ganhos $\|g(k)\|, k \in$ $\mathcal{K}$, da norma do segundo momento $X(T)$, da norma do gradiente $\nabla \mathcal{J}_{T}$ e do custo de $\mathrm{T}$ estágios $\mathcal{J}_{T}$, em função do número de iterações $\eta$. Note que a sequência de pontos em todos os gráficos divergem conforme o número de iterações, $\eta$, aumenta. 


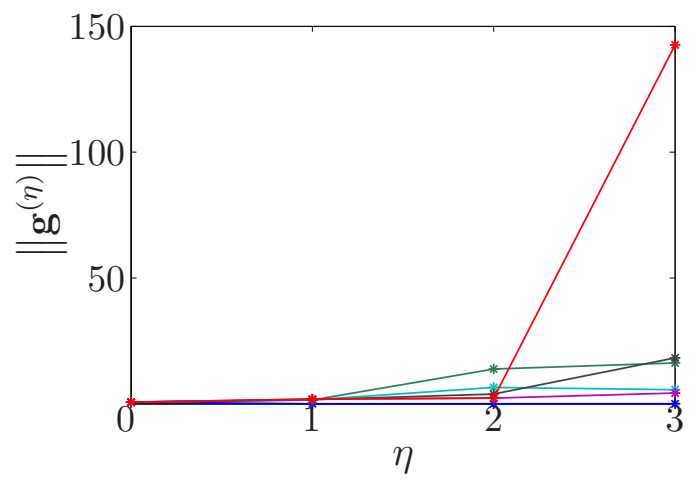

(a) Norma de g versus $\eta$

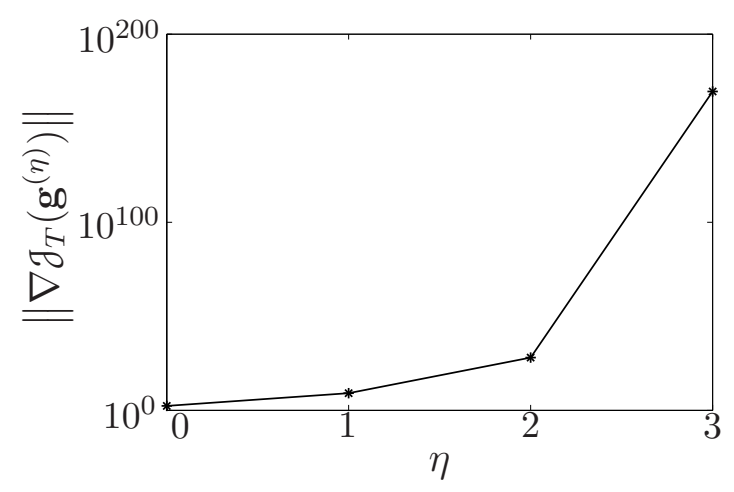

(c) Norma de $\nabla \mathcal{J}_{T}$ versus $\eta$

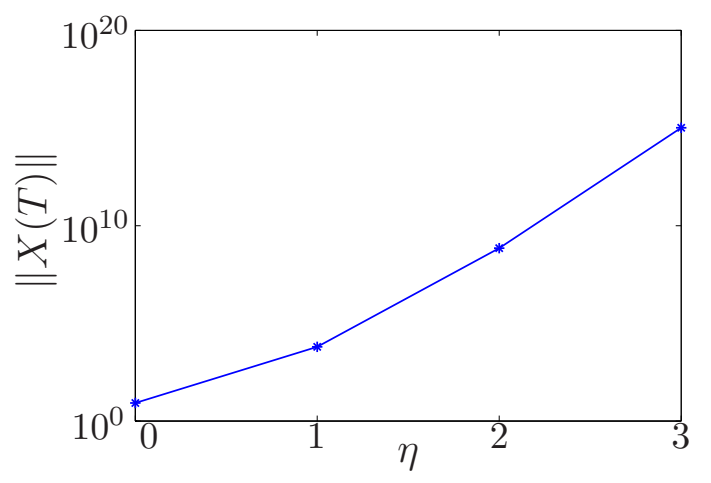

(b) Norma de $X(T)$ versus $\eta$

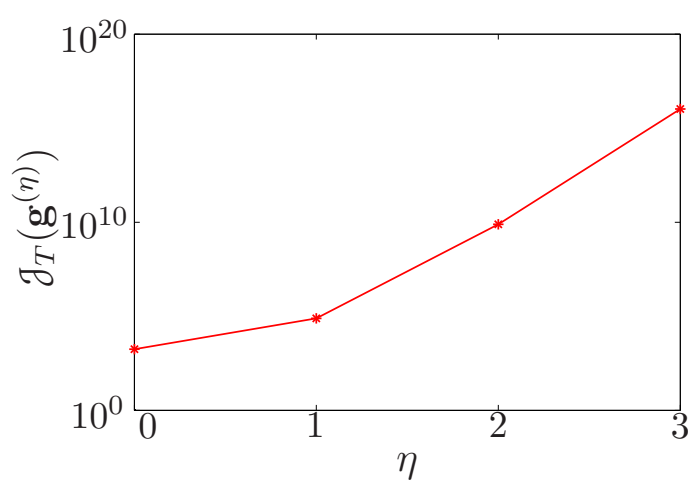

(d) Custo $\mathcal{J}_{T}$ versus $\eta$

Figura 4.2: Resultados do método de Newton modificado para o Exemplo 4.2

Exemplo 4.3. Considere os parâmetros dados no Exemplo 4.2 para o SLSM $\boldsymbol{\Phi}$ (Equação 2.11) e a sequência inicial de ganhos $\overline{\mathbf{g}}^{(0)}$ tal que

$$
\bar{g}^{(0)}(k) \approx\left[\begin{array}{lll}
-0.3667+\xi & 0.1930+\xi & -0.4982+\xi
\end{array}\right], \forall k \in \mathcal{K},
$$

sendo que $\xi=10^{-5}$ e $\left\|\nabla \mathcal{J}_{T}\left(\overline{\mathbf{g}}^{(0)}\right)\right\| \approx 233,4393$. O algoritmo realizou $\eta=168$ iterações, interrompendo o processo com o critério de parada igual a

$$
\left|\mathcal{\partial}_{T}\left(\overline{\mathbf{g}}^{(\eta)}\right)-\mathcal{J}_{T}\left(\overline{\mathbf{g}}^{(\eta-1)}\right)\right| / \mathcal{J}_{T}\left(\overline{\mathbf{g}}^{(\eta)}\right) \approx 7,3368 \times 10^{-5}
$$

O custo de T estágios obtido foi igual a

$$
\partial_{T}\left(\overline{\mathbf{g}}^{*}\right) \approx 1,4719 \times 10^{3} .
$$

Na Figura 4.3 são apresentados os comportamentos da norma de 4 ganhos $\|g(k)\|, k \in$ $\mathcal{K}$, da norma do segundo momento $X(T)$, da norma do gradiente $\nabla \mathcal{J}_{T}$ e do custo de T estágios $\mathcal{J}_{T}$, em função do número de iterações $\eta$. Note que apesar da sequência de pontos em todos os gráficos divergirem nas primeiras 20 iterações, nas posteriores convergem para uma solução. 


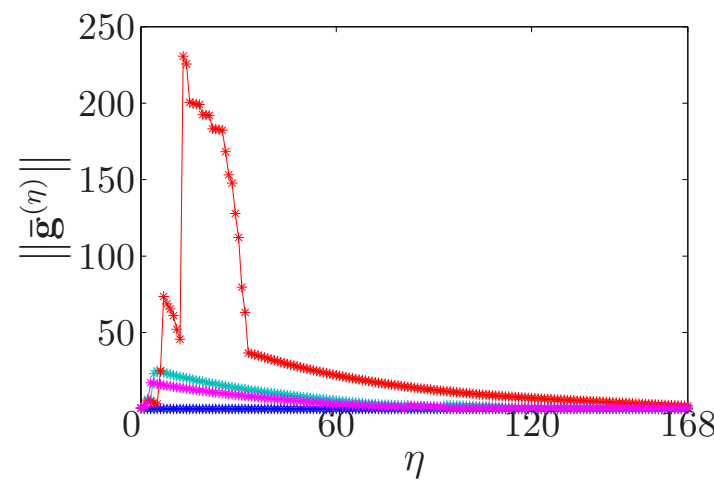

(a) Norma de $\overline{\mathbf{g}}$ versus $\eta$

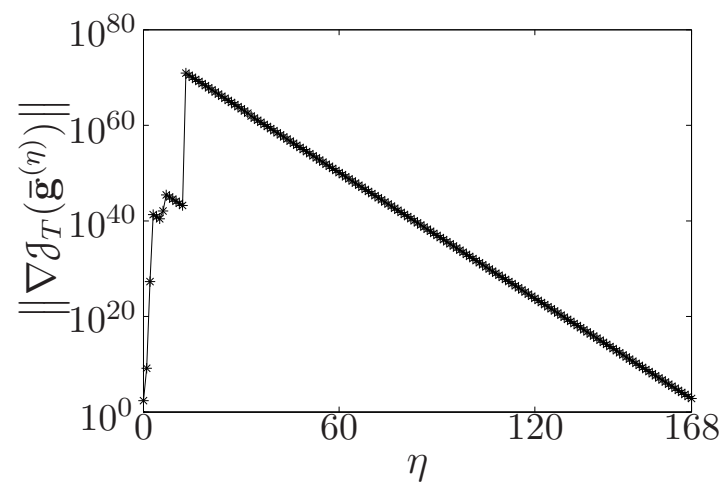

(c) Norma de $\nabla \mathcal{J}_{T}$ versus $\eta$

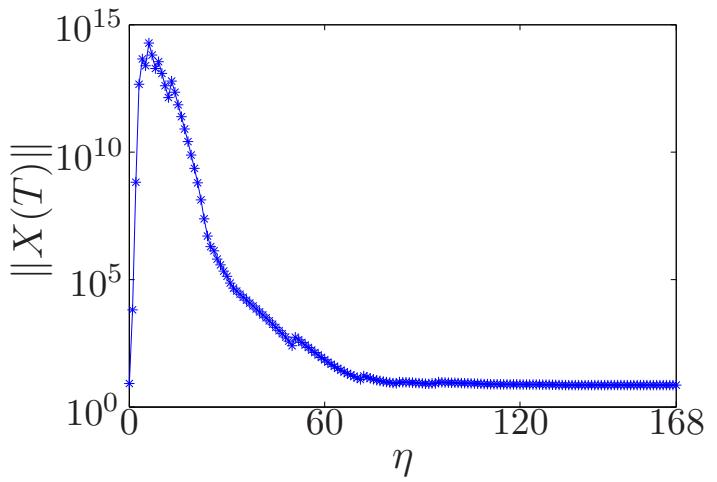

(b) Norma de $X(T)$ versus $\eta$

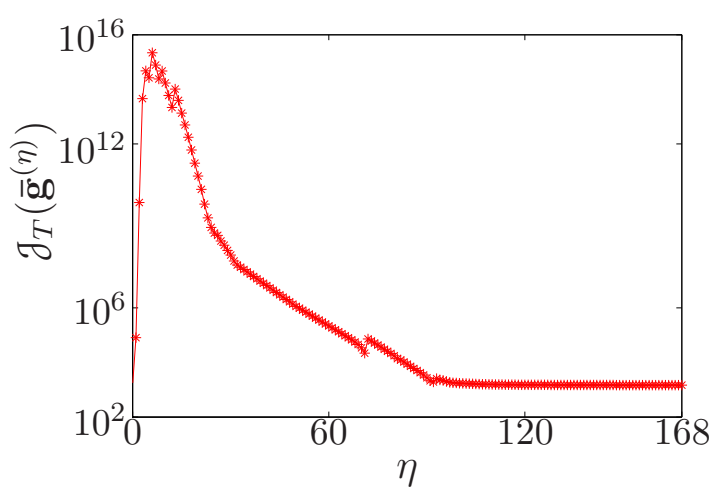

(d) Custo $\mathcal{J}_{T}$ versus $\eta$

Figura 4.3: Resultados do método de Newton modificado para o Exemplo 4.3 


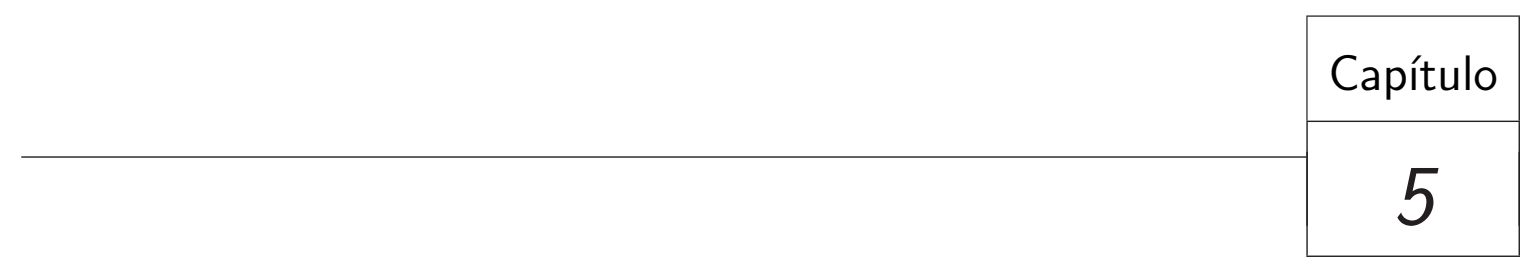

\section{Estratégia de Observação Indireta}

Nos capítulos anteriores, a variável de estado $\theta(k)$ não é observada em todo instante $k \in \mathcal{K}$, sendo que a única informação conhecida é a sua distribuição, $\pi(k)$. Neste capítulo propomos na Seção 5.1 uma metodologia que denominamos de Estratégia de Observação Indireta que apresenta um esquema de observação de $\theta(k)$, a partir de uma variável $r(k)$ que é perfeitamente observada para todo $k \in \mathcal{K}$. Este método introduz níveis intermediários de observação, partindo do cenário de observação completa (o que permite inicializá-lo com a solução da equação recursiva de Riccati) e lentamente alterando para o cenário de não observação. Na Seção 5.2 é descrito o Método Variacional Adaptado para a Observação Parcial. A Estratégia de Observação Indireta para o CMLP é apresentada na Seção 5.3. Exemplos Numéricos ilustram esta estratégia na Seção 5.4 .

Os resultados apresentados neste capítulo foram elaborados com a colaboração de Carlos Alexandre Silva, aluno do curso de doutorado em Ciências de Computação e Matemática Computacional do ICMC - USP.

\subsection{Estratégia de Observação Indireta}

Considere o SLSM definido na Equação 2.11

$$
\Phi: \begin{cases}x_{k+1}=A_{\theta(k)} x_{k}+B_{\theta(k)} u_{k}+G_{\theta(k)} w_{k}, & \forall k \geq 0, \\ y_{k}=x_{k}^{\prime} C_{\theta(k)} x_{k}+u_{k}^{\prime} D_{\theta(k)} u_{k}, & \theta(0) \sim \pi(0) .\end{cases}
$$

Suponha que a variável $r(k)$ dada a seguir é observada a cada instante de tempo $k \in \mathcal{K}$, trazendo informações sobre a variável $\theta(k)$, com distribuição condicional 


$$
\hat{p}_{i \ell}=P[r(k)=\ell \mid \theta(k)=i]=c \mathbb{1}_{\{i=\ell\}}+\frac{1-c}{N-1} \mathbb{1}_{\{i \neq \ell\}},
$$

sendo que $c \in\left[\frac{1}{N}, 1\right]$. Por exemplo, quando $c=1$ tem-se que $\theta(k)=r(k)$ com probabilidade 1 , o que corresponde ao cenário de observação completa. Para $c=\frac{1}{N}$, a distribuição de $\theta(k)$ não depende de $r(k)$, pois pela Regra de Bayes obtém-se que

$$
P[\theta=i \mid r=\ell]=\frac{P[r=\ell \mid \theta=i] P[\theta=i]}{\sum_{j \in \mathcal{N}} P[r=\ell \mid \theta=j] P[\theta=j]}=\frac{\frac{1}{N} \pi_{i}}{\sum_{j \in \mathcal{N}} \frac{1}{N} \pi_{j}}=\pi_{i}=P[\theta=i] .
$$

Logo, quando $c=\frac{1}{N}$, tem-se o cenário de não observação de $\theta(k)$. Os valores intermediários de $c \in\left(\frac{1}{N}, 1\right)$, são interpretados como diferentes Níveis de Observação para a variável $\theta$. De acordo com essa estrutura de observação, considera-se uma lei de controle linear na forma

$$
u_{k}=F_{r(k)}(k) x_{k} \quad \operatorname{com} \quad k \in \mathcal{K}
$$

sendo que $F_{r(k)}(k) \in \mathcal{M}^{s, r}$. Seja $\mathcal{F}$ o conjunto formado por todas as possíveis coleções de sequências de ganhos $\mathbf{F}=\left\{\mathbf{F}_{\mathbf{r}(\mathbf{0})}, \mathbf{F}_{\mathbf{r}(\mathbf{1})}, \ldots\right\} \operatorname{com} \mathbf{F}_{\mathbf{r}(\mathbf{k})}=\left\{F_{r(k)}(0), F_{r(k)}(1), \ldots\right\}$ para todo $k \in \mathcal{K}$. Substituindo a lei de controle da Equação 5.4 no SLSM $\Phi$ (Equação 5.1), obtém-se o seguinte SLSM:

$$
\hat{\boldsymbol{\Phi}}: \begin{cases}x_{k+1}=\hat{A}_{\theta(k), r(k)} x_{k}+G_{\theta(k)} w_{k}, & \forall k \geq 0, \\ y_{k}=x_{k}^{\prime} \hat{C}_{\theta(k), r(k)} x_{k}, & \theta(0) \sim \pi(0),\end{cases}
$$

sendo que $\hat{A}_{\theta(k), r(k)}=A_{\theta(k)}+B_{\theta(k)} F_{r(k)}(k) \in \mathcal{M}^{r}$ e $\hat{C}_{\theta(k), r(k)}=C_{\theta(k)}+F_{r(k)}(k)^{\prime} D_{\theta(k)} F_{r(k)}(k)$ $\in \mathcal{S}^{r+}$.

Observação 5.1. Note que o problema do custo de T estágios referente ao SLSM $\hat{\mathbf{\Phi}}$ (Equação 5.5) pode ser reformulado como na Equação 2.26 para o sistema $\overline{\mathbf{\Phi}}$ da Equação 2.21. Basta substituir, nas Equações 2.21 e 2.26, a variável $\theta(k)$ pelo novo estado $(\theta(k), r(k))$ com probabilidades de transição dadas por

$$
P[\theta(k+1)=j, r(k+1)=m \mid \theta(k)=i, r(k)=\ell]=p_{i j} \hat{p}_{j m} .
$$

Contudo a dimensão do problema aumenta (a dimensão de $\mathbb{P}$ torna-se $N^{2}$ ) e se torna computacionalmente bem mais pesado, o que motiva o desenvolvimento de uma formulação mais específica que preserva a dimensão de $\mathbb{P}$.

Os resultados a seguir apresentam uma formulação que permite utilizar a cadeia de Markov original para o problema do custo de T estágios com observação indireta da variável $\theta(k)$ e controle dado pela Equação 5.4. 
Definição 5.1. Considere as matrizes $\mathbb{P}=\left[p_{i j}\right] \in \mathcal{M}^{N}, G \in \mathbb{M}^{r, l}, V \in \mathbb{M}^{r}$ e $U \in \mathbb{S}^{r 0}$. Definem-se para todo $i \in \mathcal{N}$ e $k \in \mathcal{K}$ o operador linear $\hat{\mathcal{T}}_{V}: \mathbb{S}^{r 0} \rightarrow \mathbb{S}^{r 0}$ e a sequência de matrizes $\hat{\Sigma}(k) \in \mathbb{S}^{r 0}$ tais que:

$$
\begin{gathered}
\hat{\Sigma}_{i}(k)=\sum_{\ell=1}^{N} \sum_{j=1}^{N} p_{j i} \hat{p}_{j \ell} \pi_{j}(k) G_{j} G_{j}^{\prime}, \\
\hat{\mathcal{T}}_{V, i}(U)=\sum_{\ell=1}^{N} \sum_{j=1}^{N} p_{j i} \hat{p}_{j \ell} V_{j} U_{j} V_{j}^{\prime} .
\end{gathered}
$$

Para o operador $\hat{\mathcal{T}}_{V}$, define-se $\hat{\mathcal{T}}_{V}^{0}(U)=U$ e para $t \geq 1$ tem-se a recursã $\hat{\mathcal{T}}_{V}^{(t)}(U)=$ $\hat{\mathfrak{T}}_{V}\left(\hat{\mathfrak{T}}_{V}^{(t-1)}(U)\right)$.

Lema 5.1. Considere o SLSM $\hat{\boldsymbol{\Phi}}$ definido na Equação 5.5. Então, o segundo momento de $x_{k}$ condicionado ao estado markoviano $\theta(k)$ é dado por

$$
\left\{\begin{array}{l}
X(k+1)=\hat{\mathcal{T}}_{\hat{A}}(X(k))+\hat{\Sigma}(k), \quad k \geq 0, \\
X_{i}(0)=\pi_{i}(0) x_{0}^{\prime} x_{0}, \quad i \in \mathcal{N},
\end{array}\right.
$$

sendo que as matrizes $\hat{\mathcal{T}}_{\hat{A}}$ e $\hat{\Sigma}(k)$ dependem do nível de observação $c \in\left[\frac{1}{N}, 1\right]$.

Demonstração.

$$
\begin{aligned}
X_{i}(k+1)= & \sum_{\ell \in \mathcal{N}} E\left\{x_{k+1} x_{k+1}^{\prime} \mathbb{1}_{\{\theta(k+1)=i, r(k)=\ell\}}\right\} \\
= & \sum_{\ell \in \mathcal{N}} \sum_{j \in \mathcal{N}} E\left\{\left(\hat{A}_{\theta(k), r(k)} x_{k}+G_{\theta(k)} w_{k}\right)\left(\hat{A}_{\theta(k), r(k)} x_{k}+G_{\theta(k)} w_{k}\right)^{\prime} \times\right. \\
& \left.\mathbb{1}_{\{\theta(k+1)=i, r(k)=\ell, \theta(k)=j\}}\right\} \\
= & \sum_{\ell \in \mathcal{N}} \sum_{j \in \mathcal{N}} E\left\{\left(\hat{A}_{\theta(k), r(k)} x_{k}+G_{\theta(k)} w_{k}\right)\left(\hat{A}_{\theta(k), r(k)} x_{k}+G_{\theta(k)} w_{k}\right)^{\prime} \times\right. \\
& \left.\mathbb{1}_{\{\theta(k+1)=i\}} \mid r(k)=\ell, \theta(k)=j\right\} P[r(k)=\ell, \theta(k)=j] \\
= & \sum_{\ell \in \mathcal{N}} \sum_{j \in \mathcal{N}} E\left\{\left(\hat{A}_{j \ell} x_{k}+G_{j} w_{k}\right)\left(\hat{A}_{j \ell} x_{k}+G_{j} w_{k}\right)^{\prime}\right\} P[r(k)=\ell \mid \theta(k)=j] \times \\
& P[\theta(k+1)=i, \theta(k)=j] \\
= & \sum_{\ell \in \mathcal{N}} \sum_{j \in \mathcal{N}}\left(\hat{A}_{j \ell} E\left\{x_{k} x_{k}^{\prime} \mathbb{1}_{\{\theta(k)=j\}}\right\} \hat{A}_{j \ell}^{\prime}+G_{j} E\left\{w_{k} w_{k}^{\prime} \mathbb{1}_{\{\theta(k)=j\}}\right\} G_{j}^{\prime}\right) \hat{p}_{j \ell} p_{j i} \\
= & \sum_{\ell \in \mathcal{N}} \sum_{j \in \mathcal{N}} p_{j i} \hat{p}_{j \ell}\left(\hat{A}_{j \ell} X_{j}(k) \hat{A}_{j \ell}^{\prime}+G_{j} \pi_{j}(k) G_{j}^{\prime}\right) \\
= & \hat{\mathfrak{T}}_{\hat{A}, i}(X(k))+\hat{\Sigma}_{i}(k) .
\end{aligned}
$$


O resultado a seguir apresenta a formulação do problema do custo de T estágios com observação indireta da variável $\theta(k), k \in \mathcal{K}$.

Lema 5.2. Considere o SLSM $\hat{\mathbf{\Phi}}$ definido na Equação 5.5. Então, o custo de $T$ estágios associado à coleção de sequências de ganhos $\mathbf{F}=\left\{\mathbf{F}_{\mathbf{r}(\mathbf{k})}, k \in \mathcal{K}\right\}$ em cada nível de observação, $c \in\left[\frac{1}{N}, 1\right]$, é dado por

$$
\hat{\mathrm{J}}_{T, c}=\sum_{k=0}^{T-1}\left(\langle X(k), C\rangle+\sum_{\ell=1}^{N}\langle X(k), \hat{\mathrm{Q}}\rangle\right),
$$

sendo que $\hat{\mathcal{Q}} \in \mathbb{S}^{r+}$ com $\hat{Q}_{i}=\hat{p}_{i \ell} F_{\ell}(k)^{\prime} D_{i} F_{\ell}(k)$ para todo $i, \ell \in \mathcal{N}$.

Demonstração. Considere o custo por estágio $\hat{J}_{k, c}$ para o sistema $\hat{\boldsymbol{\Phi}}$ associado ao nível de observação $c$, dado por

$$
\begin{aligned}
\hat{J}_{k, c} & =E\left\{y_{k}\right\}=E\left\{x_{k}^{\prime} \hat{C}_{\theta(k), r(k)} x_{k}\right\} \\
& =E\left\{x_{k}^{\prime} C_{\theta(k)} x_{k}\right\}+E\left\{x_{k}^{\prime} F_{r(k)}(k)^{\prime} D_{\theta(k)} F_{r(k)}(k) x_{k}\right\} .
\end{aligned}
$$

Pela Proposição 2.1 obtém-se que

$$
E\left\{x_{k}^{\prime} C_{\theta(k)} x_{k}\right\}=\langle X(k), C\rangle, \forall k \in \mathcal{K} .
$$

Para o segundo termo, tem-se que

$$
\begin{aligned}
& \sum_{\ell \in \mathcal{N}} \sum_{i \in \mathcal{N}} E\left\{x_{k}^{\prime} F_{r(k)}(k)^{\prime} D_{\theta(k)} F_{r(k)}(k) x_{k} \mathbb{1}_{\{\theta(k)=i, r(k)=\ell\}}\right\}= \\
= & \sum_{\ell \in \mathcal{N}} \sum_{i \in \mathcal{N}} E\left\{x_{k}^{\prime} F_{r(k)}(k)^{\prime} D_{\theta(k)} F_{r(k)}(k) x_{k} \mid \theta(k)=i, r(k)=\ell\right\} P[r(k)=\ell, \theta(k)=i] \\
= & \sum_{\ell \in \mathcal{N}} \sum_{i \in \mathcal{N}} E\left\{x_{k}^{\prime} F_{r(k)}(k)^{\prime} D_{\theta(k)} F_{r(k)}(k) x_{k} \mid \theta(k)=i, r(k)=\ell\right\} P[r(k)=\ell \mid \theta(k)=i] \times \\
& P[\theta(k)=i] \\
= & \sum_{\ell \in \mathcal{N}} \sum_{i \in \mathcal{N}} E\left\{x_{k}^{\prime} F_{r(k)}(k)^{\prime} D_{\theta(k)} F_{r(k)}(k) x_{k} \mathbb{1}_{\{\theta(k)=i\}} \mid r(k)=\ell\right\} \hat{p}_{i \ell} \\
= & \sum_{\ell \in \mathcal{N}} \sum_{i \in \mathcal{N}} E\left\{\operatorname{Tr}\left\{F_{\ell}(k)^{\prime} D_{\theta(k)} F_{\ell}(k) x_{k} x_{k}^{\prime}\right\} \mathbb{1}_{\{\theta(k)=i\}}\right\} \hat{p}_{i \ell} \\
= & \sum_{\ell \in \mathcal{N}} \sum_{i \in \mathcal{N}} \operatorname{Tr}\left\{F_{\ell}(k)^{\prime} D_{\theta(k)} F_{\ell}(k) E\left\{x_{k} x_{k}^{\prime} \mathbb{1}_{\{\theta(k)=i\}}\right\}\right\} \hat{p}_{i \ell} \\
= & \sum_{\ell \in \mathcal{N}} \sum_{i \in \mathcal{N}} \operatorname{Tr}\left\{X_{i}(k) \hat{p}_{i \ell} F_{\ell}(k)^{\prime} D_{i} F_{\ell}(k)\right\} .
\end{aligned}
$$

Considere a sequência de matrizes $\hat{Q}_{i}=\hat{p}_{i \ell} F_{\ell}(k)^{\prime} D_{i} F_{\ell}(k)$ para todo $i, \ell \in \mathcal{N}$. Então, pela Proposição 2.1 tem-se que

$$
E\left\{x_{k}^{\prime} F_{r(k)}(k)^{\prime} D_{\theta(k)} F_{r(k)}(k) x_{k}\right\}=\sum_{\ell=1}^{N}\langle X(k), \hat{\mathcal{Q}}\rangle .
$$


Portanto,

$$
\begin{aligned}
\hat{\jmath}_{T, c} & =\sum_{k=0}^{T-1} E\left\{y_{k}\right\}=\sum_{k=0}^{T-1} \hat{J}_{k, c} \\
& =\sum_{k=0}^{T-1}\left(\langle X(k), C\rangle+\sum_{\ell=1}^{N}\langle X(k), \hat{Q}\rangle\right) .
\end{aligned}
$$

A partir destes resultados, pode-se introduzir o problema de otimização, que consiste em determinar uma coleção de sequência de ganhos $\mathbf{F} \in \mathcal{F}$, que minimize o índice de desempenho em termos do valor esperado. Formalmente, tem-se que

$$
\begin{aligned}
& \min _{\mathbf{F} \in \mathcal{F}} \hat{\mathcal{J}}_{T, c}=\sum_{k=0}^{T-1} \hat{J}_{k, c} \\
& \text { s.a. }\left\{\begin{array}{l}
X_{i}(k+1)=\hat{\mathcal{T}}_{\hat{A}}(X(k-1))+\hat{\Sigma}(k-1), \quad k \geq 0, \\
X_{i}(0)=\pi_{i}(0) x_{0} x_{0}^{\prime}, \quad i \in \mathcal{N} .
\end{array}\right.
\end{aligned}
$$

No Algoritmo 5.1 é apresentada a estratégia de observação indireta da variável $\theta(k), \forall k \in \mathcal{K}$, para o problema do custo de T estágios em cada nível de observação. Este método gera uma sequência de níveis de observação, $c^{(\eta)}$, que inicia $\operatorname{com} c^{(0)}=1$ e decresce a uma taxa $t$ até alcançar o nível $c=\frac{1}{N}$. Para $c=1$ tem-se o cenário de observação completa cuja solução é obtida pela ERR (COSTA et al., 2005). Logo, pode-se inicializar o algoritmo com a sequência de ganhos dada pela ERR (Apêndice B).

Algoritmo 5.1 Estratégia de Observação Indireta

Passo 0: Inicie o processo em $\eta=0$. Faça $c^{(\eta)}=1$ e a coleção de sequências de ganhos $\mathbf{F}^{(\eta)}$ é tal que $F_{\ell}(k)=g_{\ell}$ para todo $k \in \mathcal{K}$ sendo que $g_{\ell}, \ell \in \mathcal{N}$, é a solução obtida pela ERR.

Passo 1: Determine o ganho ótimo $\mathbf{K}^{*}$ que minimiza o custo $\hat{\partial}_{T, c}$, a partir do ganho inicial dado por $\mathbf{F}^{(\eta)}$.

Passo 2: Faça $\eta=\eta+1$. Seja $c^{(\eta)}=c^{(\eta-1)}-t^{(\eta)}$.

Passo 3: Faça $\mathbf{F}^{(\eta)}=\mathbf{K}^{*}$ e volte para o passo 1 .

Critério de Parada: $c^{(\eta)}=\frac{1}{N}$. 
Neste trabalho, considera-se $t^{(\eta)}=0,1$ para todo $\eta$ no passo 2, mas acreditamos que uma estratégia com $t^{(\eta)}$ variando, como no Algoritmo 5.3, possa obter melhores resultados. Além disso, no passo 1 pode-se utilizar uma adaptação do método variacional (Capítulo 3) ou do método de Newton (Capítulo 4) para obter um resultado que pode ser uma aproximação para a solução do problema do custo de $\mathrm{T}$ estágios com observação indireta da variável $\theta$.

Na Seção 5.2 é apresentada uma formulação que permite utilizar o método variacional, com a cadeia de Markov original, para o problema do custo de T estágios com observação indireta da variável $\theta(k)$ e controle dado pela Equação 5.4. O método de direção de busca é facilmente estendido para a utilização desta estratégia e, portanto, será omitido.

\subsection{Método Variacional Adaptado para a Observação Parcial}

Os resultados a seguir apresentam uma condição de otimalidade para o problema do custo de $T$ estágios em cada cada nível de observação $c \in\left[\frac{1}{N}, 1\right]$, representado na Equação 5.11. Para a coleção de sequências de ganhos $\mathbf{F} \in \mathcal{F}$ define-se o funcional

$$
W\left(x_{k}, \theta(k)\right)=E\left\{\sum_{t=k}^{T-1} y_{t} \mid x_{k}, \theta(k)\right\},
$$

sendo que $W\left(x_{T}, \theta(T)\right)=0$. Este funcional é uma adaptação de Vargas (2004) que apresenta uma expressão determinística equivalente ao custo $\hat{\partial}_{T, c}(\mathbf{F})$.

Lema 5.3. Considere os operadores $\mathscr{E}, \mathcal{L}: \mathbb{S}^{r 0} \rightarrow \mathbb{S}^{r 0}$ como na Definição 2.3. Então, definem-se $\hat{L}(k) \in \mathbb{S}^{r 0}$ e $\hat{\omega}(k) \in \mathbb{M}^{1}$, para cada $k=0, \ldots, T-1$, tais que:

$$
\begin{gathered}
\hat{L}_{i}(k)=\sum_{\ell=1}^{N}\left(C_{i}+F_{\ell}(k)^{\prime} D_{i} F_{\ell}(k)+\mathcal{L}_{\hat{A}}(\hat{L}(k+1))\right) \hat{p}_{i \ell}, \\
\hat{\omega}_{i}(k)=\sum_{\ell=1}^{N}\left(\mathscr{E}(\hat{\omega}(k+1))+\operatorname{Tr}\left\{\mathscr{E}(\hat{L}(k+1)) G_{i} G_{i}^{\prime}\right\}\right) \hat{p}_{i \ell},
\end{gathered}
$$

sendo que $\hat{L}_{i}(T)=0$ e $\hat{\omega}_{i}(T)=0$, para todo $i \in \mathcal{N}$.

Então, o funcional (5.12) é equivalente a

$$
W\left(x_{k}, \theta(k)\right)=x_{k}^{\prime} \hat{L}_{\theta(k)}(k) x_{k}+\hat{\omega}_{\theta(k)}(k) .
$$


Demonstração. A prova será feita por indução sobre $k \in \mathcal{K}$. Seja $k=T$, então pela Equação 5.12 e a condição final $\hat{L}_{\theta(T)}(T)=0$ tem-se que $W\left(x_{T}, \theta(T)\right)=x_{T}^{\prime} L_{\theta(T)}(T) x_{T}=$ 0 . Assim, o resultado é válido para $k=T$.

Suponha que a Equação 5.15 seja válida para $k+1$,

$$
W\left(x_{k+1}, \theta(k+1)=j\right)=x_{k+1}^{\prime} \hat{L}_{j}(k+1) x_{k+1}+\hat{\omega}_{j}(k+1) .
$$

Agora, deve-se mostrar que o Equação 5.15 é válida para $k$. Note que o funcional $W\left(x_{k}, \theta(k)\right)$ pode ser reescrito da seguinte forma:

$$
\begin{aligned}
W\left(x_{k}, \theta(k)\right) & =\sum_{\ell=1}^{N} E\left\{\sum_{t=k}^{T-1} y_{t} \mathbb{1}_{\{r(k)=\ell\}} \mid x_{k}, \theta(k)=i\right\} \\
& =\sum_{\ell=1}^{N}\left(y_{k}+E\left\{W\left(x_{k+1}, \theta(k+1)=j\right) \mid x_{k}, \theta(k)=i\right\}\right) \hat{p}_{i \ell} .
\end{aligned}
$$

então, segue que

$$
\begin{aligned}
W\left(x_{k}, \theta(k)\right)= & \sum_{\ell=1}^{N}\left(y_{k}+E\left\{W\left(x_{k+1}, \theta(k+1)\right) \mid x_{k}, \theta(k)=i\right\}\right) \hat{p}_{i \ell} \\
= & \sum_{\ell=1}^{N}\left(y_{k}+E\left\{x_{k+1}^{\prime} \hat{L}_{\theta(k+1)}(k+1) x_{k+1}+\hat{\omega}_{\theta(k+1)}(k+1) \mid x_{k}, \theta(k)=i\right\}\right) \hat{p}_{i \ell} \\
= & \sum_{\ell=1}^{N}\left(\left(x_{k}^{\prime} C_{i} x_{k}+u_{k}^{\prime} D_{i} u_{k}\right)+E\left\{x_{k+1}^{\prime} \hat{L}_{\theta(k+1)}(k+1) x_{k+1}+\right.\right. \\
& \left.\left.\hat{\omega}_{\theta(k+1)}(k+1) \mid x_{k}, \theta(k)=i\right\}\right) \hat{p}_{i \ell,}
\end{aligned}
$$

usando o fato de que $u_{k}=F_{\ell}(k) x_{k}$ e $\hat{A}_{i, \ell}=A_{i}+B_{i} F_{\ell}(k)$ tem-se que,

$$
\begin{aligned}
W\left(x_{k}, \theta(k)\right)= & \sum_{\ell=1}^{N}\left(\left(x_{k}^{\prime} C_{i} x_{k}+x_{k}^{\prime} F_{\ell}^{\prime}(k) D_{i} F_{\ell}(k) x_{k}\right)+x_{k}^{\prime} \hat{A}_{i, \ell}^{\prime} \times\right. \\
& E\left\{\hat{L}_{\theta(k+1)}(k+1) \mid x_{k}, \theta(k)=i\right\} \hat{A}_{i, \ell} x_{k}+ \\
& E\left\{w_{k}^{\prime} G_{i}^{\prime} \hat{L}_{\theta(k+1)}(k+1) G_{i} w_{k} \mid x_{k}, \theta(k)=i\right\}+ \\
& \left.E\left\{\hat{\omega}_{\theta(k+1)}(k+1) \mid x_{k}, \theta(k)=i\right\}\right) \hat{p}_{i \ell .}
\end{aligned}
$$


Note que,

$$
\begin{aligned}
E\left\{\hat{L}_{\theta(k+1)}(k+1) \mid x_{k}, \theta(k)=i\right\}= & \sum_{j=1}^{N} E\left\{\hat{L}_{\theta(k+1)}(k+1) \mid \theta(k)=i, \theta(k+1)=j\right\} \\
& P[\theta(k+1)=j \mid \theta(k)=i] \\
= & \sum_{j=1}^{N} p_{i j} \hat{L}_{j}(k+1)=\mathscr{E}_{i}\left(\hat{L}_{k+1}\right) . \\
E\left\{\hat{\omega}_{\theta(k+1)}(k+1) \mid x_{k}, \theta(k)=i\right\}= & \sum_{j=1}^{N} E\left\{\hat{\omega}_{\theta(k+1)}(k+1) \mid \theta(k)=i, \theta(k+1)=j\right\} \\
& P[\theta(k+1)=j \mid \theta(k)=i] \\
= & \sum_{j=1}^{N} p_{i j} \hat{\omega}_{j}=\mathscr{E}(\hat{\omega}(k+1)) .
\end{aligned}
$$

$E\left\{w_{k}^{\prime} G_{i}^{\prime} \hat{L}_{\theta(k+1)}(k+1) G_{i} w_{k} \mid x_{k}, \theta(k)=i\right\}=$

$=E\left\{\operatorname{Tr}\left\{\hat{L}_{\theta(k+1)}(k+1) G_{i} w_{k} w_{k}^{\prime} G_{i}^{\prime}\right\} \mid \theta(k)=i\right\}$

$=\operatorname{Tr}\left\{E\left\{\hat{L}_{\theta(k+1)}(k+1) G_{i} w_{k} w_{k}^{\prime} G_{i}^{\prime}\right\} \mid \theta(k)=i\right\}$

$=\operatorname{Tr}\left\{\mathscr{E}_{i}(\hat{L}(k+1)) G_{i} G_{i}^{\prime}\right\}$.

Finalmente, tem-se que

$$
\begin{aligned}
W\left(x_{k}, \theta(k)\right)= & \sum_{\ell=1}^{N}\left(x_{k}^{\prime}\left\{C_{i}+F_{\ell}^{\prime}(k) D_{i} F_{\ell}(k)+\hat{A}_{i, \ell}^{\prime} \mathscr{E}_{i}(\hat{L}(k+1)) \hat{A}_{i, \ell}\right\} x_{k}+\right. \\
& \left.\operatorname{Tr}\left[\mathscr{E}_{i}(\hat{L}(k+1)) G_{i} G_{i}^{\prime}\right]+\mathscr{E}_{i}(\hat{\omega}(k+1))\right) \hat{p}_{i \ell} \\
= & x_{k}^{\prime} \sum_{\ell=1}^{N}\left(C_{i}+F_{\ell}^{\prime}(k) D_{i} F_{\ell}(k)+\hat{A}_{i, \ell}^{\prime} \mathscr{E}_{i}(\hat{L}(k+1)) \hat{A}_{i, \ell}\right) \hat{p}_{i \ell} x_{k}+ \\
& \sum_{\ell=1}^{N}\left(\mathscr{E}_{i}(\hat{\omega}(k+1))+\operatorname{Tr}\left[\mathscr{E}_{i}(\hat{L}(k+1)) G_{i} G_{i}^{\prime}\right]\right) \hat{p}_{i \ell} \\
= & x_{k}^{\prime} \hat{L}_{i}(k) x_{k}+\hat{\omega}_{i}(k) .
\end{aligned}
$$

O Lema 5.3 fornece uma formulação essencialmente idêntica ao método variacional (Capítulo 3), com exceção da sequência de matrizes $L \in \mathbb{S}^{r 0}$ e do escalar $\omega(k) \in \mathbb{M}^{1}$ 
que são alterados. Isto permite estender o resultado do Teorema 3.1, como segue. A demonstração será omitida.

Lema 5.4. Suponha que a coleção de sequências de ganhos $\mathbf{F} \in \mathcal{F}$ realiza o mínimo global do problema do custo de $T$ estágios. Então cada sequência de ganhos $\mathbf{F}_{\ell}, \ell \in \mathcal{N}$, satisfaz, para cada $k \in \mathcal{K}$, a equação

$$
\sum_{i=1}^{N}\left[\left(D_{i}+B_{i}^{\prime} \mathscr{E}_{i}(\hat{L}(k+1)) B_{i}\right) F_{\ell}(k)+B_{i}^{\prime} \mathscr{E}_{i}(\hat{L}(k+1)) A_{i}\right] X_{i}(k)=0 .
$$

No Algoritmo 5.2 é apresentado o método variacional adaptado para a observação parcial que resulta em uma coleção de sequências de ganhos $\mathbf{F}_{c}$ que minimiza localmente o custo por $T$ estágios, $\mathcal{J}_{T, c}$ em cada nível de observação $c \in\left[\frac{1}{N}, 1\right]$, satisfazendo a condição de otimalidade (Equação 5.16).

Algoritmo 5.2 Método Variacional Adaptado para a Observação Parcial

Passo 0: Inicie o processo em $\eta=0$.

Escolha uma coleção de sequências inicial de ganhos $\mathbf{F}_{c}^{(\eta)}$.

Passo 1: Para todo $k \in \mathcal{K}$ encontre $X^{(\eta)}(k) \in \mathcal{S}^{r 0}$ tal que

$$
\left\{\begin{array}{l}
X^{(\eta)}(k+1)=\hat{\mathcal{T}}_{\bar{A}}\left(X^{(\eta)}(k)\right)+\hat{\Sigma}(k) \\
X_{i}^{(\eta)}(0)=\pi_{i}(0) x_{0} x_{0}^{\prime}, \forall i \in \mathcal{N}
\end{array}\right.
$$

onde os operadores $\hat{\mathcal{T}}$ e $\hat{\Sigma}$ são dados pela Definição 5.1 .

Passo 2: Faça $\eta=\eta+1$.

Para cada sequência de ganhos $\mathbf{F}_{\ell}{ }^{(\eta)}, \ell \in \mathcal{N}$, e para cada $k=T-1, T-2, \ldots, 0$, determine $F_{\ell}^{(\eta)}(k)$ resolvendo a Equação 5.16 e calcule $\hat{L}^{(\eta)} \in \mathcal{S}^{r 0}$ pela Equação 5.13 .

Critério de Parada: $\left|\mathcal{J}_{T, c}\left(\mathbf{F}_{c}^{(\eta)}\right)-\mathcal{J}_{T, c}\left(\mathbf{F}_{c}^{(\eta-1)}\right)\right| / \mathcal{J}_{T, c}\left(\mathbf{F}_{c}^{(\eta)}\right)<\epsilon$, para $\epsilon$ dado. Se o critério não for satisfeito, volte para o passo 2.

\subsection{Estratégia de Observação Indireta para o CMLP}

Considere o SLSM $\hat{\Phi}$ (Equação 5.5)

$$
\hat{\mathbf{\Phi}}: \begin{cases}x_{k+1}=\hat{A}_{\theta(k), r(k)} x_{k}+G_{\theta(k)} w_{k}, & \forall k \geq 0, \\ y_{k}=x_{k}^{\prime} \hat{C}_{\theta(k), r(k)} x_{k}, & \theta(0) \sim \pi(0),\end{cases}
$$


sendo que $\hat{A}_{\theta(k), r(k)}=A_{\theta(k)}+B_{\theta(k)} F_{r(k)}(k)$ e $\hat{C}_{\theta(k), r(k)}=C_{\theta(k)}+F_{r(k)}(k)^{\prime} D_{\theta(k)} F_{r(k)}(k)$. Além disso, considera-se que a cadeia de Markov $\Theta$ é ergódica (veja Observação 2.2). Define-se o CMLP associado a cada nível de observação $c \in\left[\frac{1}{N}, 1\right]$, por

$$
\hat{J}_{c}=\limsup _{T \rightarrow \infty} \frac{\hat{\jmath}_{T, c}}{T}
$$

Os resultados a seguir apresentam uma formulação para o problema do CMLP com observação indireta da variável $\theta(k)$ e controle dado pela Equação 5.4.

Definição 5.2. (VARGAS et al., 2006, Def. 2) O sistema $\mathbf{\Phi}$ (Equação 5.1) é estabilizável na média quadrática (MS-estabilizável) se existe uma coleção de sequência ganhos $\mathbf{F} \in \mathcal{F}$ tal que o raio espectral do operador $\hat{\mathcal{T}}_{\hat{A}}$ é menor que um. Neste caso, diz-se que $\mathbf{F} \in \mathcal{F}$ é MS-estabilizável.

A Proposição 5.1 e o Corolário 5.1 são uma adaptação da Proposição 2.2 e do Corolário 2.1 para o problema de observação indireta da variável $\theta(k)$. As demonstrações são análogas ao problema original, ou seja, sem observação da variável $\theta(k)$ e podem ser encontradas em Costa et al. (2005) e Vargas et al. (2006), respectivamente.

Proposição 5.1. Suponha que $\mathbf{F} \in \mathcal{F}$ é MS-estabilizável. Então existe um único $X \in \mathcal{S}^{r 0}$ que satisfaz $X=\hat{\mathfrak{T}}_{\hat{A}}(X)+\hat{\Sigma}$, ou equivalentemente

$$
X=\lim _{k \rightarrow \infty} X(k) \text { e } \hat{\Sigma}=\lim _{k \rightarrow \infty} \hat{\Sigma}(k),
$$

sendo que as matrizes $\hat{\mathcal{T}}_{\hat{A}}$ e $\hat{\Sigma}(k)$ dependem do nível de observação $c \in\left[\frac{1}{N}, 1\right]$. Além disso, tem-se que $X=\sum_{k=0}^{\infty} \hat{\mathcal{T}}_{\hat{A}}^{(k)}(\hat{\Sigma})$.

Corolário 5.1. Suponha que $\Theta$ é ergódica e seja $F=\left\{F_{1}, \ldots, F_{N}\right\} \in \mathbf{F}$ uma sequência ganhos MS-estabilizável no nível de observação $c \in\left[\frac{1}{N}, 1\right]$. Então,

$$
\hat{J}_{c}=\langle X, C\rangle+\sum_{\ell=1}^{N}\langle X, \hat{\mathcal{Q}}\rangle \text {, }
$$

sendo que $X=\sum_{k=0}^{\infty} \hat{\mathfrak{T}}_{\hat{A}}^{(k)}(\hat{\Sigma})$ e $\hat{Q}_{i}=\hat{p}_{i \ell} F_{\ell}^{\prime} D_{i} F_{\ell} \in \mathcal{S}^{r+}$ para todo $i, \ell \in \mathcal{N}$.

Logo, pelos resultados obtidos, pode-se formular o problema de otimização do CMLP com observação indireta da variável $\theta(k)$, que consiste em determinar uma sequência de ganhos $F=\left\{F_{1}, \ldots, F_{N}\right\} \in \mathbf{F}$ que minimize o custo $\hat{J}_{c}$ para cada nível de observação $c$. Formalmente, tem-se que 


$$
\begin{aligned}
& \min _{F \in \mathbf{F}} \hat{J}_{c}=\langle X, C\rangle+\sum_{\ell=1}^{N}\langle X, \hat{\mathcal{Q}}\rangle \\
& \text { s.a. }\left\{\begin{array}{l}
X=\hat{\mathfrak{T}}_{\hat{A}}(X)+\hat{\Sigma}, \\
F \text { é estabilizante, }
\end{array}\right.
\end{aligned}
$$

sendo que $c \in\left[\frac{1}{N}, 1\right], \hat{Q}_{i}=\hat{p}_{i \ell} F_{\ell}^{\prime} D_{i} F_{\ell} \in \mathcal{S}^{r+}$ para todo $i, \ell \in \mathcal{N}$.

O Lema 5.5 apresenta uma fórmula que permite obter uma condição para a MSestabilidade em termos dos autovalores de uma matriz $\mathcal{A} \in \mathcal{M}^{r r}$ para cada nível de observação $c \in\left[\frac{1}{N}, 1\right]$.

Lema 5.5. Seja $X(k) \in \mathbb{S}^{r 0}$ definido no Lema 5.1, então

$$
\operatorname{vec}(X(k+1))=\mathcal{A}_{c} \operatorname{vec}(X(k))+\operatorname{vec}(\hat{\Sigma}),
$$

sendo que $\mathcal{A}_{c} \in \mathcal{M}^{r r}$ é definido por $\mathcal{A}_{c}=\left[a_{i j}\right], i, j \in \mathcal{N}$ com

$$
a_{i j}=\sum_{\ell=1}^{N} p_{j i} \hat{p}_{j \ell}\left(\hat{A}_{j \ell} \otimes \hat{A}_{j \ell}\right) .
$$

Demonstração. Considere $X_{i}(k+1)=\hat{\mathcal{T}}_{\hat{A}, i}(X(k))+\hat{\Sigma}_{i}(k)$. Suponha que $\hat{\Sigma}_{i}(k)=0$ para todo $i \in \mathcal{N}$ e $k \in \mathcal{K}$ (a prova para o caso em que $\hat{\Sigma}(k) \neq 0$ é análoga). Tem-se que

$$
X_{i}(k+1)=\sum_{\ell \in \mathcal{N}} \sum_{j \in \mathcal{N}} p_{j i} \hat{p}_{j \ell} \hat{A}_{j \ell} X_{j}(k) \hat{A}_{j \ell}^{\prime} .
$$

Aplicando o produto de Kronecker em ambos os lados da equação, obtém-se que

$$
\operatorname{vec}\left(X_{i}(k+1)\right)=\sum_{\ell \in \mathcal{N}} \sum_{j \in \mathcal{N}} p_{j i} \hat{p}_{j \ell}\left(\hat{A}_{j \ell} \otimes \hat{A}_{j \ell}^{\prime}\right) \operatorname{vec}\left(X_{j}(k)\right) .
$$

O que resulta em

$$
\operatorname{vec}\left(X_{i}(k+1)\right)=\sum_{j \in \mathcal{N}} a_{i j} \operatorname{vec}\left(X_{j}(k)\right)
$$

Lema 5.6. Uma coleção de sequências de ganhos $\mathbf{F} \in \mathcal{F}$ é $M S$-estabilizável se e somente se raio espectral da matriz $\mathcal{A}$ é menor que um.

Lema 5.7. Se a sequência de ganhos $\mathbf{F} \in \mathcal{F}$ é $M S$-estabilizável em um nível de observação $c$, então existe um escalar $\delta>0$ tal que a coleção de sequências de ganhos $\mathbf{K}$ MS-estabilizável no nível de observação $c-\delta$. 
Demonstração. O sistema $\Phi$ (Equação 5.1) é estável com controle dado por $u_{k}=$ $F_{r(k)} x_{k}$ se e somente se o raio espectral da matriz $\mathcal{A}_{c}$ no nível de observação $c$ é menor que um. Os autovalores de $\mathcal{A}$ dependem continuamente de $c$, de modo que existe um escalar $\delta>0$ tal que o raio espectral da matriz $\mathcal{A}_{c-\delta}$ também é menor que um. Isto implica que existe um ganho K MS-estabilizável no nível de observação $c-\delta$.

No Algoritmo 5.3 é apresentada a estratégia de observação indireta da variável $\theta(k), \forall k \in \mathcal{K}$, para o problema do CMLP em cada nível de observação $c$. No passo 1 do algoritmo pode-se utilizar o método variacional adaptado (Seção 5.2) ou o método de direção de busca adaptado para obter um resultado que pode ser uma aproximação para a solução do problema do CMLP com observação indireta da variável $\theta$.

Algoritmo 5.3 Estratégia de Observação Indireta para o problema do CMLP

Passo 0: Inicie o processo em $\eta=0$. Faça $c^{(\eta)}=1$ e a coleção de ganhos $F^{(\eta)}$ é tal que $F_{\ell}=g_{\ell}$, sendo que $g_{\ell}, \ell \in \mathcal{N}$, é a solução estabilizante da EARA. Se não existir a solução estabilizante para a EARA, então PARE.

Passo 1: Determine o ganho ótimo $K^{*}$ que minimiza o custo $\hat{J}_{c^{(\eta)}}$, a partir do ganho inicial dado por $F^{(\eta)}$.

Passo 2: Faça $\eta=\eta+1$. Seja $c^{(\eta)}=c^{(\eta-1)}-t^{(\eta)} \operatorname{com} t^{(\eta)} \in\left(0, c^{(\eta-1)}-\frac{1}{N}\right]$.

Passo 3: Faça $F^{(\eta)}=K^{*}$. Se $F^{(\eta)}$ é MS-estabilizável então volte para o passo 1.

Caso contrário, faça $t^{(\eta-1)}=t^{(\eta-1)} / 2$, determine $c^{(\eta)}=c^{(\eta-1)}-t^{(\eta-1)}$ e volte para o passo 3 .

Critério de Parada: Se $c^{(\eta)}=\frac{1}{N}$ ou se $c^{(\eta)}-c^{(\eta-1)}<\epsilon$ para $\epsilon$ dado. Se o critério não for satisfeito, volte para o passo 3 .

\subsection{Exemplos Numéricos}

Nesta seção são apresentados exemplos numéricos para ilustrar a utilização da estratégia de observação indireta da variável $\theta(k)$ cujo resultado pode ser uma aproximação para a solução do problema do custo de T estágios (Equação 2.26).

Exemplo 5.1. Considere os seguintes parâmetros para o SLSM $\Phi$ (Equação 2.11),

$$
A_{1}=\left[\begin{array}{rrr}
0,9537 & -0,1622 & 0,4359 \\
-0,3021 & 0,0575 & -0,1256 \\
-1,3963 & -0,0777 & -0,5177
\end{array}\right], A_{2}=\left[\begin{array}{rrr}
0,6161 & -0,1121 & 0,0423 \\
-0,0332 & 0,5068 & 0,0480 \\
-0,0645 & -0,2783 & 0,7335
\end{array}\right]
$$




$$
\begin{aligned}
& C_{1}=\left[\begin{array}{lll}
0,5041 & 0,0275 & 0,0135 \\
0,0275 & 0,0015 & 0,0007 \\
0,0135 & 0,0007 & 0,0004
\end{array}\right], C_{2}=\left[\begin{array}{rrr}
1,6707 & -3,4086 & -0,8473 \\
-3,4086 & 6,9542 & 1,7286 \\
-0,8473 & 1,7286 & 0,4297
\end{array}\right] \\
& B_{1}=\left[\begin{array}{r}
0,3574 \\
1,0266 \\
-1,4121
\end{array}\right], B_{2}=\left[\begin{array}{r}
-2,7909 \\
-1,2206 \\
-0,1844
\end{array}\right], G_{1}=\left[\begin{array}{r}
3,1349 \\
0,3105 \\
-2,8103
\end{array}\right], G_{2}=\left[\begin{array}{l}
0,8341 \\
0,7454 \\
0,8203
\end{array}\right] \text {, } \\
& \mathbb{P}=\left[\begin{array}{ll}
0,5556 & 0,4444 \\
0,9935 & 0,0065
\end{array}\right], \pi(0)=\left[\begin{array}{ll}
0,2016 & 0,7984
\end{array}\right], x_{0}=\left[\begin{array}{r}
3,4578 \\
-1,2781 \\
1,0086
\end{array}\right], \\
& D_{1}=0,0630 \text { e } D_{2}=0,1832 .
\end{aligned}
$$

O Algoritmo 5.1 foi implementado no software $M A T L A B^{\circledR}$, sendo que no passo 1 foi utilizado o método variacional adaptado para a observação parcial (Seção 5.2) com tolerância $\epsilon=10^{-4}$ e horizonte $T=50$.

$\mathrm{O}$ custo de $\mathrm{T}$ estágios para o nível de observação $c^{(5)}=\frac{1}{2}$ obtido foi igual a

$$
\mathcal{J}_{T, c^{(5)}}\left(\mathbf{F}^{*}\right) \approx 829,1125
$$

sendo que a coleção de sequências de ganhos $\mathbf{F}^{*}$ obtida está disponível no Apêndice D.

Na Figura 5.1 são apresentados os comportamentos da norma do segundo momento $X_{c}(T)$ em função do número de iterações realizadas pelo método variacional adaptado para a observação parcial, $\bar{\eta}$, e do custo de $\mathrm{T}$ estágios $\mathcal{J}_{T, c}$ em função do número de iterações $\eta$. Note que a sequência de pontos em todos os gráficos convergem conforme o número de iterações aumenta.

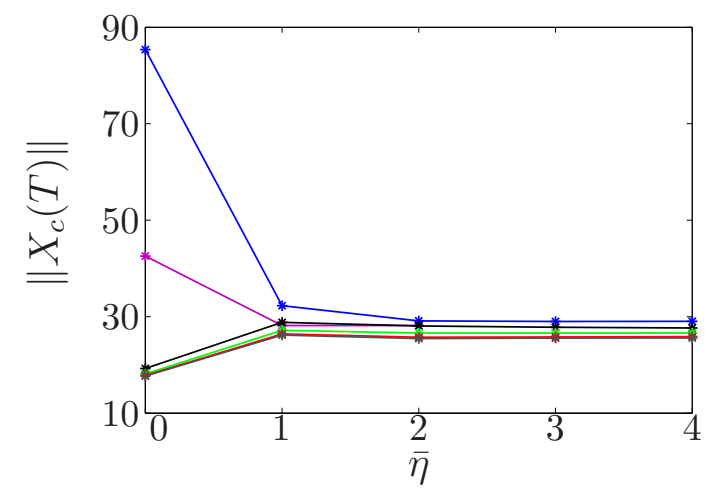

(a) Norma de $X_{c}(T)$ versus $\bar{\eta}$

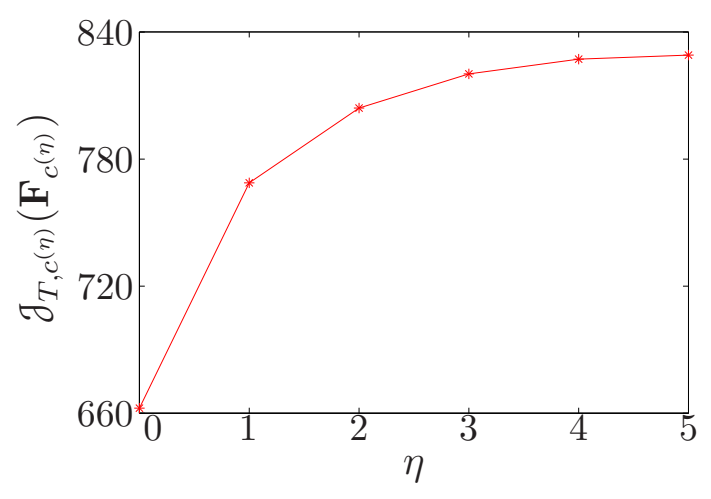

(b) Custo $\mathcal{J}_{T, c}(\eta)\left(\mathbf{F}_{c^{(\eta)}}\right)$ versus $\eta$

Figura 5.1: Resultados da estratégia de observação indireta para o Exemplo 5.1 
Exemplo 5.2. Considere os seguintes parâmetros para o SLSM $\Phi$ (Equação 2.11),

$$
\begin{aligned}
& A_{1}=\left[\begin{array}{rrr}
-0,0553 & 0,2582 & -0,0244 \\
-0,2186 & 0,0416 & -0,0689 \\
-0,1306 & 0,1651 & -0,1114
\end{array}\right], A_{2}=\left[\begin{array}{rrr}
0,6921 & -0,5154 & 0,3931 \\
-0,3454 & 0,2515 & 0,0191 \\
-0,2812 & 0,3718 & 0,5518
\end{array}\right], \\
& C_{1}=\left[\begin{array}{rrr}
0,0409 & -0,0025 & -0,0209 \\
-0,0025 & 0,0437 & -0,0536 \\
-0,0209 & -0,0536 & 0,0799
\end{array}\right], C_{2}=\left[\begin{array}{rrr}
2,2730 & -1,3297 & 1,0180 \\
-1,3297 & 1,7525 & -0,9484 \\
1,0180 & -0,9484 & 0,5837
\end{array}\right] \text {, } \\
& B_{1}=\left[\begin{array}{r}
-0,7108 \\
0,5049 \\
0,3430
\end{array}\right], B_{2}=\left[\begin{array}{r}
-0,8123 \\
-0,4644 \\
0,1125
\end{array}\right], G_{1}=\left[\begin{array}{r}
-0,3082 \\
0,8908 \\
1,3520
\end{array}\right], G_{2}=\left[\begin{array}{l}
0,4257 \\
1,3741 \\
0,0137
\end{array}\right] \text {, } \\
& \mathbb{P}=\left[\begin{array}{ll}
0,8897 & 0,1103 \\
0,4385 & 0,5615
\end{array}\right], \pi(0)=\left[\begin{array}{ll}
0,8327 & 0,1673
\end{array}\right], x_{0}=\left[\begin{array}{c}
0,0359 \\
-0,6275 \\
0,5354
\end{array}\right],
\end{aligned}
$$

$D_{1}=0,6791$ e $D_{2}=0,3955$.

O Algoritmo 5.1 foi implementado, sendo que no passo 1 foi utilizado o método de Newton adaptado para a observação parcial com tolerância $\epsilon=10^{-4}$ e horizonte $T=50$.

O custo de T estágios para o nível de observação $c^{(5)}=\frac{1}{2}$ obtido foi igual a

$$
\mathcal{J}_{T, c^{(5)}}\left(\mathbf{F}^{*}\right) \approx 20,8383
$$

sendo que a coleção de sequências de ganhos $\mathbf{F}^{*}$ obtida está disponível no Apêndice D.

Na Figura 5.2 são apresentados os comportamentos da norma do segundo momento $X_{c}(T)$ em função do número de iterações realizadas pelo método de direção de busca adaptado para a observação parcial, $\bar{\eta}$, e do custo de $T$ estágios $\mathcal{J}_{T, c}$ em função do número de iterações $\eta$. Note que a sequência de pontos em todos os gráficos convergem conforme o número de iterações aumenta. 


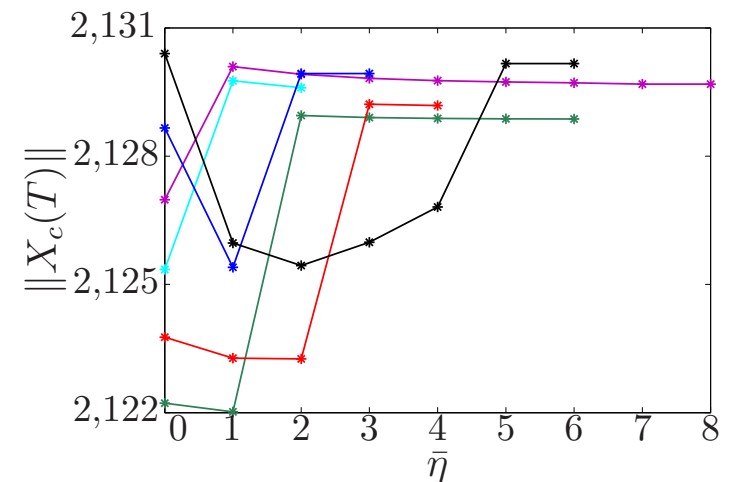

(a) Norma de $X_{c}(T)$ versus $\bar{\eta}$

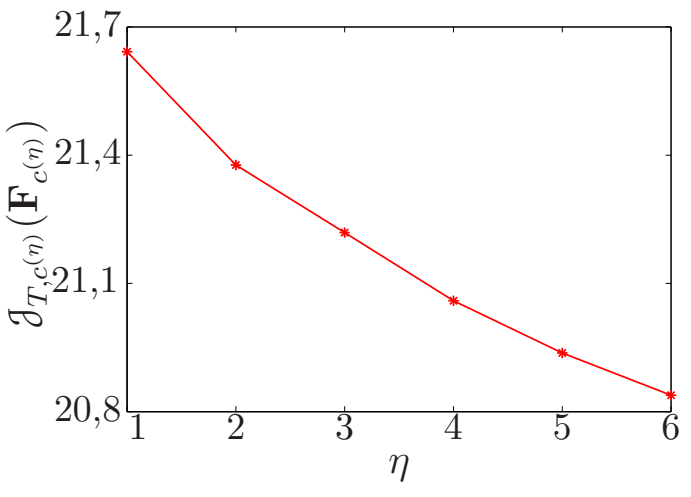

(b) Custo $\mathcal{J}_{T, c^{(\eta)}}\left(\mathbf{F}_{c^{(\eta)}}\right)$ versus $\eta$

Figura 5.2: Resultados da estratégia de observação indireta para o Exemplo 5.2 



\begin{tabular}{c|c|}
\cline { 2 - 2 } & Capítulo \\
\cline { 2 - 2 } & 6 \\
\hline
\end{tabular}

\section{Experimentos Computacionais}

Nos capítulos anteriores foram apresentados o método variacional (Capítulo 3), o método de direção de busca (Capítulo 4) e a estratégia de observação indireta (Capítulo 5), para o problema de otimização formulado na Equação 2.26, que consiste em determinar uma sequência de ganhos $\mathrm{g} \in \mathcal{G}$ que minimize o custo de $\mathrm{T}$ estágios, $\mathfrak{J}_{T}$, referente ao SLSM $\Phi$ (Equação 2.11). Neste capítulo são apresentados os experimentos computacionais realizados para avaliar e comparar o desempenho destes métodos. Na Seção 6.1 é proposto um Gerador de SLSM capaz de elaborar um conjunto de diversos exemplos numéricos de SLSM com diferentes características estruturais. Em Resultados Computacionais, Seção 6.2, é apresentada uma análise dos dados obtidos comparando os métodos desenvolvidos com o método variacional.

\subsection{Gerador de SLSM}

Considere o SLSM

$$
\hat{\mathbf{\Phi}}: \begin{cases}x_{k+1}=\hat{A}_{\theta(k)} x_{k}+\hat{B}_{\theta(k)} u_{k}+\hat{G}_{\theta(k)} w_{k}, & \forall k \geq 0, \\ y_{k}=x_{k}^{\prime} \hat{C}_{\theta(k)} x_{k}+u_{k}^{\prime} \hat{D}_{\theta(k)} u_{k}, & \theta(0) \sim \pi(0),\end{cases}
$$

com condição inicial $x_{0} \in \mathcal{M}^{r, 1}$, matriz de transição de probabilidades $\mathbb{P} \in \mathcal{M}^{N}$ e vetor de distribuição inicial $\pi(0) \in \mathcal{M}^{1, N}$.

A seguir, é feita uma descrição da construção dos elementos que compõem o SLSM $\hat{\boldsymbol{\Phi}}$ (Equação 6.1), sendo que este pode possuir certas propriedades da teoria de controle, tais como estabilidade, observabilidade e controlabilidade. 
Para gerar o SLSM, primeiramente é necessário estabelecer a quantidade de estados da cadeia, $N$, a qual será considerada como uma variável aleatória com distribuição equiprovável no intervalo $[a, b]$ (denota-se $N \sim \mathcal{E}(a, b)$ ), ou seja, tal que $P[N=x]=\frac{1}{b-a+1}$, sendo que $x$ é um número inteiro pertencente ao intervalo $[a, b]$. Em seguida, geram-se as sequências de matrizes do sistema. Na descrição da construção das matrizes, será considerado apenas um modo $i \in \mathcal{N}$ do sistema, pois a geração é idêntica para os demais.

Inicia-se com a determinação da dimensão da matriz $\tilde{A}$, a qual será dada por $r \sim \mathcal{E}(c, d)$. Considera-se a matriz $\tilde{A}$ na forma canônica de Jordan, pois esta estrutura viabiliza a análise da observabilidade, controlabilidade e estabilidade de cada modo do sistema. Dessa forma, tem-se que $\tilde{A}=\operatorname{diag}\left(J_{1}^{(i)}\left(\lambda_{1}, r_{1}\right), \ldots, J_{m}^{(i)}\left(\lambda_{m}, r_{m}\right)\right)$ onde cada bloco $J_{j}^{(i)}\left(\lambda_{j}, r_{j}\right), j=1, \ldots, m$, está associado ao autovalor $\lambda_{j}$ e tem dimensão igual a $r_{j}$ sendo que $\sum_{j=1}^{m} r_{j}=r$. Além disso, a parte real e imaginária de cada autovalor $\lambda_{j}$ têm distribuições uniformes no intervalo $(-1,1)$ e são independentes entre si.

As matrizes $\tilde{B}, \tilde{C}$ e $\tilde{G}$ são construídas de forma que os pares $(\tilde{A}, \tilde{B})$ e $(\tilde{A}, \tilde{G})$ podem ser controláveis, e o par $(\tilde{A}, \tilde{C})$ pode ser observável. Para determinar se cada modo $i$ do sistema terá ou não estas propriedades é feito um sorteio equiprovável. Para gerar estas matrizes são utilizados os resultados de Chen (1998) que estão apresentados no Apêndice C.

Por simplicidade, a matriz $\tilde{D}$ é obtida pelo produto $\tilde{D}=\alpha I^{s}$, sendo $\alpha$ um escalar com distribuição uniforme no intervalo $(0,1)$ (denota-se $\alpha \sim \mathcal{U}(0,1)$ ).

Para concluir, considere uma matriz $T \in \mathcal{M}^{r}$ não-singular. Então, realiza-se a seguinte transformação de equivalência para que todos os elementos da matriz $A$ sejam reais,

$$
\hat{A}=T \tilde{A} T^{-1}, \quad \hat{B}=T \tilde{B}, \quad \hat{G}=T \tilde{G}, \quad \hat{C}=\left(T^{-1}\right)^{\prime} \tilde{C} T^{-1} \text { e } \hat{D}=\tilde{D}
$$

A matriz de transição de probabilidades, $\mathbb{P}$, é gerada a partir da sua representação na forma canônica apresentada em Çinlar (1975, Equação 3.15).

Por fim, geram-se os vetores $x_{0}$ e $\pi(0)$, sendo que os elementos do vetor $x_{0}$ são escalares com distribuição uniforme no intervalo $(-1,1)$. Para $\pi(0)$, inicialmente são sorteados os escalares $\beta_{i}$ tais que $\beta_{i} \sim \mathcal{U}(0,1), \forall i \in \mathcal{N}$. Os elementos de $\pi(0)$ são dados por $\pi_{i}(0)=\beta_{i} / \sum_{j=1}^{N} \beta_{j}$.

\subsubsection{Intervalo de MS-Estabilizabilidade}

Considere o SLSM $\hat{\boldsymbol{\Phi}}$ (Equação 6.1) com observação completa da variável $\theta(k), k \in$ $\mathcal{K}$. É com base neste sistema que iremos construir os SLSM utilizado nas simulações. 
Para um escalar $\gamma>0$, tem-se que a sequência de matrizes $\gamma \hat{A}=\left\{\gamma \hat{A}_{i}, i \in\right.$ $\mathcal{N}\}$ é tal que $(\gamma \hat{A}, \hat{B}, \mathbb{P})$ pode, em tese, ser MS-estabilizável ou não. Assim, com o fator $\gamma$ é possível determinar um intervalo J que delimita quando o SLSM atende MSestabilizabilidade para $(\gamma \hat{A}, \hat{B}, \mathbb{P})$.

Determinamos as extremidades do intervalo $\mathcal{J}$ da seguinte forma. Primeiramente, considera-se a sequência de matrizes $\mathscr{A}=\bar{\gamma} \hat{A}$ com $\bar{\gamma}$ pequeno o suficiente para garantir que $(\mathscr{A}, \hat{B}, \mathbb{P})$ é MS-estável (e com isso, MS-estabilizável). Em seguida, é gerada uma sequência de fatores $\gamma_{i} \in \mathbb{R}$ tal que

$$
\begin{aligned}
& \gamma_{i}=1,5 \gamma_{i-1} \text { para } i=1, \ldots, n \\
& \gamma_{0}=1
\end{aligned}
$$

sendo que $\left(\gamma_{0} \mathscr{A}, \hat{B}, \mathbb{P}\right)$ é MS-estabilizável e $\left(\gamma_{n} \mathscr{A}, \hat{B}, \mathbb{P}\right)$ não é MS-estabilizável. Logo, o intervalo que delimita a propriedade da MS-estabilizabilidade para $(\gamma \mathscr{A}, \hat{B}, \mathbb{P})$ é dado por

$$
\mathcal{J}=\left[\gamma_{0}, \gamma_{n}\right)
$$

Observação 6.1. A existência do limitante superior do intervalo, $\gamma_{n}$, não é assegurada. De fato, é possível construir exemplos assim. Em parte por isto, imaginávamos que não teríamos este limite superior para a maioria das realizações obtidas para o sistema $\hat{\mathbf{\Phi}}$. Contudo, foi surpreendente que este limite foi encontrado em todos os sistemas gerados.

Observação 6.2. Para verificar se $(\gamma \mathscr{A}, \hat{B}, \mathbb{P})$ é $M S$-estabilizável ou não, pode-se utilizar a equação algébrica de Riccati acoplada, visto que a cadeia de Markov, $\theta(k)$, é observada (COSTA et al., 2005). Logo, se a EARA (Apêndice B) tem solução, para $C, D>0$, então $(\gamma \mathscr{A}, \hat{B}, \mathbb{P})$ é MS-estabilizável.

\subsubsection{Algoritmo para Gerar o SLSM}

Considere o SLSM

$$
\Phi_{\alpha}: \begin{cases}x_{k+1}=A_{\theta(k)} x_{k}+B_{\theta(k)} u_{k}+G_{\theta(k)} w_{k}, & \forall k \geq 0, \\ y_{k}=x_{k}^{\prime} C_{\theta(k)} x_{k}+u_{k}^{\prime} D_{\theta(k)} u_{k}, & \theta(0) \sim \pi(0) .\end{cases}
$$

A partir do SLSM $\hat{\boldsymbol{\Phi}}$ (Equação 6.1) e do intervalo de MS-estabilizabilidade J (Equação 6.4) é possível gerar $\alpha \in \mathbb{Z}_{+}$SLSM $\Phi$ (Equação 2.11) com as propriedades da teoria de controle (controlabilidade, observabilidade e MS-estabilizabilidade). A seguir, é feita uma descrição da construção dos parâmetros de cada SLSM $\boldsymbol{\Phi}_{\alpha}$ para $\alpha=1, \ldots, n_{p}$. 
Considera-se o intervalo $\mathcal{J} \supset \overline{\mathcal{J}}=\left[\gamma_{n-1}, \gamma_{n}\right)$ e aplica-se o método da bisseção com tolerância $\epsilon$ para obter um intervalo $\hat{\mathcal{J}}=\left[e_{i}, e_{f}\right]$ tal que para todo $\gamma \in \hat{\mathcal{J}}$ tem-se que $(\gamma \mathscr{A}, \hat{B}, \mathbb{P})$ é MS-estabilizável.

Para cada SLSM $\Phi_{\alpha}, \alpha=1, \ldots, n_{p}$, a sequência de matrizes $A \in \mathbb{M}^{r}$ é obtida da seguinte forma

$$
A_{i}=\frac{1}{n_{p}}\left(0,9 e_{i} \alpha\right) \mathscr{A}_{i}, \quad \forall i \in \mathcal{N}
$$

sendo que os demais parâmetros dos sistemas $\boldsymbol{\Phi}_{\alpha}$ são iguais ao do SLSM $\hat{\boldsymbol{\Phi}}$ (Equação $6.1)$.

No Algoritmo 6.1 é apresentado o gerador do SLSM $\Phi$ (Equação 6.5). Neste trabalho, considera-se $a=c=2, b=6, d=5, \epsilon=0,01$ e $n_{p}=10$. Além disso, os modos de todos os SLSM gerados são controláveis e observáveis.

Algoritmo 6.1 Gerador de SLSM

Passo 0: Escolha um valor para os escalares $a, b, c, d, \epsilon$ e $n_{p}$.

Passo 1: Gere os parâmetros para o SLSM $\hat{\boldsymbol{\Phi}}$ (Equação 6.1).

Passo 2: Determine o intervalo $\hat{\mathcal{J}}$.

Passo 3: Para cada $\alpha=1, \ldots, n_{p}$ gere a sequência de matrizes $A$ do $\operatorname{SLSM} \boldsymbol{\Phi}_{\alpha}$.

\subsection{Resultados Computacionais}

Nesta seção são apresentadas as avaliações e comparações do desempenho dos métodos de otimização aplicados ao problema do custo de T estágios (Equação 2.26).

Os algoritmos foram implementados em software $M A T L A B^{\circledR}$ na versão 7.6.0.324 e os testes foram realizados em um computador com as seguintes configurações: processador Intel Core 2 Duo com velocidade de $2.4 \mathrm{GHz}$, memória RAM de $3 \mathrm{~GB}$ e sistema operacional Windows ${ }^{\circledR} 7$.

O conjunto de problemas testes foi gerado de acordo com o Gerador de SLSM (Seção 6.1), totalizando 10.000 problemas. O valor do critério de parada $\epsilon$ foi considerado com sendo $10^{-3}$ para os métodos variacional e de direção de busca. Para a estratégia de observação indireta, foi considerado $\epsilon=10^{-3}$ quando $c=\frac{1}{N}$ e para os demais níveis de observação adotou-se $\epsilon=10^{-2}$. Além disso, para os casos em que $\|X(k)\|>10^{150}$ para algum valor de $k \in \mathcal{K}$, e/ou $\left\|\nabla \mathcal{J}_{T}\right\|>10^{150}$ o algoritmo é interrompido. A escolha do horizonte $T$ para cada SLSM foi feita da seguinte forma: seja $\tilde{X}$ o segundo momento 
do sistema associado à sequência de ganhos $\mathbf{K}=\left\{K^{1}, \ldots, K^{N}\right\}$ obtida pela EARA e $\bar{\epsilon}$ um escalar suficientemente pequeno. Para cada $k=0,1, \ldots$ determina-se o valor de $\tilde{X}(k)$ até que para $k=\bar{k}$ a seguinte condição seja satisfeita $\|\tilde{X}(\bar{k})-\tilde{X}(\bar{k}-1)\| \leq \bar{\epsilon}$, então considera-se $T=2 \bar{k}$.

A seguir são descritas as principais características encontradas nos experimentos. Para tal, esta seção foi subdividida em quatro subseções sendo que nas três primeiras subseções é feita uma comparação entre os métodos desenvolvidos e um método da literatura e na quarta é apresentada uma avaliação do desempenho destes métodos.

Na sequência, iremos nos referir aos problemas aos quais cada método foi executado e atendeu o critério de parada dentro de um número máximo de iterações, como problemas "resolvidos" por aquele método. Assim, como o critério de parada não garante que a solução alcançada é ótima, ao dizer que um método resolve um problema não estamos afirmando que o controle obtido tenha custos próximos do valor ótimo, e por isso também analisamos, adiante, a relação entre custos obtidos pelos métodos comparados.

Além disso, para tornar a análise do desempenho dos algoritmos mais interessante, o conjunto de problemas testes foi dividido em duas classes de acordo com o nível de MS-estabilizabilidade: a Classe 1 é composta pelos SLSM considerados "bastante MSestabilizáveis" e a Classe 2 pelos sistemas considerados "pouco MS-estabilizáveis".

Para escolher em qual classe um SLSM pertence, o seguinte procedimento é adotado:

Para cada $\operatorname{SLSM} \Phi_{\alpha}, \alpha=1, \ldots, 10$, gerado tem-se que para $\alpha=1$ o sistema é "bastante MS-estabilizável" e para $\alpha=10$ o sistema é "pouco MS-estabilizável" de acordo a construção dos sistemas (Seção 6.1). Logo, os SLSM $\Phi_{1}$ pertencem a classe 1 e os $\boldsymbol{\Phi}_{10}$ a classe 2. Agora, para atribuir os demais valores de $\alpha$ a uma das classes, utilizamos a quantidade de problemas que o método variacional, o método de direção de busca e a estratégia observação indireta com o método variacional adaptado resolveram, atendendo ao critério de parada $(\mathrm{ACP})$ dentro de um número máximo de iterações. Desse modo, consideramos que os valores de $\alpha$ pertencentes a classe 1 são aqueles em que ambos os métodos resolveram ACP mais de $60 \%$ do total de problemas obtidos para $\alpha=1$, sendo que esse comportamento ocorre para $\alpha \in[1,6]$. Logo, para $\alpha \in[7,10]$ tem-se a Classe 2. Na Figura 6.1 são apresentadas as quantidades de problemas que ambos os métodos resolveram ACP para cada valor de $\alpha$, sendo que os valores de $\alpha$ pertencentes a classe 1 estão apresentados em azul e para os da classe 2 em vermelho. 


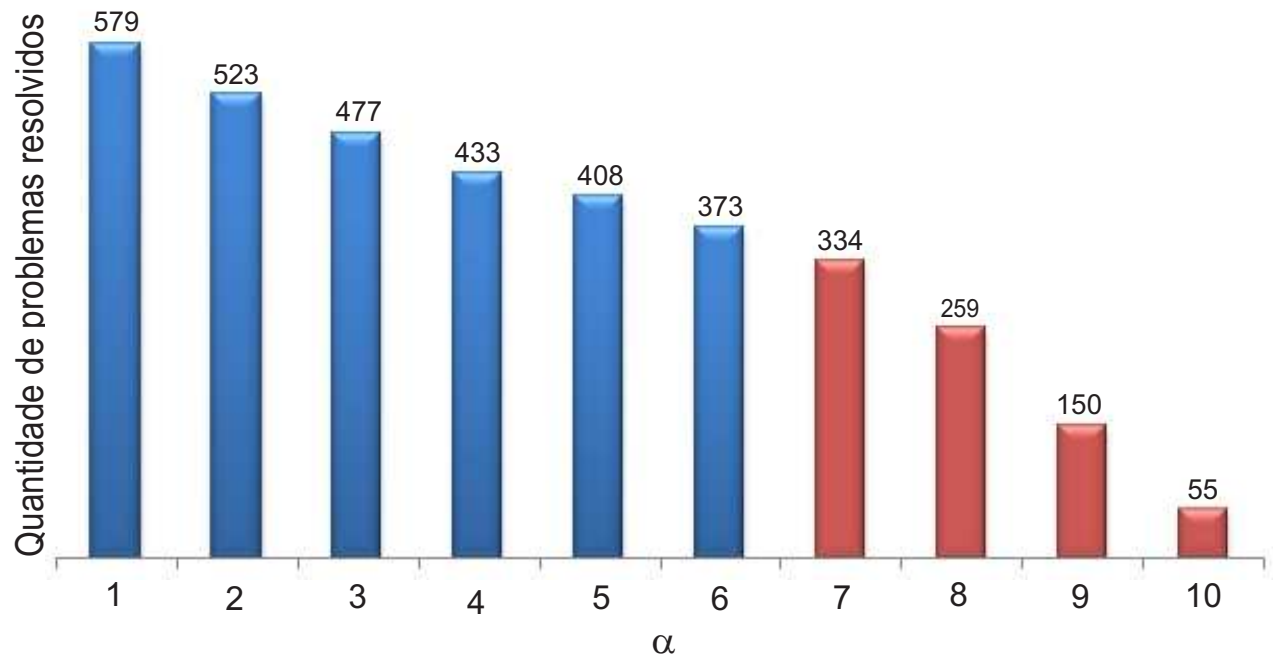

Figura 6.1: Quantidade de problemas que ambos os métodos resolveram ACP versus o valor de $\alpha$

As análises apresentadas nas próximas seções mostram 3 tipos de resultados alcançados pelos métodos, sendo que o primeiro representa os problemas em que os métodos foram executados e atenderam o critério de parada estabelecido dentro de um número máximo de iterações (PACP), o segundo considera os problemas em que os métodos foram executados e realizaram o número máximo de iterações, mas não atingiram o critério de parada estabelecido (PRNMI), e o terceiro abrange os problemas que não foram resolvidos, pois o algoritmo foi interrompido ou não foi possível determinar um ganho inicial (PNR).

\subsubsection{Método Variacional versus Estratégia de Observação Indireta com o Método Variacional Adaptado}

Nesta seção é apresentada uma comparação entre o método variacional, desenvolvido por Vargas et al. (2004) e descrito no Capítulo 3, e a estratégia de observação indireta (Capítulo 5) que utiliza o método variacional adaptado para a observação parcial (Seção 5.2) no passo 1 do Algoritmo 5.1. A avaliação é feita com base nos 10.000 problemas gerados, sendo apresentada de acordo com as classes definidas para o nível de MS-estabilizabilidade.

\section{Classe 1}

Para os SLSM considerados "bastante MS-estabilizáveis", tem-se que o método variacional (MV) resolveu, ACP, 87,80\% dos 6000 problemas, 12,07\% problemas não 
foram resolvidos porque a norma do segundo momento foi maior que a tolerância adotada $\left(\|X(k)\|>10^{150}\right)$ e $0,13 \%$ não alcançaram o critério de parada. A estratégia de observação indireta (EOI), utilizando o MV, resolveu ACP, 79,17\% do total, 20,05\% não foram resolvidos devido a $\|X(k)\|>10^{150}$ e $0,78 \%$ não alcançaram o critério de parada. A Figura 6.2 ilustra estes dados graficamente.

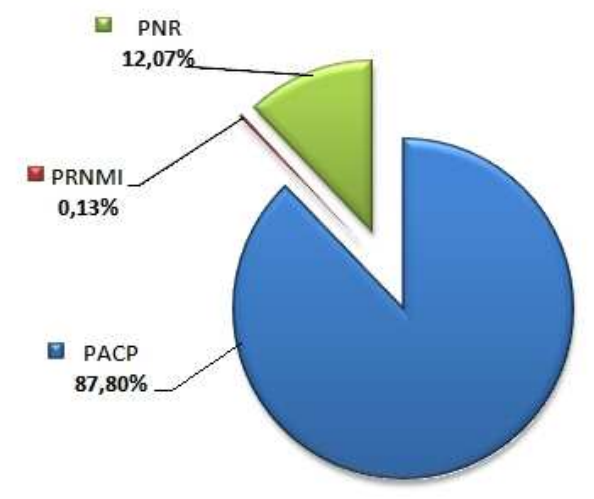

(a) Método Variacional

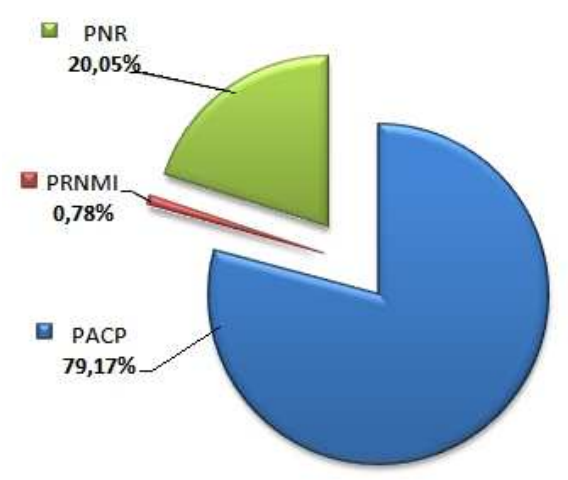

(b) Observação Indireta (MV)

Figura 6.2: Resultados obtidos por cada método, na Classe 1

Analisando somente os problemas que o MV resolveu ACP, tem-se que 89,18\% também foram resolvidos ACP pela EOI (MV), 0,7\% não atenderam o critério de parada e 10,12\% tiveram a norma de $X(k)$ maior que a tolerância adotada. Para os problemas que a EOI (MV) resolveu ACP, tem-se que 98,91\% também foram resolvidos ACP pelo $\mathrm{MV}$, sendo que o restante, $1,09 \%$, não foram resolvidos devido a $\|X(k)\|>10^{150}$. Na Figura 6.3 são ilustrados estes dados.

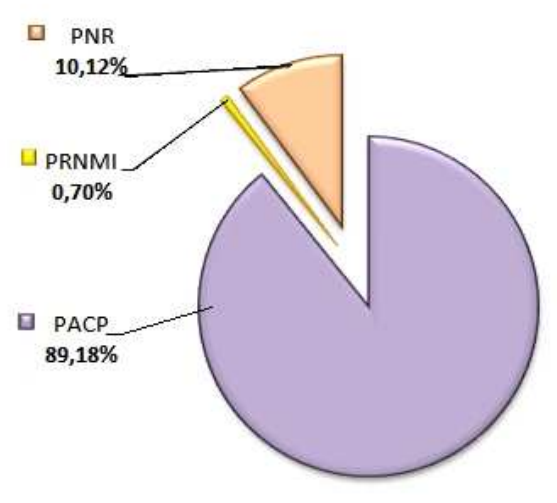

(a) Método Variacional

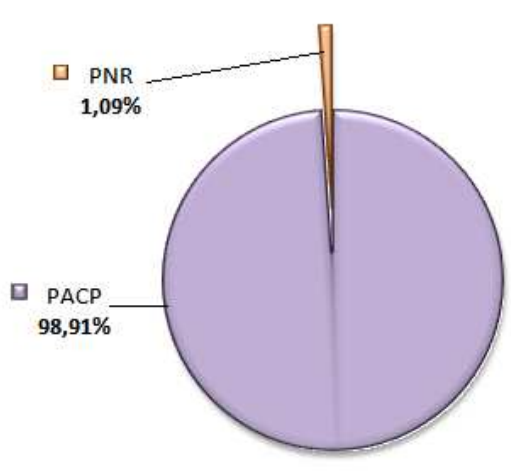

(b) Observação Indireta (MV)

Figura 6.3: Comparação dos resultados de cada método, para a Classe 1 
Na Figura 6.4 está apresentado o comportamento do custo de T estágios obtido pelo MV, $\mathcal{J}_{M V}$, em relação ao obtido pela EOI (MV), $\mathcal{J}_{E O I}$. Note que a partir do gráfico não é possível afirmar qual dos métodos teve o melhor desempenho em relação aos resultados obtidos. Assim, será utilizado o teste de hipótese (MAGALHÃES, LIMA, 2002) para formular uma suposição a respeito da média da razão entre os custos, a qual será testada com base nos dados obtidos pelo métodos.

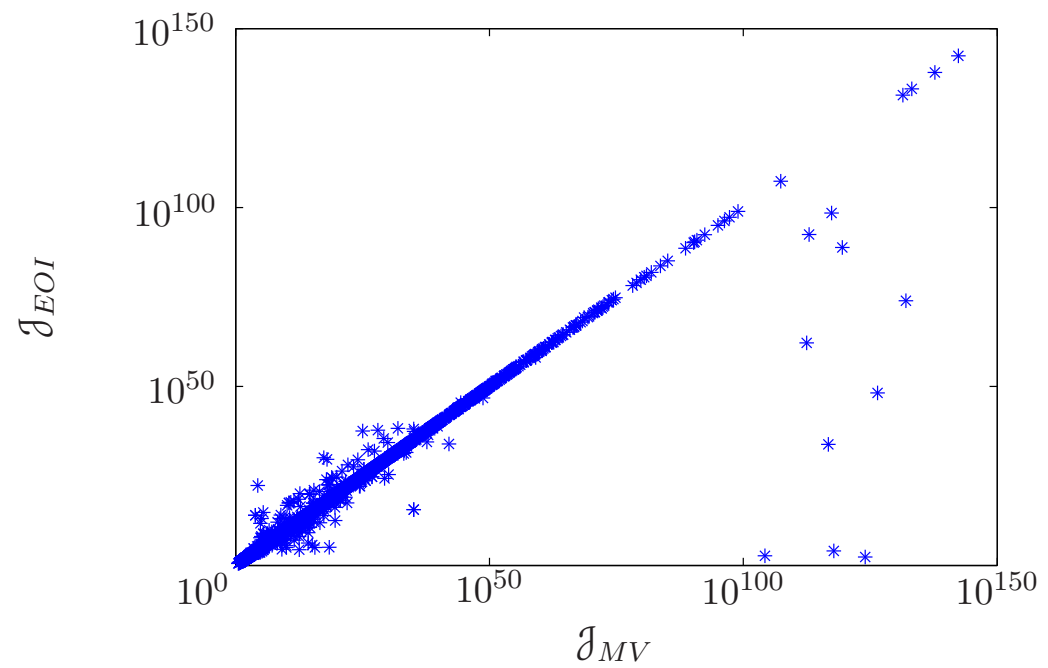

Figura 6.4: Custo do MV versus o da EOI (MV), para a Classe 1

Teste de Hipótese 6.1. Deseja-se testar se a média $\mu$, razão entre custo em escala logarítmica do $M V$ e da EOI (MV), é igual a 1, contra a alternativa de ser maior que 1. Inferimos através das amostras (custos obtidos) que o desvio padrão do custo é dado por $\sigma \approx 1,0307$. As duas hipóteses sobre a média da amostra são denotadas por $H_{0}$ (Hipótese Nula) e $H_{a}$ (Hipótese Alternativa), respectivamente. Assim,

$$
\begin{array}{ll}
H_{0}: \mu=1 & \left(\mathcal{J}_{M V} \leq \mathcal{J}_{E O I}\right) \\
H_{a}: \mu>1 & \left(\mathcal{J}_{M V}>\mathcal{J}_{E O I}\right) .
\end{array}
$$

$O$ erro ao rejeitar a hipótese $H_{0}$ quando, na realidade, $H_{0}$ é verdadeira é dado por

$$
\alpha=P\left[\text { rejeitar } H_{0} \mid H_{0} \text { verdadeira }\right]=P\left[\bar{X}>x_{c} \mid H_{0}\right]=P\left[Z>\frac{x_{c}-\mu}{S}\right],
$$

sendo que $Z=\frac{\bar{X}-\mu}{S}$ com $Z \sim N(0,1)$ e $S=\frac{\sigma}{\sqrt{4698}}$ (estamos aproximando a distribuição de $Z$ por uma normal pois o número de amostras é grande). Logo, considerando o valor crítico $x_{c}$ igual a 1,02 , temos

$$
\alpha=P\left[Z>\frac{0,02}{0,0150}\right] \approx P[Z>1,3333] \approx 9,12 \% .
$$


O valor obtido para a média foi $\mu=1,0290$, de forma que $\mu>x_{c}$ implica em rejeitar a Hipótese Nula e concluir que a EOI (MV) obteve custos menores. A probabilidade de estarmos enganados nesta conclusão é de $\alpha=9,12 \%$.

Na Figura 6.5 está apresentado o comportamento do tempo de CPU obtido pelo $\mathrm{MV}, T_{M V}$, em relação ao obtido pela EOI (MV), $T_{E O I}$. Além disso, no Teste de Hipótese 6.2 é formulada uma suposição a respeito da média da razão entre os tempos obtidos pelo métodos.

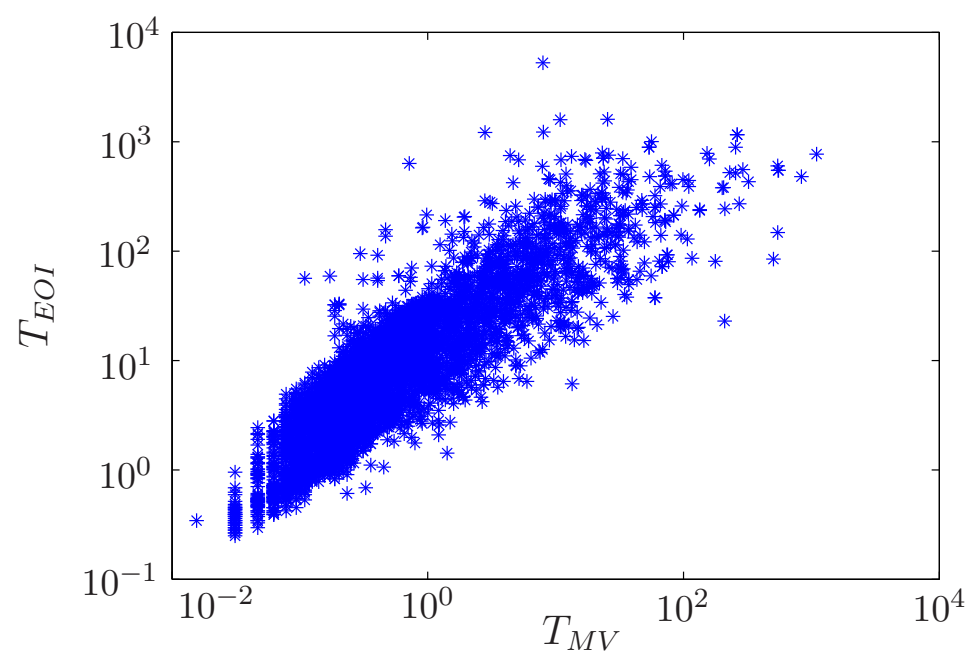

Figura 6.5: Tempo de CPU do MV versus o da EOI (MV), para a Classe 1

Teste de Hipótese 6.2. Deseja-se testar se a média $\mu$, razão entre tempo de CPU do $M V$ e o tempo da EOI (MV), é igual a 0,1, contra a alternativa de ser maior que 0,1 . Inferimos através das amostras (tempos obtidos) que o desvio padrão do tempo é dado por $\sigma \approx 0,2372$. As duas hipóteses sobre a média da amostra são denotadas por $H_{0}$ e $H_{a}$, respectivamente. Assim,

$$
\begin{aligned}
& H_{0}: \mu=0,1\left(T_{E O I}=10 T_{M V}\right) \\
& H_{a}: \mu>0,1\left(T_{E O I}>10 T_{M V}\right) .
\end{aligned}
$$

$O$ erro ao rejeitar a hipótese $H_{0}$ quando, na realidade, $H_{0}$ é verdadeira é dado por

$$
\alpha=P\left[\text { rejeitar } H_{0} \mid H_{0} \text { verdadeira }\right] \approx P[Z>2,8571] \approx 0,21 \%,
$$

sendo que $Z=\frac{\bar{X}-\mu}{S}$ com $Z \sim(0,1)$ e $S=\frac{\sigma}{\sqrt{4698}}$ (estamos aproximando a distribuição de $Z$ por uma normal pois o número de amostras é grande) e considerando o valor crítico $x_{c}$ igual a 0,11. O valor obtido para a média foi $\mu=0,1062$, de forma que $\mu<x_{c}$ 
implica em aceitar a Hipótese Nula e concluir que a o tempo de CPU da EOI (MV) é igual a 10 vezes o tempo de CPU do MV. A probabilidade de estarmos enganados nesta conclusão é de $\alpha=0,21 \%$.

\section{Classe 2}

Para os sistemas "pouco MS-estabilizáveis", tem-se que o método variacional resolveu, ACP, 62,30\% dos 4000 problemas, 0,20\% problemas não alcançaram o critério de parada e 37,50\% não foram resolvidos porque a norma do segundo momento foi maior que a tolerância adotada. A estratégia de observação indireta, utilizando o método variacional, resolveu ACP 47,28\% do total, 50,60\% não foram resolvidos devido a $\|X(k)\|>10^{150}$ e $2,12 \%$ não alcançaram o critério de parada. A Figura 6.6 ilustra estes dados graficamente.

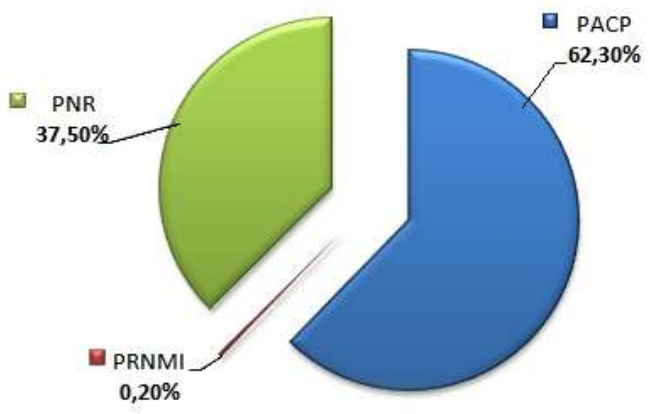

(a) Método Variacional

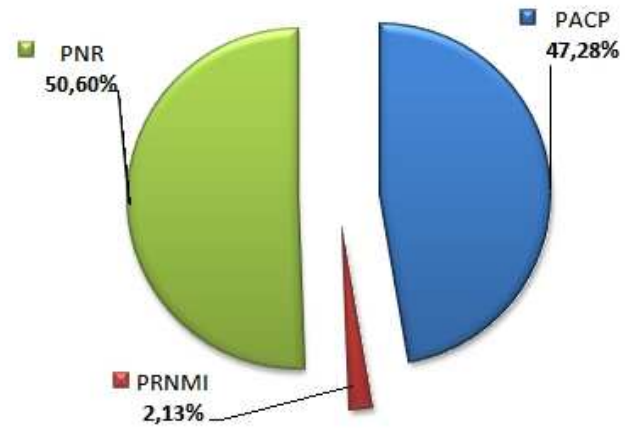

(b) Observação Indireta (MV)

Figura 6.6: Resultados obtidos por cada método, para a Classe 2

Analisando somente os problemas que o MV resolveu ACP, tem-se que 71,87\% também foram resolvidos, ACP, pela EOI (MV), 3,01\% não atenderam o critério de parada e $25,12 \%$ tiveram a norma de $X(k)$ maior que a tolerância adotada. Para os problemas que a EOI (MV) resolveu ACP, tem-se que 94,71\% também foram resolvidos pelo MV, sendo que o restante, $5,29 \%$, não foram resolvidos devido a $\|X(k)\|>10^{150}$. $\mathrm{Na}$ Figura 6.7 são ilustrados estes dados. 


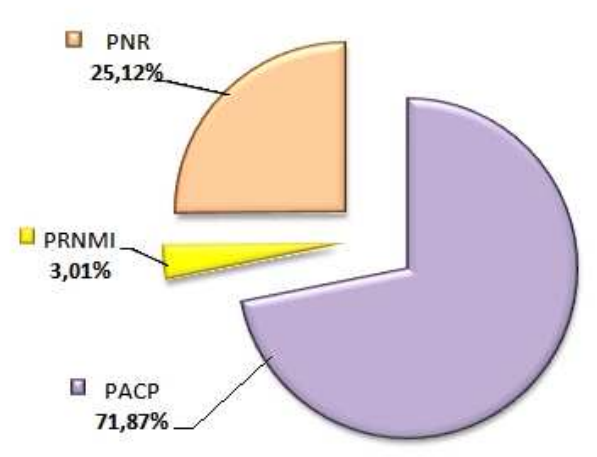

(a) Método Variacional

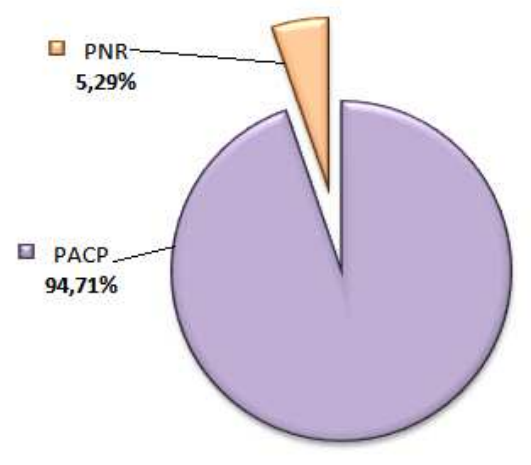

(b) Observação Indireta (MV)

Figura 6.7: Comparação dos resultados de cada método, para a Classe 2

Na Figura 6.8 está apresentado o comportamento do custo de T estágios obtido pelo $M V, \mathcal{J}_{M V}$, em relação ao obtido pela EOI (MV), $\mathcal{J}_{E O I}$, sendo que uma suposição é formulada a respeito da média da razão entre os custos obtidos pelo métodos no Teste de Hipótese 6.3.

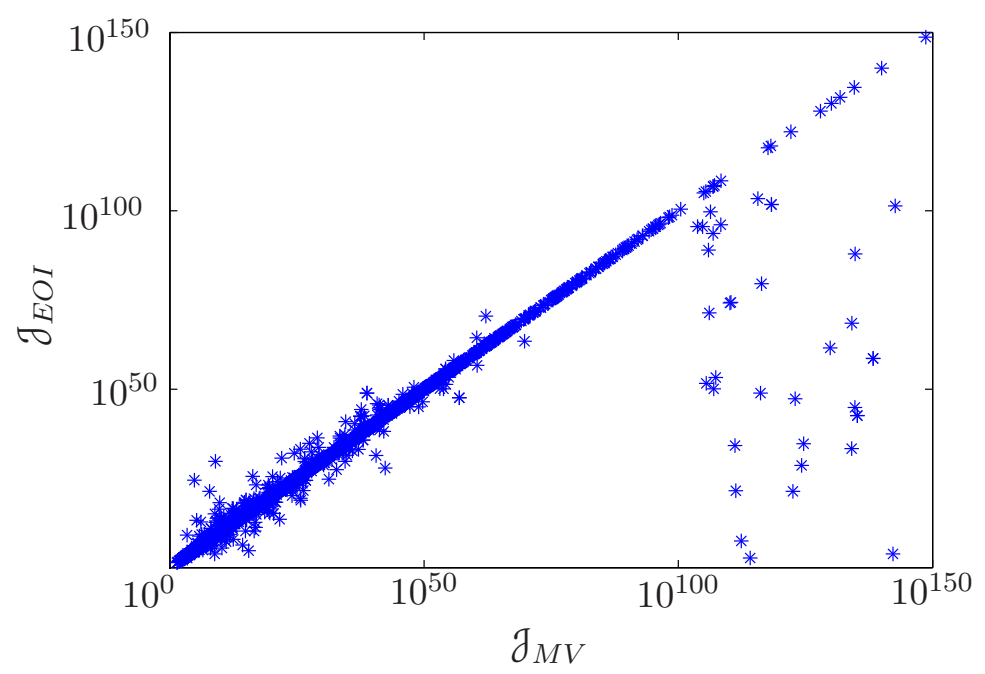

Figura 6.8: Custo do MV versus o da EOI (MV), para a Classe 2

Teste de Hipótese 6.3. Deseja-se testar se a média $\mu$, razão entre custo em escala logarítmica do $M V$ e da EOI (MV), é igual a 1, contra a alternativa de ser maior que 1. Inferimos através das amostras (custos obtidos) que o desvio padrão é dado por $\sigma \approx 1,3618$. As duas hipóteses sobre a média da amostra são denotadas por $H_{0}$ e $H_{a}$, 
respectivamente. Assim,

$$
\begin{aligned}
& H_{0}: \mu=1 \quad\left(\mathcal{J}_{M V} \leq \mathcal{J}_{E O I}\right) \\
& H_{a}: \mu>1 \quad\left(\mathcal{J}_{M V}>\mathcal{J}_{E O I}\right) .
\end{aligned}
$$

$O$ erro ao rejeitar a hipótese $H_{0}$ quando, na realidade, $H_{0}$ é verdadeira é dado por

$$
\alpha=P\left[\text { rejeitar } H_{0} \mid H_{0} \text { verdadeira }\right] \approx P[Z>2,1739] \approx 1,48 \%,
$$

sendo que $Z=\frac{\bar{X}-\mu}{S}$ com $Z \sim(0,1)$ e $S=\frac{\sigma}{\sqrt{1791}}$ (estamos aproximando a distribuição de $Z$ por uma normal pois o número de amostras é grande) e considerando o valor crítico $x_{c}=1,07$. O valor obtido para a média foi $\mu=1,0750$, de forma que $\mu>x_{c}$ implica em rejeitar a Hipótese Nula e concluir que a EOI (MV) obteve custos menores. A probabilidade de estarmos enganados nesta conclusão é de $\alpha=1,48 \%$. Este valor é bem menor do que para a Classe 1, sugerindo que o EOI (MV) alcança menores custos em relação ao $M V$ em cenários mais desfavoráveis (sistemas com menor grau de MS-estabilizabilidade).

Na Figura 6.9 está apresentado o comportamento do tempo de CPU obtido pelo $\mathrm{MV}, T_{M V}$, em relação ao obtido pela EOI (MV), $T_{E O I}$. Além disso, no Teste de Hipótese 6.4 é formulada uma suposição a respeito da média da razão entre os tempos obtidos pelo métodos.

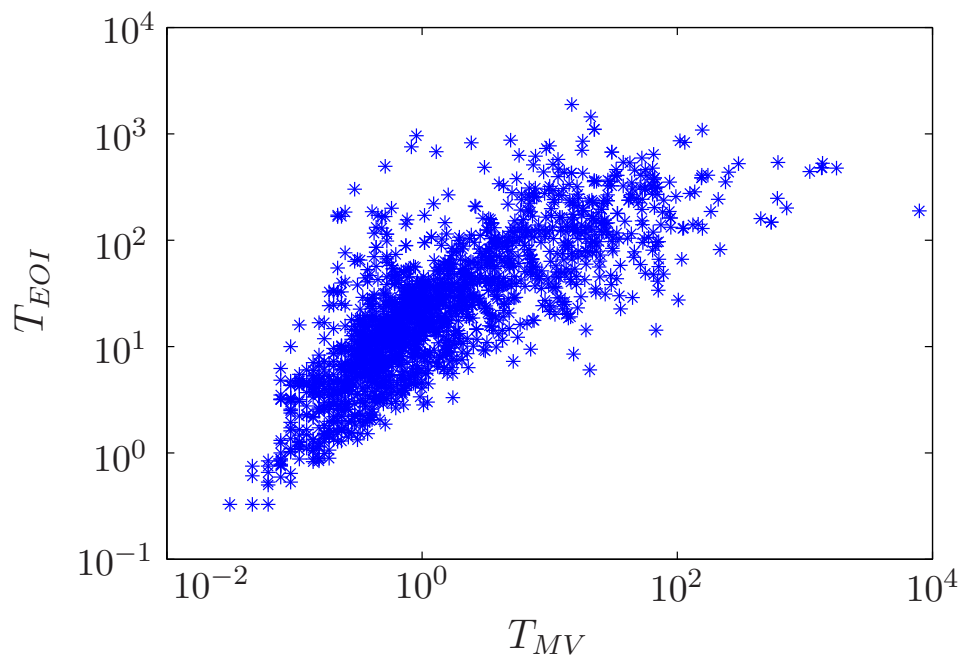

Figura 6.9: Tempo de CPU do MV versus o da EOI (MV), para a Classe 2

Teste de Hipótese 6.4. Deseja-se testar se a média $\mu$, razão entre tempo de CPU do $M V$ e o tempo da EOI (MV), é igual a 0,1, contra a alternativa de ser maior que 
0,1. Inferimos através das amostras (tempos obtidos) que o desvio padrão é dado por $\sigma \approx 1,0479$. As duas hipóteses sobre a média da amostra são denotadas por $H_{0}$ e $H_{a}$, respectivamente. Assim,

$$
\begin{aligned}
& H_{0}: \mu=0,1\left(T_{E O I}=10 T_{M V}\right) \\
& H_{a}: \mu>0,1\left(T_{E O I}>10 T_{M V}\right) .
\end{aligned}
$$

$O$ erro ao rejeitar a hipótese $H_{0}$ quando, na realidade, $H_{0}$ é verdadeira é dado por

$$
\alpha=P\left[\text { rejeitar } H_{0} \mid H_{0} \text { verdadeira }\right] \approx P[Z>0,202] \approx 2,18 \%,
$$

sendo que $Z=\frac{\bar{X}-\mu}{S} \operatorname{com} Z \sim N(0,1)$ e $S=\frac{\sigma}{\sqrt{1791}}$ (estamos aproximando a distribuição de $Z$ por uma normal pois o número de amostras é grande) e considerando o valor crítico $x_{c}$ igual a 0,15. O valor obtido para a média foi $\mu=0,1648$, de forma que $\mu>x_{c}$ implica em rejeitar a Hipótese Nula e concluir que o tempo de CPU da EOI $(M V)$ é maior que 10 vezes o tempo de CPU do MV. A probabilidade de estarmos enganados nesta conclusão é de $\alpha=2,18 \%$.

\subsubsection{Método Variacional versus Método de Direção de Busca}

Nesta seção é apresentada uma comparação entre o método variacional (Capítulo 3) e o método de direção de busca (Capítulo 4). A avaliação é feita com base nos 10.000 problemas gerados, sendo apresentada de acordo com as classes definidas para o nível de MS-estabilizabilidade.

\section{Classe 1}

Para esta classe tem-se que o método de Newton (MN) resolveu, ACP, 48,24\% dos 6000 problemas e 19,18\% não alcançaram o critério de parada. Os problemas não resolvidos pelo MN representam um total de 32,58\%, sendo que 13,85\% não foram resolvidos devido a $\|X(k)\|>10^{150}(3,72 \%)$ ou $\left\|\nabla \mathcal{J}_{T}\right\|>10^{150}(10,13 \%)$ e para $18,73 \%$ não foi possível determinar um ganho para inicializar o método (a norma do gradiente dos possíveis candidatos é maior $10^{150}$ ). Os dados referentes ao método variacional foram apresentados na Seção 6.2.1 e serão omitidos. A Figura 6.10 ilustra estes dados graficamente. 


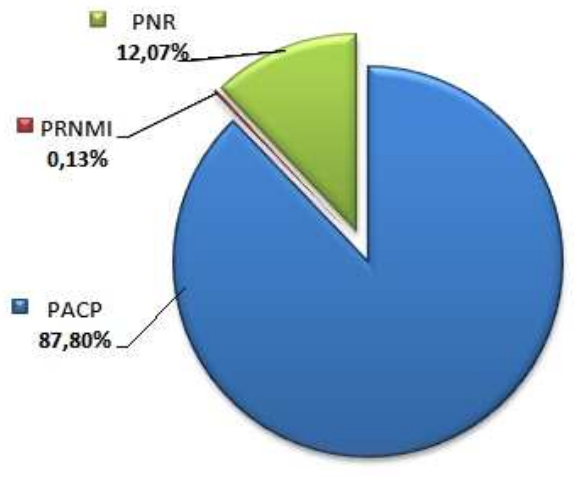

(a) Método Variacional

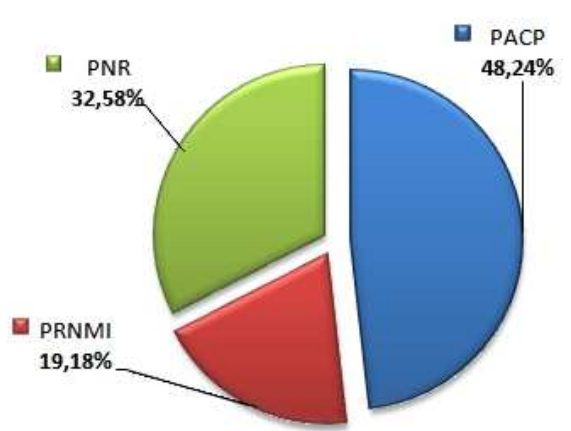

(b) Método de Newton

Figura 6.10: Resultados obtidos por cada método, para a Classe 1

Analisando somente os problemas que o MV resolveu ACP, tem-se que 54,16\% também foram resolvidos ACP pelo MN e 21,30\% não atenderam o critério de parada. Os problemas não resolvidos pela MN representam um total 24,54\%, sendo que 14,31\% não foram resolvidos devido $\|X(k)\|>10^{150}(3,51 \%)$ ou $\left\|\nabla \mathcal{J}_{T}\right\|>10^{150}(10,80 \%) \mathrm{e}$ para 10,23\% não foi possível determinar um ganho inicial. Para os problemas que o MN resolveu ACP, tem-se que 98,58\% também foram resolvidos ACP pelo MV, sendo que o restante, $1,42 \%$, não foram resolvidos devido a $\|X(k)\|>10^{150}$. Na Figura 6.11 são ilustrados estes dados.

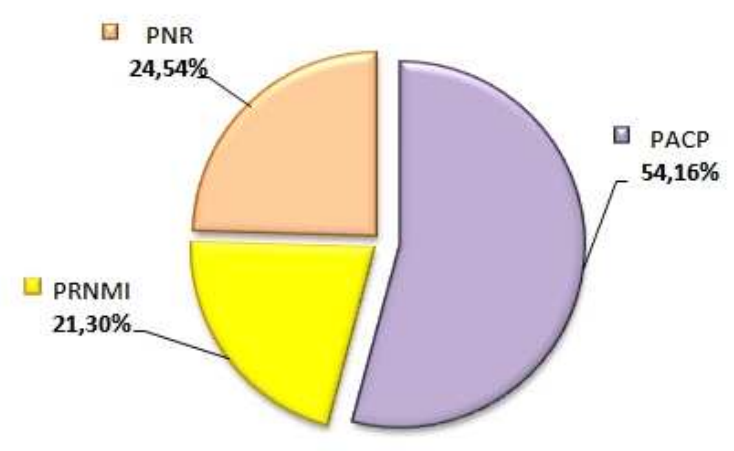

(a) Método Variacional

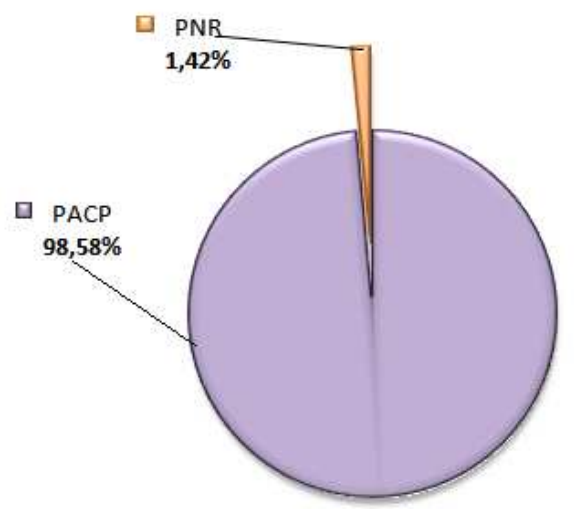

(b) Método de Newton

Figura 6.11: Comparação dos resultados de cada método, para a Classe 1 
Na Figura 6.12 está apresentado o comportamento do custo de T estágios obtido pelo método variacional, $\mathcal{J}_{M V}$, em relação ao obtido pelo método de Newton, $\mathcal{J}_{M N}$, sendo que no Teste de Hipótese 6.5 é apresentada uma suposição formulada a respeito da média da razão entre os custos obtidos pelo métodos.

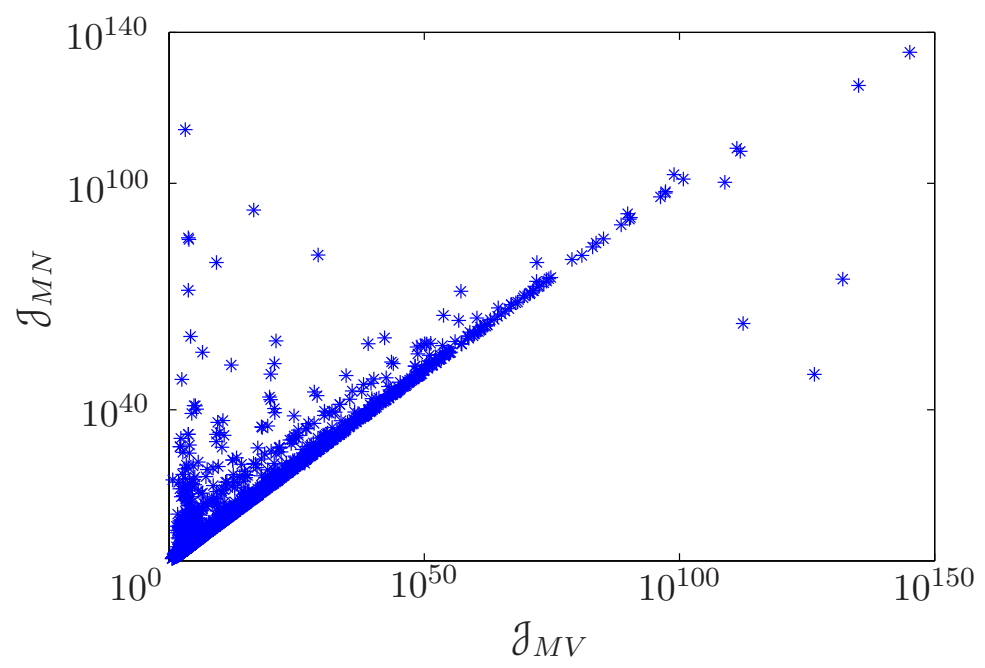

Figura 6.12: Custo do MV versus o do MN, para a Classe 1

Teste de Hipótese 6.5. Deseja-se testar se a média $\mu$, razão entre custo em escala logarítmica do $M V$ e do $M N$, é igual a 1, contra a alternativa de ser maior que 1. Inferimos através das amostras (custos obtidos) que o desvio padrão do custo é dado por $\sigma \approx 1,1875$. As duas hipóteses sobre a média da amostra são denotadas por $H_{0}$ (Hipótese Nula) e $H_{a}$ (Hipótese Alternativa), respectivamente. Assim,

$$
\begin{aligned}
& H_{0}: \mu=1 \quad\left(\mathcal{J}_{M V} \leq \mathcal{J}_{M N}\right) \\
& H_{a}: \mu>1 \quad\left(\mathcal{J}_{M V}>\mathcal{J}_{M N}\right) .
\end{aligned}
$$

$O$ erro ao rejeitar a hipótese $H_{0}$ quando, na realidade, $H_{0}$ é verdadeira é dado por

$$
\alpha=P\left[\text { rejeitar } H_{0} \mid H_{0} \text { verdadeira }\right] \approx P[Z>2,8571] \approx 0,21 \%,
$$

sendo que $Z=\frac{\bar{X}-\mu}{S}$ com $Z \sim N(0,1), S=\frac{\sigma}{\sqrt{2853}}$ (estamos aproximando a distribuição de $Z$ por uma normal pois o número de amostras é grande) e considerando o valor crítico $x_{c}$ igual a 1,02. O valor obtido para a média foi $\mu=0,9135$, de forma que $\mu<x_{c}$ implica em aceitar a Hipótese Nula e concluir que os métodos são equivalentes, pois os custos obtidos pelo MN são maiores ou iguais aos do MV. A probabilidade de estarmos enganados nesta conclusão é de $\alpha=0,21 \%$. 
Na Figura 6.13 está apresentado o comportamento do tempo de CPU obtido pelo MV, $T_{M V}$, em relação ao obtido pelo $\mathrm{MN}, T_{M N}$. Além disso, no Teste de Hipótese 6.6 é formulada uma suposição a respeito da média da razão entre os tempos obtidos pelo métodos.

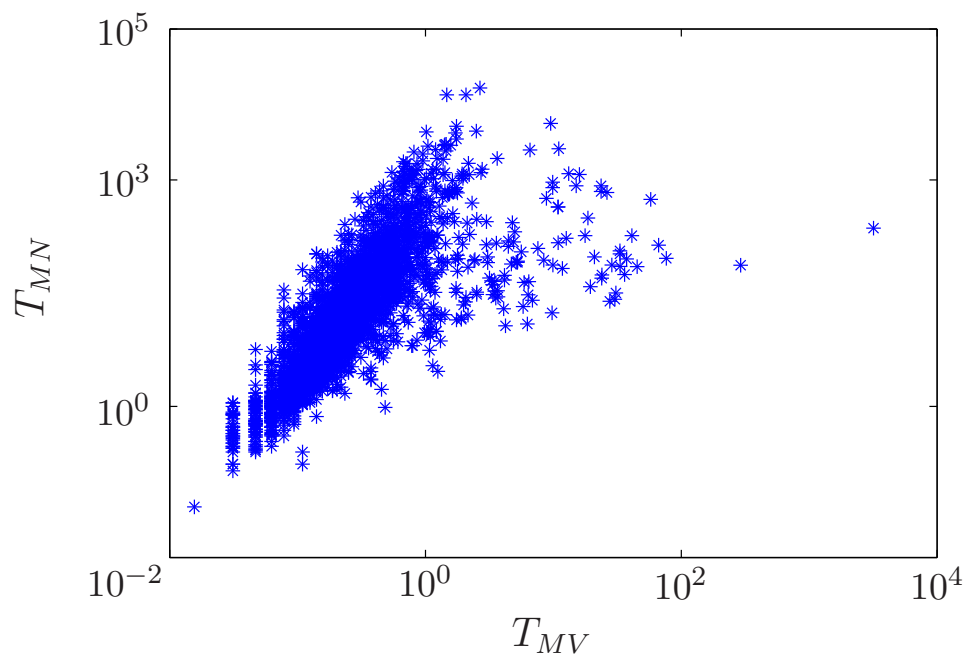

Figura 6.13: Tempo de $C P U$ do $M V$ versus o do $M N$, para a Classe 1

Teste de Hipótese 6.6. Deseja-se testar se a média $\mu$, razão entre tempo de $C P U$ do $M V$ e o tempo da MN, é igual a 0,04, contra a alternativa de ser maior que 0,04. Inferimos através das amostras (tempos obtidos) que o desvio padrão do tempo é dado por $\sigma \approx 0,2748$. As duas hipóteses sobre a média da amostra são denotadas por $H_{0} e$ $H_{a}$, respectivamente. Assim,

$$
\begin{aligned}
& H_{0}: \mu=0,04\left(T_{M N}=25 T_{M V}\right) \\
& H_{a}: \mu>0,04\left(T_{M N}>25 T_{M V}\right) .
\end{aligned}
$$

$O$ erro ao rejeitar a hipótese $H_{0}$ quando, na realidade, $H_{0}$ é verdadeira é dado por

$$
\alpha=P\left[\text { rejeitar } H_{0} \mid H_{0} \text { verdadeira }\right] \approx P[Z>3,09216] \approx 0,004 \%,
$$

sendo que $Z=\frac{\bar{X}-\mu}{S}$ com $Z \sim N(0,1), S=\frac{\sigma}{\sqrt{2853}}$ (estamos aproximando a distribuição de $Z$ por uma normal pois o número de amostras é grande) e considerando o valor crítico $x_{c}$ igual a 0,06. O valor obtido para a média foi $\mu=0,0405$, de forma que $\mu<x_{c}$ implica em aceitar a Hipótese Nula e concluir que o tempo de CPU do MNé igual a 25 vezes o tempo de CPU do MV. A probabilidade de estarmos enganados nesta conclusão é de $\alpha=0,004 \%$. 


\section{Classe 2}

Para esta classe tem-se que o método de Newton resolveu, ACP, 24,22\% dos 4000 problemas e 15,28\% não alcançaram o critério de parada. Os problemas não resolvidos pelo MN representam um total de 60,50\%, sendo que 14,80\% não foram resolvidos devido a $\|X(k)\|>10^{150}(4,93 \%)$ ou $\left\|\nabla \mathcal{J}_{T}\right\|>10^{150}(9,87 \%)$ e para $45,70 \%$ não foi possível determinar um ganho inicial. Os dados referentes ao método variacional foram apresentados na Seção 6.2.1 e serão omitidos. A Figura 6.14 ilustra estes dados graficamente.

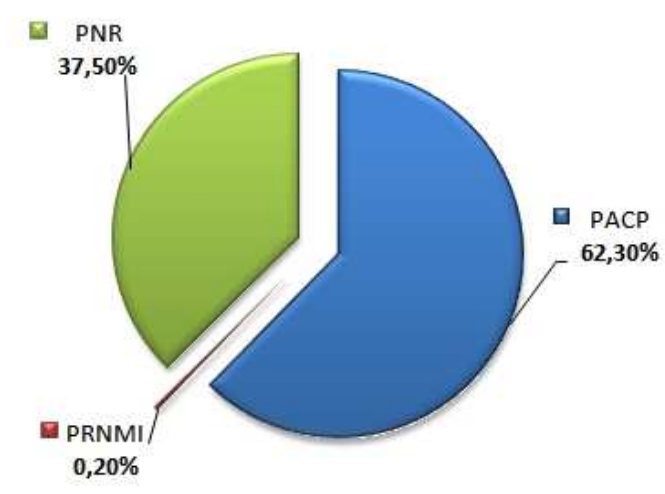

(a) Método Variacional

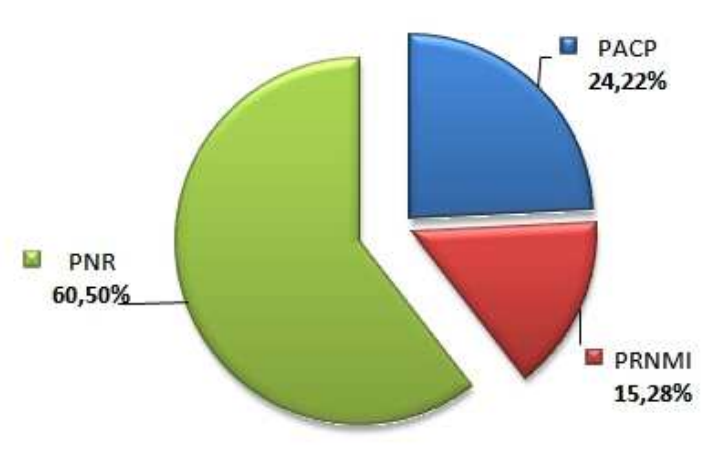

(b) Método de Newton

Figura 6.14: Resultados obtidos por cada método, para a Classe 2

Analisando somente os problemas que o MV resolveu ACP, tem-se que 36,00\% também foram resolvidos ACP pelo MN e 22,35\% não atenderam o critério de parada. Os problema não resolvidos pelo MN representam um total de 41,65\%, sendo que $18,10 \%$ não foram resolvidos devido a $\|X(k)\|>10^{150}(5,01 \%)$ ou $\left\|\nabla \mathcal{J}_{T}\right\|>10^{150}$ $(13,00 \%)$ e para $23,55 \%$ não foi possível determinar um ganho inicial. Para os problemas que o MN resolveu ACP, tem-se que 92,57\% também foram resolvidos ACP pelo MV, 0,21\% não atenderam o critério de parada e 7,22\%, não foram resolvidos devido a $\|X(k)\|>10^{150}$. Na Figura 6.15 são ilustrados estes dados. 


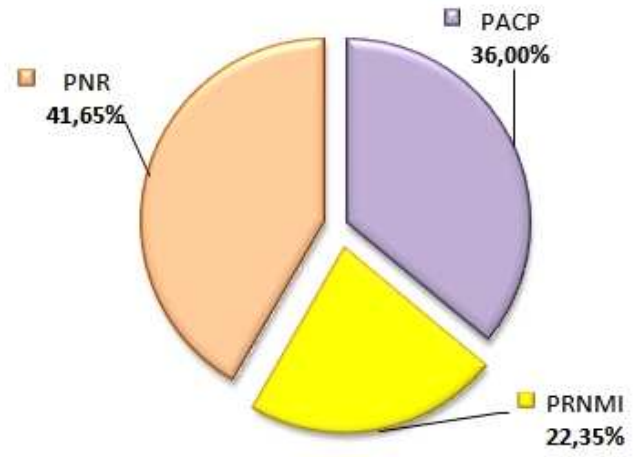

(a) Método Variacional

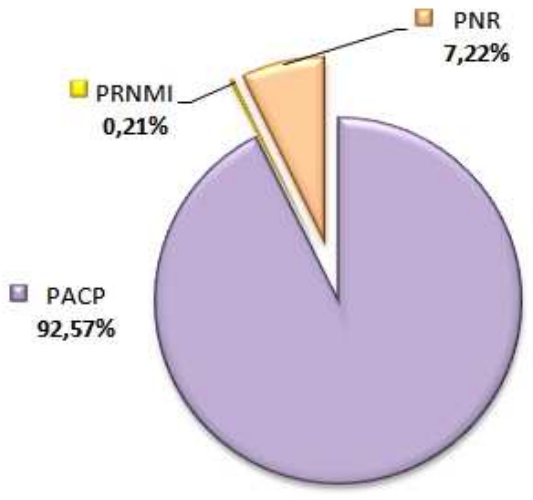

(b) Método de Newton

Figura 6.15: Comparação dos resultados de cada método, para a Classe 2

Na Figura 6.16 está apresentado o comportamento do custo de T estágios obtido pelo método variacional, $\mathcal{J}_{M V}$, em relação ao obtido pelo método de Newton, $\mathcal{J}_{M N}$, sendo que no Teste de Hipótese 6.7 é apresentada uma suposição formulada a respeito da média da razão entre os custos obtidos pelo métodos.

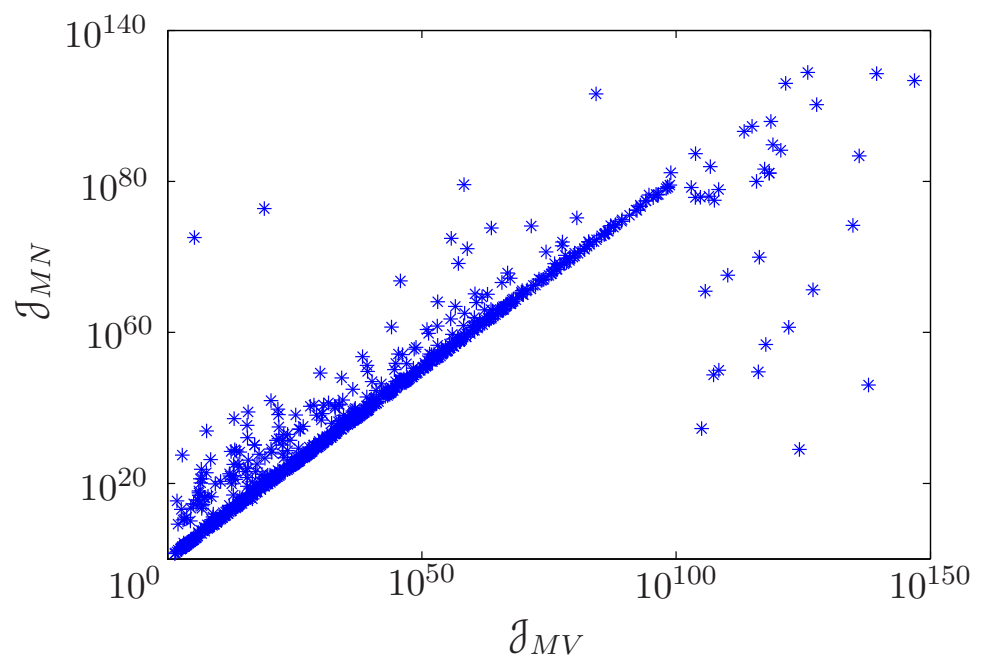

Figura 6.16: Custo do MV versus o do MN, para a Classe 2

Teste de Hipótese 6.7. Deseja-se testar se a média $\mu$, razão entre custo em escala logarítmica do $M V$ e do $M N$, é igual a 1, contra a alternativa de ser maior que 1. Inferimos através das amostras (custos obtidos) que o desvio padrão é dado por $\sigma \approx$ 0,2380. As duas hipóteses sobre a média da amostra são denotadas por $H_{0}$ e $H_{a}$, 
respectivamente. Assim,

$$
\begin{aligned}
& H_{0}: \mu=1 \quad\left(\mathcal{J}_{M V} \leq \mathcal{J}_{M N}\right) \\
& H_{a}: \mu>1 \quad\left(\mathcal{J}_{M V}>\mathcal{J}_{M N}\right) .
\end{aligned}
$$

$O$ erro ao rejeitar a hipótese $H_{0}$ quando, na realidade, $H_{0}$ é verdadeira é dado por

$$
\alpha=P\left[\text { rejeitar } H_{0} \mid H_{0} \text { verdadeira }\right] \approx P[Z>2,5316] \approx 0,56 \%,
$$

sendo que $Z=\frac{\bar{X}-\mu}{S}$ com $Z \sim(0,1), S=\frac{\sigma}{\sqrt{897}}$ (estamos aproximando a distribuição de $Z$ por uma normal pois o número de amostras é grande) e considerando o valor crítico $x_{c}$ igual a 1,02. O valor obtido para a média foi $\mu=0,9445$, de forma que $\mu<x_{c}$ implica em aceitar a Hipótese Nula e concluir que os métodos são equivalentes. A probabilidade de estarmos enganados nesta conclusão é de $\alpha=0,56 \%$.

Na Figura 6.17 está apresentado o comportamento do tempo de CPU obtido pelo MV, $T_{M V}$, em relação ao obtido pelo MN, $T_{M N}$. Além disso, no Teste de Hipótese 6.8 é formulada uma suposição a respeito da média da razão entre os tempos obtidos pelo métodos.

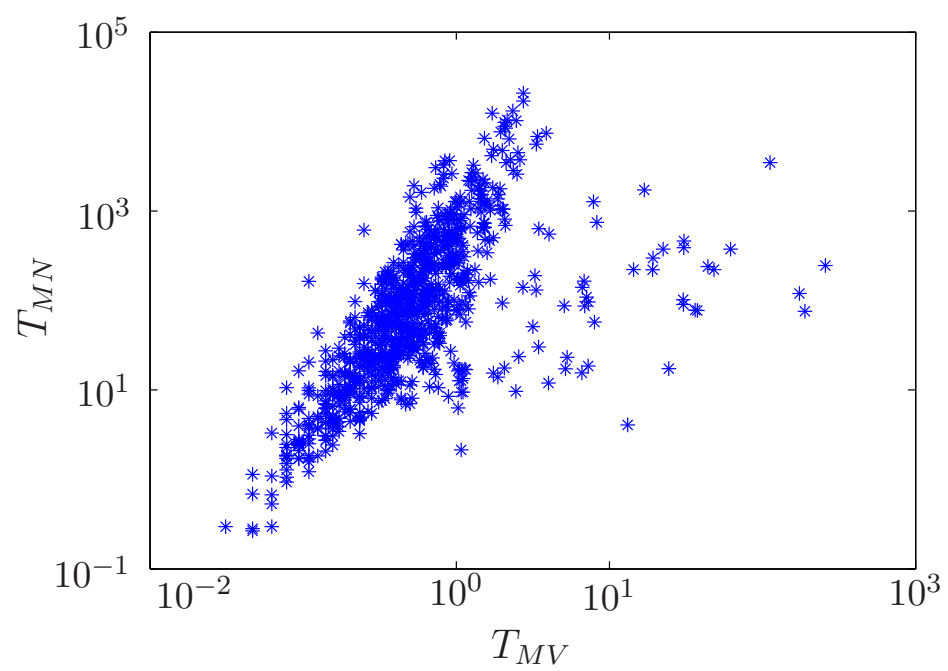

Figura 6.17: Tempo de $C P U$ do $M V$ versus o do $M N$, para a Classe 2

Teste de Hipótese 6.8. Deseja-se testar se a média $\mu$, razão entre tempo de $C P U$ do $M V$ e o tempo da MN, é igual a 0,03, contra a alternativa de ser maior que 0,03. Inferimos através das amostras (custos obtidos) que o desvio padrão é dado por $\sigma \approx$ 0,1620. As duas hipóteses sobre a média da amostra são denotadas por $H_{0}$ e $H_{a}$, 
respectivamente. Assim,

$$
\begin{aligned}
& H_{0}: \mu=0,03\left(T_{M N}=33 T_{M V}\right) \\
& H_{a}: \mu>0,03\left(T_{M N}>33 T_{M V}\right) .
\end{aligned}
$$

O erro ao rejeitar a hipótese $H_{0}$ quando, na realidade, $H_{0}$ é verdadeira é dado por

$$
\alpha=P\left[\text { rejeitar } H_{0} \mid H_{0} \text { verdadeira }\right] \approx P[Z>3,7037] \approx 0,01 \% \text {, }
$$

sendo que $Z=\frac{\bar{X}-\mu}{S}$ com $Z \sim N(0,1), S=\frac{\sigma}{\sqrt{897}}$ (estamos aproximando a distribuição de $Z$ por uma normal pois o número de amostras é grande) e considerando o valor crítico $x_{c}$ igual a 0,05. O valor obtido para a média foi $\mu=0,0318$, de forma que $\mu>x_{c}$ implica em rejeitar a Hipótese Nula e concluir que o tempo de CPU do MNé maior que 33 vezes o tempo de CPU do MV. A probabilidade de estarmos enganados nesta conclusão é de $\alpha=0,01 \%$.

\subsubsection{Método Variacional versus Estratégia de Observação Indireta com Método de Direção de Busca Adaptado}

Nesta seção é apresentada uma comparação entre o método variacional (Capítulo 3), e a estratégia de observação indireta (Capítulo 5) que utiliza o método de Newton adaptado para a observação parcial no passo 1 do Algoritmo 5.1. A avaliação é feita com base em apenas 130 problemas dos 10.000 do conjunto de problemas testes devido ao tempo de CPU despendido pela estratégia de observação indireta com o método de Newton adaptado. Além disso, a avaliação é apresentada de acordo com as classes definidas para o nível de MS-estabilizabilidade.

\section{Classe 1}

Para esta classe tem-se que o método variacional resolveu, ACP, 91,03\% dos 78 problemas e $8,97 \%$ não foram resolvidos porque a norma do segundo momento foi maior que a tolerância adotada $\left(\|X(k)\|>10^{150}\right)$. A estratégia de observação indireta, utilizando o método de Newton, resolveu, ACP, 58,98\% do total, 20,51\% não atenderam o critério de parada e 20,51\% não forma resolvidos devido a $\|X(k)\|>10^{150}(15,38 \%)$ ou a $\left\|\nabla \mathcal{J}_{T}\right\|>10^{150}(5,13 \%)$. A Figura 6.18 ilustra estes dados graficamente. 


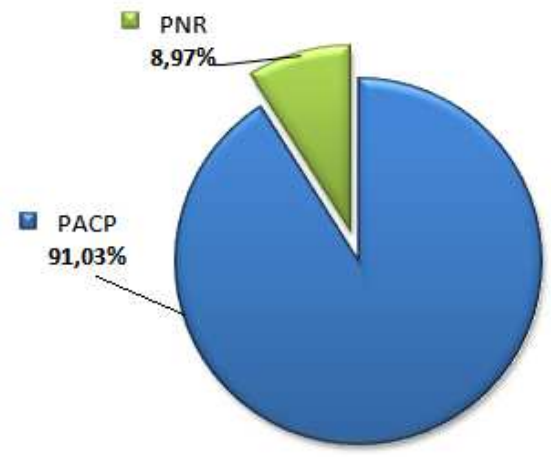

(a) Método Variacional

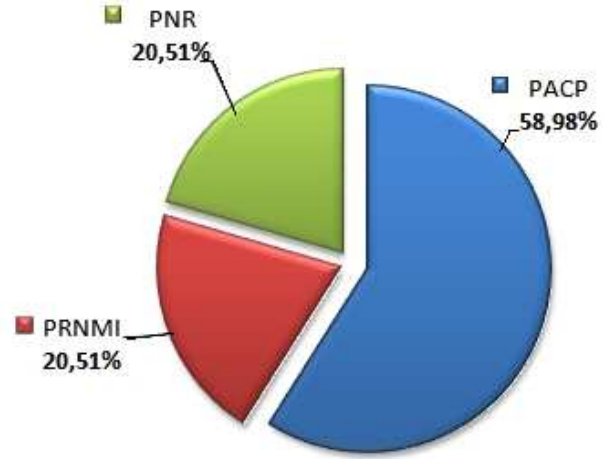

(b) Observação Indireta (MN)

Figura 6.18: Resultados obtidos por cada método, na Classe 1

Analisando somente os problemas que o MV resolveu ACP, tem-se que 64,78\% também foram resolvidos ACP pela EOI (MN) e 22,54\% não atenderam o critério de parada. Os problemas não resolvidos pela EOI (MN) representam um total de 12,68\%, sendo que $8,45 \%$ não foram resolvidos devido a $\|X(k)\|>10^{150}$ e $4,23 \%$ devido a $\left\|\nabla \mathcal{J}_{T}\right\|>10^{150}$. Para os problemas que a EOI (MN) resolveu ACP, tem-se que $100 \%$ também foram resolvidos ACP pelo MV. Na Figura 6.19 são ilustrados estes dados.

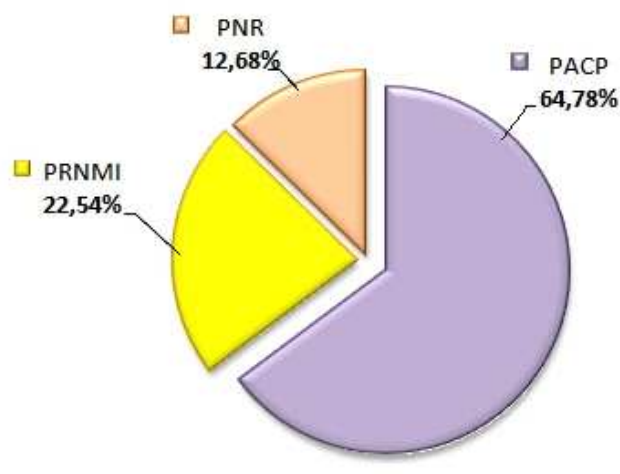

(a) Método Variacional

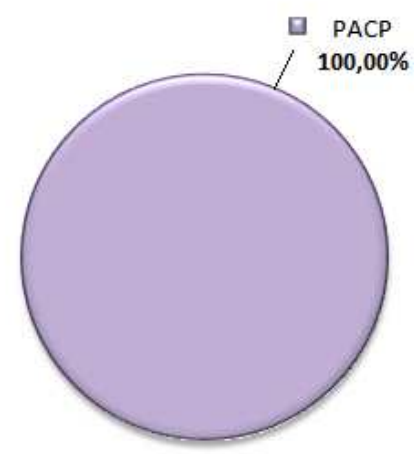

(b) Observação Indireta (MN)

Figura 6.19: Comparação dos resultados de cada método, para a Classe 1

Na Figura 6.20 está apresentado o comportamento do custo de T estágios obtido pelo $\mathrm{MV}, \mathcal{J}_{M V}$, em relação ao obtido pela EOI (MN), $\mathcal{J}_{E O I}$, sendo que uma suposição é formulada a respeito da média da razão entre os custos obtidos pelo métodos no Teste de Hipótese 6.9. 


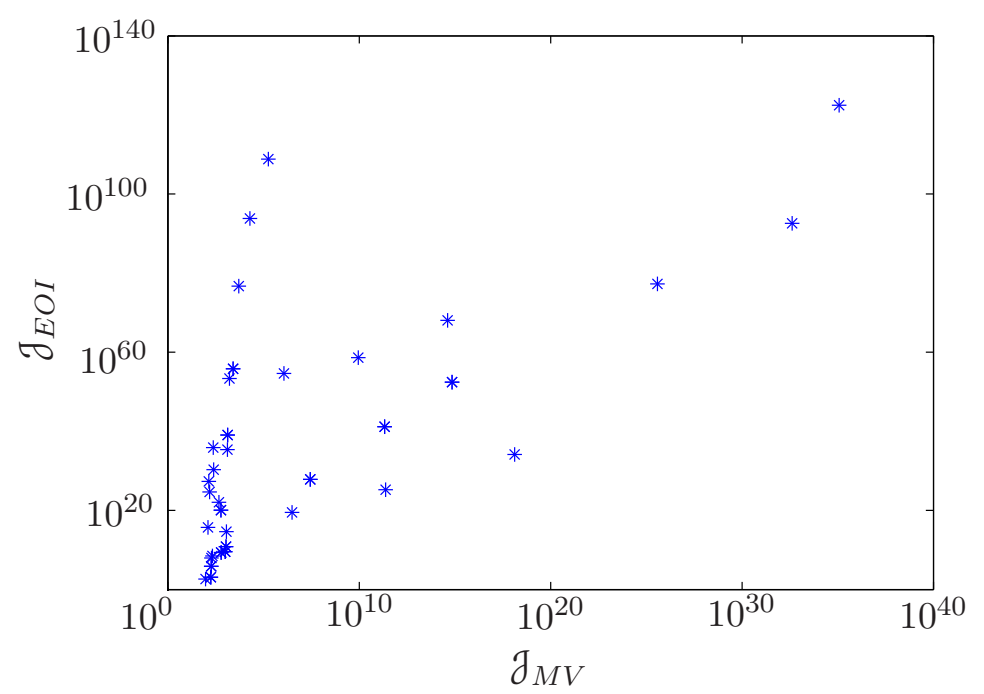

Figura 6.20: Custo do MV versus o da EOI (MN), para a Classe 1

Teste de Hipótese 6.9. Deseja-se testar se a média $\mu$, razão entre custo em escala logarítmica do $M V$ e da EOI (MN), é igual a 1, contra a alternativa de ser maior que 1. Inferimos através das amostras (custos obtidos) que o desvio padrão do custo é dado por $\sigma \approx 0,1776$. As duas hipóteses sobre a média da amostra são denotadas por $H_{0}$ (Hipótese Nula) e $H_{a}$ (Hipótese Alternativa), respectivamente. Assim,

$$
\begin{aligned}
& H_{0}: \mu=1 \quad\left(\mathcal{J}_{M V} \leq \mathcal{J}_{E O I}\right) \\
& H_{a}: \mu>1 \quad\left(\mathcal{J}_{M V}>\mathcal{J}_{E O I}\right) .
\end{aligned}
$$

O erro ao rejeitar a hipótese $H_{0}$ quando, na realidade, $H_{0}$ é verdadeira é dado por

$$
\alpha=P\left[\text { rejeitar } H_{0} \mid H_{0} \text { verdadeira }\right] \approx P[Z>2,6736] \approx 0,52 \%,
$$

sendo que $Z=\frac{\bar{X}-\mu}{S}$ e $S=\frac{\sigma}{\sqrt{46}}$ (estamos aproximando a distribuição de $Z$ por uma $t$ de student pois o número de amostras é pequeno) e considerando o valor crítico $x_{c}=1,07$. $O$ valor obtido para a média foi $\mu=0,2473$, de forma que $\mu<x_{c}$ implica em aceitar a Hipótese Nula e concluir que os métodos são equivalentes. A probabilidade de estarmos enganados nesta conclusão é de $\alpha=0,52 \%$.

Na Figura 6.21 está apresentado o comportamento do tempo de CPU obtido pelo $\mathrm{MV}, T_{M V}$, em relação ao obtido pela EOI (MN), $T_{E O I}$. Além disso, no Teste de Hipótese 6.10 é formulada uma suposição a respeito da média da razão entre os tempos obtidos pelo métodos. 


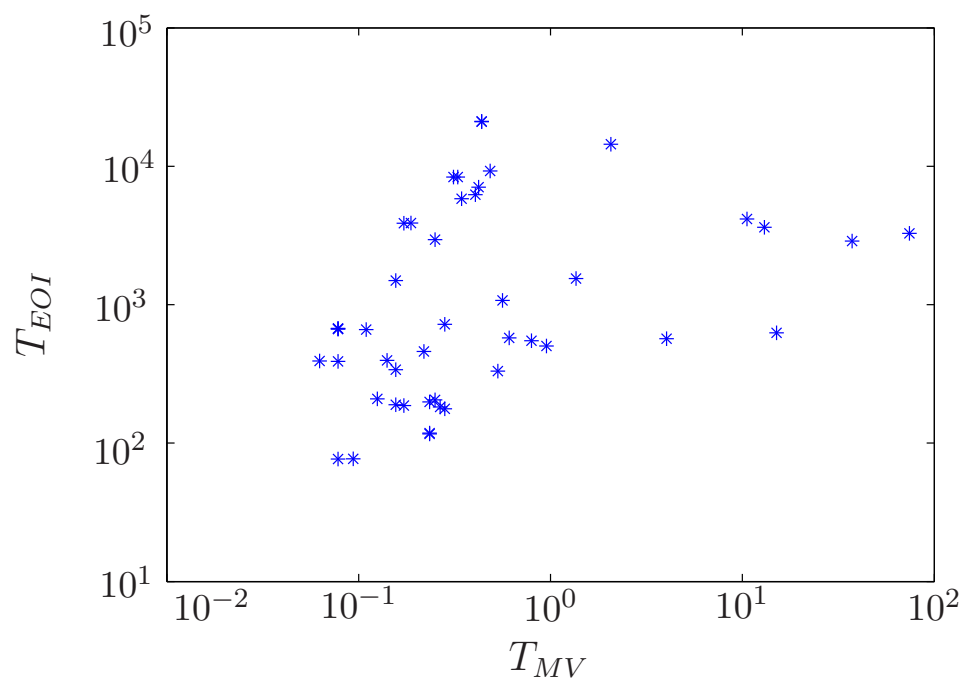

Figura 6.21: Tempo de CPU do MV versus o da EOI (MN), para a Classe 1

Teste de Hipótese 6.10. Deseja-se testar se a média $\mu$, razão entre tempo de CPU do MV e o tempo da EOI (MN), é igual a 0,006, contra a alternativa de ser maior que 0,006 . Inferimos através das amostras (tempos obtidos) que o desvio padrão é dado por $\sigma \approx 0,0051$. As duas hipóteses sobre a média da amostra são denotadas por $H_{0}$ e $H_{a}$, respectivamente. Assim,

$$
\begin{aligned}
& H_{0}: \mu=0,006\left(T_{E O I}=166 T_{M V}\right) \\
& H_{a}: \mu>0,006\left(T_{E O I}>166 T_{M V}\right) .
\end{aligned}
$$

$O$ erro ao rejeitar a hipótese $H_{0}$ quando, na realidade, $H_{0}$ é verdadeira é dado por

$$
\alpha=P\left[\text { rejeitar } H_{0} \mid H_{0} \text { verdadeira }\right] \approx P[Z>4,0178] \approx 0,011 \%,
$$

sendo que $Z=\frac{\bar{X}-\mu}{S}$ e $S=\frac{\sigma}{\sqrt{46}}$ (estamos aproximando a distribuição de $Z$ por uma $t$ de student pois o número de amostras é pequeno) e considerando o valor crítico $x_{c}$ igual a 0,009. O valor obtido para a média foi $\mu=0,0021$, de forma que $\mu<x_{c}$ implica em aceitar a Hipótese Nula e concluir que o tempo de CPU da EOI (MN) é igual a 166 vezes o tempo de CPU do MV. A probabilidade de estarmos enganados nesta conclusão é de $\alpha=0,011 \%$.

\section{Classe 2}

Para esta classe tem-se que o método variacional resolveu, ACP, $75 \%$ dos 52 problemas, sendo que o restante, $25 \%$, não foram resolvidos porque a norma do segundo momento foi maior que a tolerância adotada. A estratégia de observação indireta, 
utilizando o método de Newton, resolveu ACP 23,08\% do total, 17,30\% não atenderam o critério de parada e 59,62\% não foram resolvidos devido a $\|X(k)\|>10^{150}(29,81 \%)$ ou a $\left\|\nabla \partial_{T}\right\|>10^{150}(29,81 \%)$. A Figura 6.22 ilustra estes dados graficamente.

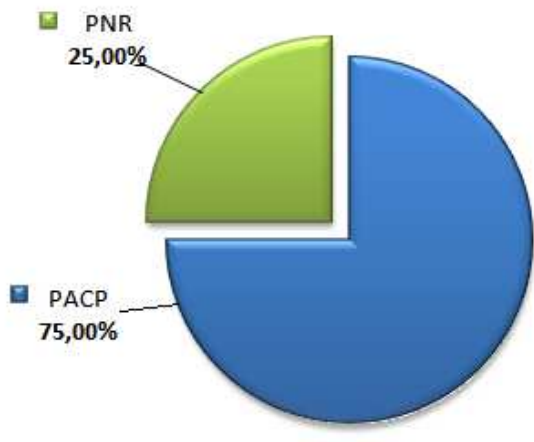

(a) Método Variacional

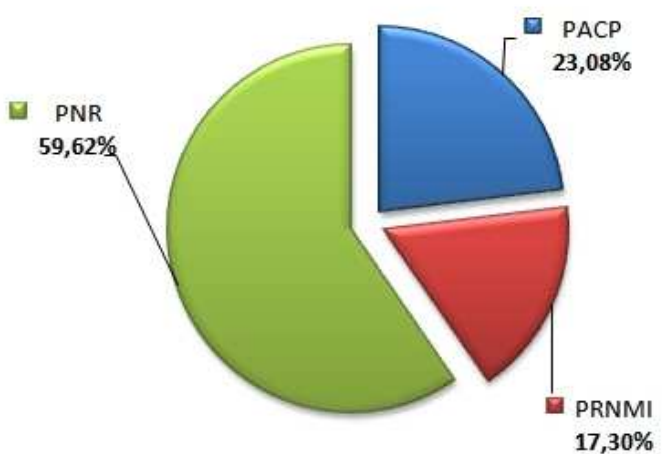

(b) Observação Indireta (MN)

Figura 6.22: Resultados obtidos por cada método, para a Classe 2

Analisando somente os problemas que o MV resolveu ACP, tem-se que 30,77\% também foram resolvidos ACP pela EOI (MN) e 23,08\% não atenderam o critério de parada. Os problemas não resolvidos pela EOI (MN) representam um total de 46,15\%, sendo que 20,51\% não foram resolvidos devido a $\|X(k)\|>10^{150}$ e $25,64 \%$ devido a $\left\|\nabla \mathcal{J}_{T}\right\|>10^{150}(37,93 \%)$. Para os problemas que a EOI (MN) resolveu ACP, tem-se que $100 \%$ também foram resolvidos ACP pelo MV. Na Figura 6.23 são ilustrados estes dados.

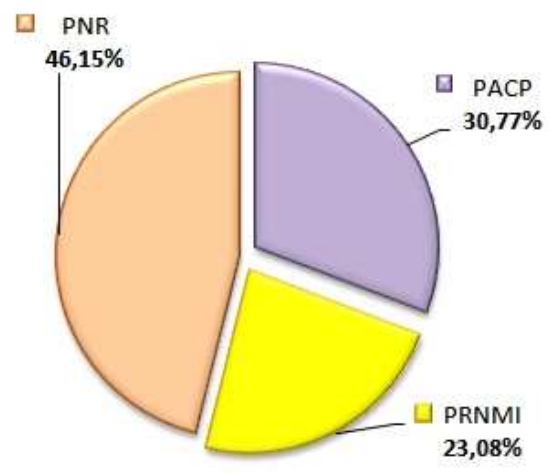

(a) Método Variacional

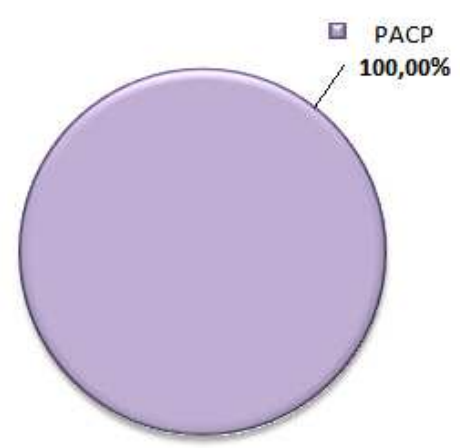

(b) Observação Indireta (MN)

Figura 6.23: Comparação dos resultados de cada método, para a Classe 2 
Na Figura 6.24 está apresentado o comportamento do custo de T estágios obtido pelo MV, $\mathcal{J}_{M V}$, em relação ao obtido pela EOI (MN), $\mathcal{J}_{E O I}$, sendo que uma suposição é formulada a respeito da média da razão entre os custos obtidos pelo métodos no Teste de Hipótese 6.11.

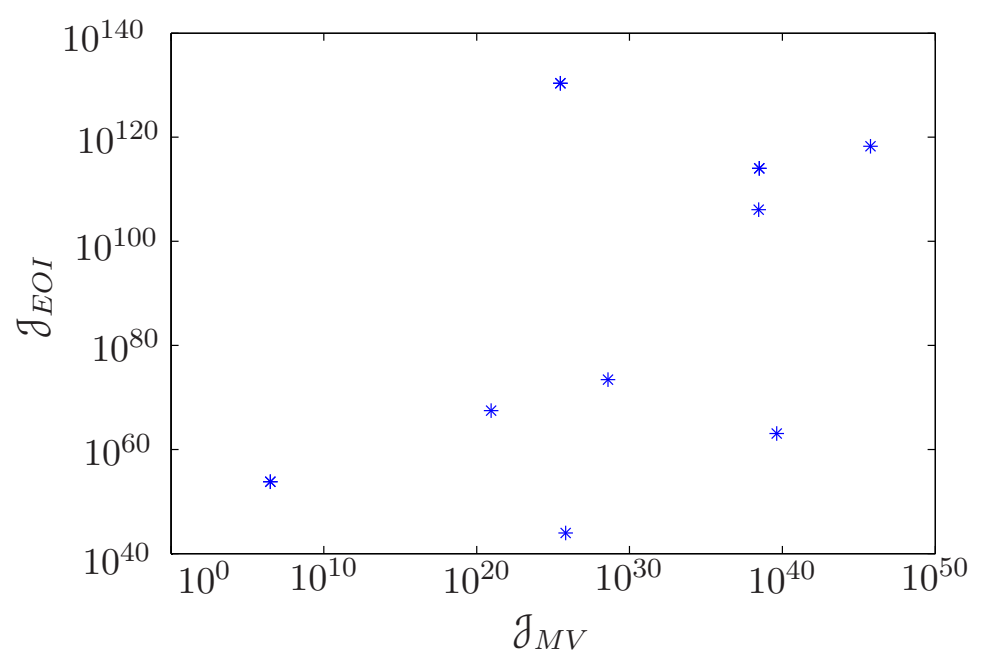

Figura 6.24: Custo do MV versus o da EOI (MN), para a Classe 2

Teste de Hipótese 6.11. Deseja-se testar se a média $\mu$, razão entre custo em escala logarítmica do MV e da EOI (MN), é igual a 1, contra a alternativa de ser maior que 1. Inferimos através das amostras (custos obtidos) que o desvio padrão do custo é dado por $\sigma \approx 0,1616$. As duas hipóteses sobre a média da amostra são denotadas por $H_{0}$ (Hipótese Nula) e $H_{a}$ (Hipótese Alternativa), respectivamente. Assim,

$$
\begin{array}{ll}
H_{0}: \mu=1 & \left(\mathcal{J}_{M V} \leq \mathcal{J}_{E O I}\right) \\
H_{a}: \mu>1 & \left(\mathcal{J}_{M V}>\mathcal{J}_{E O I}\right) .
\end{array}
$$

$O$ erro ao rejeitar a hipótese $H_{0}$ quando, na realidade, $H_{0}$ é verdadeira é dado por

$$
\alpha=P\left[\text { rejeitar } H_{0} \mid H_{0} \text { verdadeira }\right] \approx P[Z>1,0717] \approx 15,34 \%,
$$

sendo que $Z=\frac{\bar{X}-\mu}{S}$ com $Z \sim(0,1)$ e $S=\frac{\sigma}{\sqrt{12}}$ (estamos aproximando a distribuição de $Z$ por uma $t$ de student pois o número de amostras é pequeno) e considerando o valor crítico $x_{c}=1,05$. O valor obtido para a média foi $\mu=0,3311$, de forma que $\mu<x_{c}$ implica em aceitar a Hipótese Nula e concluir que os métodos são equivalentes. A probabilidade de estarmos enganados nesta conclusão é de $\alpha=15,34 \%$.

Na Figura 6.25 está apresentado o comportamento do tempo de CPU obtido pelo MV, $T_{M V}$, em relação ao obtido pela EOI (MN), $T_{E O I}$. Além disso, no Teste de 
Hipótese 6.12 é formulada uma suposição a respeito da média da razão entre os tempos obtidos pelo métodos.

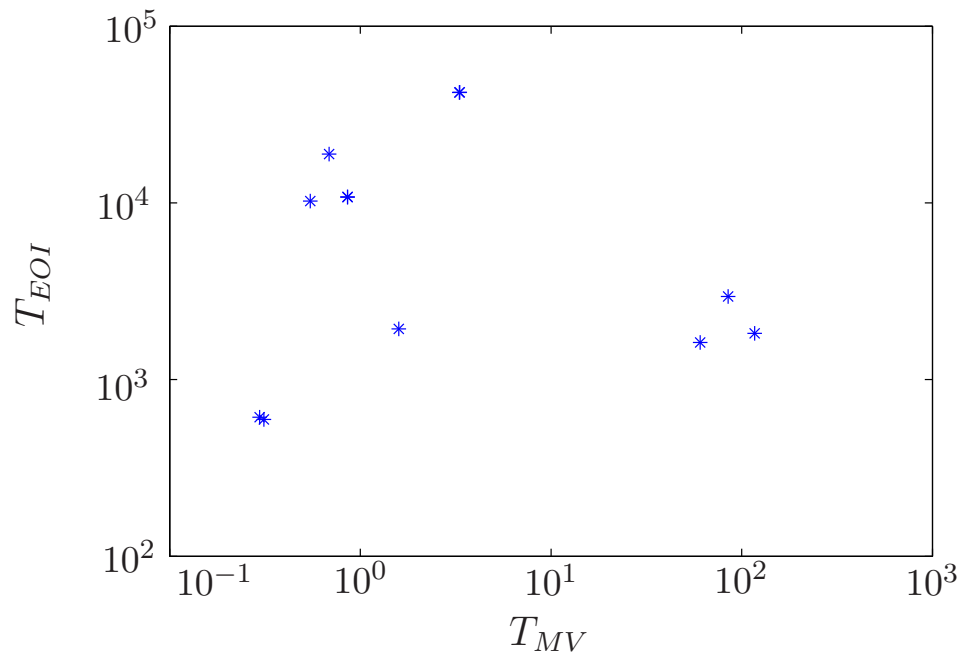

Figura 6.25: Tempo de CPU do MV versus o da EOI (MN), para a Classe 2

Teste de Hipótese 6.12. Deseja-se testar se a média $\mu$, razão entre tempo de CPU do MV e o tempo da EOI (MN), é igual a 0,003, contra a alternativa de ser maior que 0,003. Inferimos através das amostras (tempos obtidos) que o desvio padrão é dado por $\sigma \approx 0,0210$. As duas hipóteses sobre a média da amostra são denotadas por $H_{0}$ e $H_{a}$, respectivamente. Assim,

$$
\begin{aligned}
& H_{0}: \mu=0,003\left(T_{E O I}=333 T_{M V}\right) \\
& H_{a}: \mu>0,003\left(T_{E O I}>333 T_{M V}\right) .
\end{aligned}
$$

$O$ erro ao rejeitar a hipótese $H_{0}$ quando, na realidade, $H_{0}$ é verdadeira é dado por

$$
\alpha=P\left[\text { rejeitar } H_{0} \mid H_{0} \text { verdadeira }\right] \approx P[Z>1,1530] \approx 13,67 \%
$$

sendo que $Z=\frac{\bar{X}-\mu}{S}$ com $Z \sim N(0,1)$ e $S=\frac{\sigma}{\sqrt{12}}$ (estamos aproximando a distribuição de $Z$ por uma $t$ de student pois o número de amostras é pequeno) e considerando o valor crítico $x_{c}$ igual a 0,01 . O valor obtido para a média foi $\mu=0,011$, de forma que $\mu>x_{c}$ implica em rejeitar a Hipótese Nula e concluir que o tempo de CPU da EOI (MN) é maior que 333 vezes o tempo de CPU do MV. A probabilidade de estarmos enganados nesta conclusão é de $\alpha=13,67 \%$. 


\subsubsection{Considerações Finais}

Nesta seção foram apresentados os resultados obtidos a partir dos experimentos computacionais realizados, comparando os métodos desenvolvidos com o método variacional. A seguir, é apresentada uma avaliação destes resultados.

Analisando os dados obtidos para o MV e a EOI (MV), Seção 6.2.1, pode-se concluir que a EOI (MV) resolve um percentual menor de problemas, utilizando um tempo computacional maior quando comparada com o MV (veja os Testes de Hipóteses 6.2 e 6.4). No entanto, para uma parcela significativa de problemas a EOI (MV) obtém custos menores (veja os Testes de Hipóteses 6.1 e 6.3), sendo que as possíveis justificativas são as seguintes:

- o MV não tem um bom desempenho em situações em que é muito difícil, numericamente, encontrar uma sequência de ganhos inicial com custos relativamente baixos;

- as propriedades estruturais dos SLSM podem tornar o problema mais tratável pela EOI (MV). Por exemplo, se a dependência do sistema e do custo em relação ao nível de observação for "simples", é provável que os ganhos ótimos no cenário sem observação da cadeia sejam próximos dos ganhos com observação completa; em situações assim, a EOI (MV) tem um desempenho muito bom;

- a EOI (MV) é mais complexa e executa mais iterações que o MV, ou seja, obtémse um menor custo devido ao maior número de iterações realizadas.

Além disso, os resultados sugerem que a parcela de soluções que apresenta custos menores aumenta quando os problemas considerados são menos MS-estabilizáveis, sugerindo que a primeira explicação acima é a que tem maior probabilidade de estar correta.

Para os resultados obtidos pelo MV e o MN, Seção 6.2.2, tem-se que o MN resolve um percentual menor de problemas, sendo que este percentual diminui quando são considerados os problemas "pouco estáveis". Uma possível justificativa para este fato está na dificuldade de se determinar uma sequência de ganhos para inicializar o algoritmo, visto que a convergência deste método depende da sequência inicial de ganhos (veja Exemplos 4.2 e 4.3).

Verifica-se também que, para uma parcela de problemas, os valores dos custos obtidos ficaram muito próximos nos dois métodos como pode ser observado nos Testes de Hipóteses 6.5 e 6.7 . 
Em relação ao tempo consumido pelos dois métodos, tem-se que o MN é mais lento que o MV (veja os Testes de Hipóteses 6.6 e 6.8). A justificativa para este caso são os cálculos das derivadas do custo.

Por fim, os resultados obtidos pelo MV e a EOI (MN), Seção 6.2.3, tem-se que a EOI (MN) resolve um percentual menor de problemas, sendo que este percentual diminui quando são considerados os problemas "pouco estáveis".

Também observa-se que, para uma parcela de problemas, os valores dos custos obtidos ficaram muito próximos nos dois métodos (veja os Testes de Hipóteses 6.9 e 6.11), indicando que os métodos são equivalentes.

Em relação ao tempo consumido pelos dois métodos, verifica-se pelos Testes de Hipóteses 6.10 e 6.12 que a EOI (MN) é muito mais lenta que o MV. 


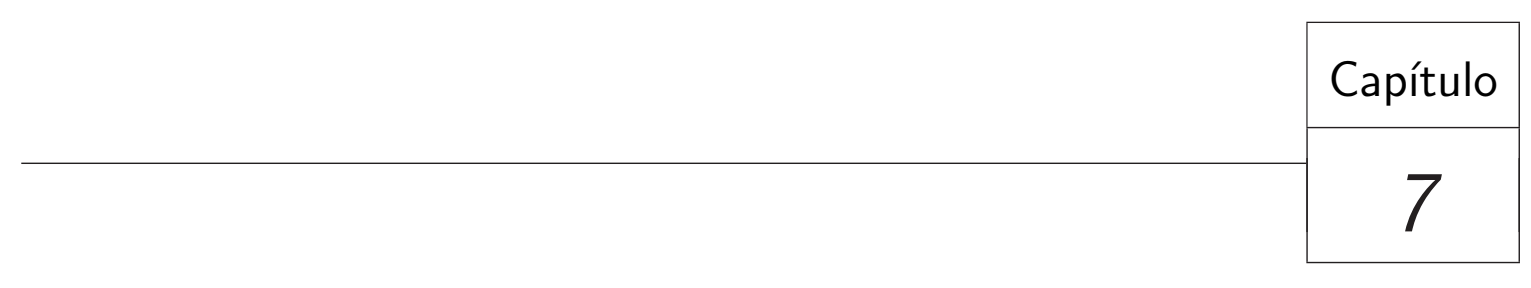

\section{Conclusão}

Este trabalho apresentou um estudo de alguns métodos de otimização utilizados para o problema do custo de $\mathrm{T}$ estágios motivado pelo problema de controle para sistemas lineares com saltos markovianos. O problema do custo médio a longo prazo consiste em determinar uma lei de controle tal que minimize o custo associado ao sistema, podendo este ser aproximado pelo custo de T estágios. Com isso, pretendeuse neste trabalho dar enfoque aos métodos de otimização aplicáveis ao problema do custo de T estágios e a uma adaptação para o problema de CMLP (Seção 5.3). Para o problema do custo de T estágios foram utilizados 3 métodos, sendo eles, o método variacional (Capítulo 3), o método de Newton (Capítulo 4) e propomos a estratégia de observação indireta (Capítulo 5), que introduz níveis intermediários de observação do estado da cadeia de Markov, partindo do cenário de observação completa e lentamente alterando para o cenário de não observação.

Para comparar o desempenho dos métodos utilizados, propomos um algoritmo capaz de elaborar um conjunto com diversos exemplos numéricos de SLSM com certas propriedades estruturais. A análise dos resultados foi realizada levando-se em consideração 2 classes de problemas, sendo que a primeira é composta pelos sistemas "bastante MS-estabilizáveis" e a segunda pelos SLSM "pouco MS-estabilizáveis", como descrito na Seção 6.2. O método variacional e a estratégia de observação indireta com o método variacional adaptado se destacaram na primeira classe, resolvendo atendendo o critério de parada mais de $50 \%$ dos problemas. Na segunda classe, o método variacional teve o melhor desempenho (62,30\% dos problema foram resolvidos ACP) e o método de Newton teve o pior desempenho $(24,22 \%)$. 
Além disso, para uma análise mais conclusiva, foram utilizados testes de hipótese para formular suposições a respeito da média do conjunto de dados obtidos. Com isso, concluiu-se que o método variacional e o de Newton são equivalentes em termos dos custos obtidos para os problemas em que o critério de parada foi satisfeito por ambos os métodos. Os testes de hipótese também mostraram que a estratégia de observação indireta com o método variacional adaptado obteve custos menores que os do método variacional (considerando os problemas resolvidos que ACP), e foram mais conclusivos para os problemas "pouco MS-estabilizáveis", sugerindo que o método variacional não tem um bom desempenho em situações em que é muito difícil, numericamente, encontrar uma sequência de ganhos inicial com custos relativamente baixos.

Assim, a estratégia de observação indireta com o método variacional adaptado pode representar uma alternativa viável para o problema considerado, principalmente para situações mais "delicadas" nas quais o sistema é menos MS-estabilizável.

Para trabalhos futuros, consideramos interessante explorar um esquema adaptativo para a estratégia de observação indireta, tanto com o método variacional adaptado quanto com o método de Newton adaptado, utilizando uma taxa variante para o decaimento do nível de observação. Com este procedimento, acreditamos ser possível obter resultados melhores, principalmente para os SLSM considerados "pouco MS-estabilizáveis". 


\section{Referências Bibliográficas}

ABADIR, K. M.; MAGNUS, J. R. (2005). Matrix Algebra (Econometric Exercises). Cambridge University Press.

BAUMEISTER, J.; LEITÃO, A. C. G. (2008). Introdução a Teoria de Controle e Programação Dinâmica. Rio de Janeiro: Instituto de Matemática Pura e Aplicada.

BAZARAA, M. S.; SHERALI, H. D.; SHETTY, C. M. (1979). Nonlinear Programming: theory and algorithmes. New York: Jonh Wiley \& Sons.

BERTSEKAS, D. P. (1987). Dynamic Programming: deterministic and stochastic model. Prentice Hall.

CHEN, C. T. (1998). Linear System Theory and Desing. Oxford University Press.

ÇINLAR, E. (1975). Introduction to Stochastic Processes. Prentice Hall College Div.

CLARKE, A. B.; DISNEY, R. L. (1970). Probability and Random Processes for Engineers and Scientists. Jonh Wiley \& Sons.

COSTA, E. F.; do VAL, J. B. R. (2002). Weak detectability and the linear quadratic control problem of discrete-time Markov jump linear systems. International Journal of Control, Special Issue on Switched and Polytopic Linear Systems, v.75, n.16-17, p. $1282-1292$.

COSTA, E. F.; MANFRIM, A. L. P.; do VAL, J. B. R. (2006). Weak controllability and weak stabilizability concepts for linear systems with Markov jump parameters. In: American Control Conference, Minneapolis, Minnesota, USA, p. 905-910.

COSTA, E. F.; VARGAS, A. N.; do VAL, J. B. R. (2011). Quadratic costs and second moments of jump linear systems with general Markov chain. Mathematics of Control, Signals and Systems, v.23, n.1, p. 141-157. 
COSTA, O. L. V.; ARAÚJO, M. V. (2008). A generalized multi-period mean-variance portfolio optimization with Markov switching parameters. Automatica, v.44, n.10, p. 2487-2497.

COSTA, O. L. V.; do VAL, J. B. R. (1998). Jump lq-optimal control for discrete-time Markovian systems with stochastic inputs. Stochastic Analysis and Applications, v.16, n.5, p. 843-858.

COSTA, O. L. V.; do VAL, J. B. R.; GEROMEL, J. C. (1999). Continuous-time state-feedback $\mathrm{H}_{2}$-control of Markovian jump linear systems via convex analysis. Automatica, v.35, n.2, p. 259 - 268.

Costa, O. L. V.; FRAGOSO, D. M.; MARQUES, R. P. (2005). Discrete-Time Markovian Jump Linear Systems. New York: Springer-Verlag.

do VAL, J. B. R.; BAŞAR, T. (1999). Receding horizon control of jump linear systems and a macroeconomic policy problem. Journal of Economic Dynamics and Control, v.23, n.8, p. 1099-1131, Aug.

FRAGOSO, M. D.; BACZYNSKI, J. (2001). Optimal Control for Continuous-Time Linear Quadratic Problems with Infinite Markov Jump Parameters. SIAM J. Control Optim., v.40, n.1, p. 270-297, Jan.

FURLONI, W. (2009). Controle por horizonte retrocedente finito com restrições de sistemas lineares discretos com saltos Markovianos. Dissertação (Mestrado), Faculdade de Engenharia Elétrica e de Computação, Universidade Estadual de Campinas, Campinas, S.P.

GUIDORIZZI, H. L. (2011). Um Curso de Cálculo, v. 1. Rio de Janeiro: LTC.

JI, Y.; CHIZECK, H. J. (1990). Controllability, stabilizability, and continuous-time Markovian jump linear quadratic control. IEEE Transactions on Automatic Control, v.35, n.7, p. $777-788$, July.

JI, Y.; CHIZECK, H. J. (1992). Jump linear quadratic Gaussian control in continuous time. IEEE Transactions on Automatic Control, v.37, n.12, p. 1884-1892, Dec.

KINCAID, D. R.; CHENEY, E. W. (1991). Numerical Analysis: mathematics of scientific computing. Brooks/Cole Publishing Company.

KIRK, E. D. (2004). Optimal Control Theory - an intoduction. Dover Publications.

LUENBERGER, D. G. (1984). Linear and Nonlinear Programming. Addison-Wesley. 
MAgalhãeS, M. N.; LIMA, A. C. P. (2002). Noções de Probabilidade e Estatística. São Paulo: Editora da Universidade de São Paulo.

MAGNUS, J. R.; NEUDECKER, H. (1985). Matrix differential calculus with applications to simple, hadamard, and kronecker products. Journal of Mathematical Psychology, v.29, n.4, p. 474-492.

MAGNUS, J. R.; NEUDECKER, H. (1999). Matrix differential calculus with applications in statistics and Econometrics. New York: Jonh Wiley \& Sons.

MANFRIM, A. L. P. (2006). O conceito de estabilizabilidade fraca para sistemas lineares com saltos Markovianos. Dissertação (Mestrado), Instituto de Ciências Matemáticas e de Computação, Universidade de São Paulo, São Carlos, S.P.

MOROZAN, T. (1995). Stability and control for linear systems with jump Markov perturbations. Stochastic Analysis and Applications, v.13, n.1, p. 91-110.

SARIDIS, G. (1983). Intelligent robotic control. IEEE Transactions on Automatic Control, v.28, n.5, p. 547-557, May.

SCHÖNEMANN, P. H. (1985). On the Formal Differentiation of Traces and Determinants. Multivariate Behavioral Research, v.20, n.2, p. 113-139.

SWORDER, D. D.; ROGERS, R. O. (1983). An lq-solution to a control problem associated with a solar thermal center receiver. IEEE Transactions on Automatic Control, v.28, n.10, p. 971-978, Oct.

VARGAS, A. N. (2004). Controle por horizonte retrocedente de sistemas lineares com saltos markovianos e ruído aditivo. Dissertação (Mestrado), Faculdade de Engenharia Elétrica e de Computação, Universidade Estadual de Campinas, Campinas, S.P.

VARGAS, A. N. (2009). Estabilidade e controle com critério de custo médio a longo prazo em sistemas lineares estocásticos. Tese (Doutorado), Faculdade de Engenharia Elétrica e de Computação, Universidade Estadual de Campinas, Campinas, S.P.

VARGAS, A. N.; COSTA, E. F.; do VAL, J. B. R. (2006). Bounds for the finite horizon cost of Markov jump linear systems with additive noise and convergence for the long run average cost. In: 45th IEEE Conference on Decision and Control, San Diego, California, USA, p. 5543-5548.

VARGAS, A. N.; do VAL, J. B. R.; COSTA, E. F. (2004). Receding horizon control of Markov jump linear system subject to noise and unobserved state chain. In: 
43rd IEEE Conference on Decision and Control, v. 4, Atlantis, Bahamas, USA, p. 4381-4386. 


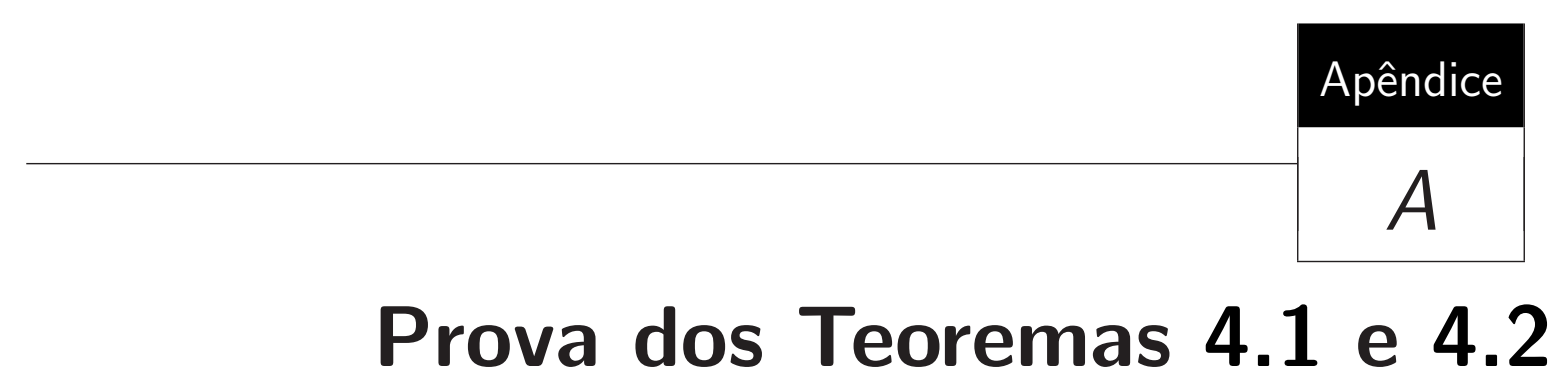

Neste apêndice são apresentados resultados sobre o cálculo diferencial matricial e a prova dos Teoremas 4.1 e 4.2 .

\section{Cálculo Diferencial Matricial}

Os resultados apresentados a seguir foram adaptados de Magnus e Neudecker (1985; 1999) e Schönemann (1985).

Definição A.1. Seja $A \in \mathcal{M}^{m, n}$ cujos elementos são funções diferenciáveis de todos os elementos de $B \in \mathcal{M}^{p, q}$. Então,

- a Matriz Jacobiana de $A$ em relação a $B$ é a matriz de dimensão $m n \times p q$, tal que

$$
\dot{A}=\frac{\partial A}{\partial B}=\frac{\partial v e c(A)}{\partial \operatorname{vec}(B)^{\prime}}
$$

- a Matriz Hessiana de $A$ em relação a $B$ é a matriz de dimensão mnpq $\times p q$, tal que

$$
\ddot{A}=\frac{\partial^{2} A}{\partial B B^{\prime}}=\frac{\partial}{\partial \operatorname{vec}(B)^{\prime}} \operatorname{vec}\left(\frac{\partial \operatorname{vec}(A)}{\partial \operatorname{vec}(B)^{\prime}}\right)^{\prime} .
$$

Proposição A.1. Considere as matrizes $A \in \mathcal{M}^{m, n}$ e $B \in \mathcal{M}^{p, q}$ então
a. $\frac{\partial A}{\partial A}=I^{m n}$
b. $\frac{\partial A^{\prime}}{\partial A}=\mathcal{P}_{m, n}$ 


$$
\begin{aligned}
& \text { c. } \frac{\partial \operatorname{Tr}\{B\}}{\partial B}=\frac{\partial \operatorname{Tr}\{B\}}{\partial \operatorname{vec}(B)^{\prime}}=\operatorname{vec}\left(I^{p}\right) ; \\
& \text { d. } \frac{\partial A^{\prime}}{\partial B}=\mathcal{P}_{m, n} \frac{\partial A}{\partial B} .
\end{aligned}
$$

Teorema A.1. Sejam $A \in \mathcal{M}^{m, r}$ e $B \in \mathcal{M}^{r, p}$ duas matrizes tais que cada um de seus elementos são funções diferenciáveis de todos os elementos da matriz $C \in \mathcal{M}^{n, q}$. Então o produto $A B$ é diferenciável e a matriz Jacobiana, de dimensão $m p \times n q$, é dada por

$$
\frac{\partial[A B]}{\partial C}=\left(B^{\prime} \otimes I^{m}\right) \frac{\partial A}{\partial C}+\left(I^{p} \otimes A\right) \frac{\partial B}{\partial C} .
$$

Teorema A.2. Sejam $A \in \mathcal{M}^{m, p}$ e $B \in \mathcal{M}^{r, s}$ duas matrizes tais que cada um de seus elementos são funções diferenciáveis de todos os elementos da matriz $C \in \mathcal{M}^{n, q}$. Então o produto de Kronecker $A \otimes B$ é diferenciável e a matriz Jacobiana, de dimensão mprs $\times n q$, é dada por

$$
\frac{\partial[A \otimes B]}{\partial C}=\left(I^{p} \otimes D\right) \frac{\partial A}{\partial C}+\left(E \otimes I^{r}\right) \frac{\partial B}{\partial C}
$$

com $D=\left(\mathcal{P}_{s, m} \otimes I^{r}\right)\left(I^{m} \otimes \operatorname{vec}(B)\right)$ e $E=\left(I^{p} \otimes \mathcal{P}_{s, m}\right)\left(\operatorname{vec}(A) \otimes I^{s}\right)$.

Teorema A.3. Sejam $A \in \mathcal{M}^{m, r}$ e $B \in \mathcal{M}^{r, p}$ duas matrizes tais que cada um de seus elementos são funções diferenciáveis de todos os elementos da matriz $C \in \mathcal{M}^{n, q}$. Então, o traço de $A B$ é diferenciável e o vetor gradiente, de dimensão $1 \times n q$, é dado por

$$
\frac{\partial \operatorname{Tr}\{A B\}}{\partial C}=\frac{\partial \operatorname{Tr}\left\{A B_{c}\right\}}{\partial C}+\frac{\partial \operatorname{Tr}\left\{A_{c} B\right\}}{\partial C}
$$

onde o subscrito c denota que a variável é considerada constante para a diferenciação.

\section{Prova do Teorema 4.1}

Demonstração. (i) Suponha que $N=1$ (para $N>1$ a prova é análoga). Tem-se que

$$
\dot{\mathcal{T}}_{\bar{A}}(V)=p_{11} \frac{\partial\left[\bar{A} V \bar{A}^{\prime}\right]}{\partial g(k)} .
$$

Aplicando os resultados sobre diferenciação matricial, obtém-se que

$$
\begin{aligned}
\frac{\partial\left[\bar{A} V \bar{A}^{\prime}\right]}{\partial g(k)} \stackrel{P . \text { A. } 1}{=}\left(\bar{A} \otimes I^{r}\right) \frac{\partial \bar{A} V}{\partial g(k)}+\left(I^{r} \otimes \bar{A} V\right) \frac{\partial \bar{A}^{\prime}}{\partial g(k)} \\
\stackrel{T . \text { A. } 1}{=}\left(\bar{A} \otimes I^{r}\right)\left[\left(V \otimes I^{r}\right) \frac{\partial \bar{A}}{\partial g(k)}+\left(I^{r} \otimes \bar{A}\right) \frac{\partial V}{\partial g(k)}\right]+\left(I^{r} \otimes \bar{A} V\right)\left(B \otimes I^{r}\right) \frac{\partial g(k)^{\prime}}{\partial g(k)} \\
\stackrel{P .2 .1, P . \mathrm{A} .1}{=}\left(\bar{A} V \otimes I^{r}\right) \frac{\partial \bar{A}}{\partial g(k)}+(\bar{A} \otimes \bar{A}) \dot{V}+(B \otimes \bar{A} V) \mathcal{P}_{s, r} \\
\stackrel{P .2 .1}{=}(\bar{A} V \otimes B)+(\bar{A} \otimes \bar{A}) \dot{V}+(B \otimes \bar{A} V) \mathcal{P}_{s, r} .
\end{aligned}
$$


Portanto,

$$
\dot{\mathcal{T}}_{\bar{A}}(V)=p_{11}\left[(\bar{A} V \otimes B)+(\bar{A} \otimes \bar{A}) \dot{V}+(B \otimes \bar{A} V) \mathcal{P}_{s, r}\right]
$$

(ii) Pela Definição A.1 e pela primeira parte do Teorema 4.1 tem-se que

$$
\frac{\partial^{2} \mathcal{T}_{\bar{A}, i}(V)}{\partial g(k) g(k)^{\prime}}=\sum_{j=1}^{N} p_{j i} \frac{\partial}{\partial g(k)}\left[\left(V_{j} \bar{A}_{j}^{\prime} \otimes B_{j}^{\prime}\right)+\dot{V}_{j}\left(\bar{A}_{j}^{\prime} \otimes \bar{A}_{j}^{\prime}\right)+\mathcal{P}_{r, s}\left(B_{j}^{\prime} \otimes V_{j} \bar{A}_{j}^{\prime}\right)\right] .
$$

Suponha que $N=1$ (para $N>1$ a prova é análoga). Aplicando os resultados sobre diferenciação matricial e as propriedades do produto de Kronecker (veja Propriedades 2.1), obtem-se que

$$
\begin{gathered}
\frac{\partial\left(V \bar{A}^{\prime} \otimes B^{\prime}\right)}{\partial g(k)} \stackrel{\text { T.A. } 2}{=} \alpha \frac{\partial\left[V \bar{A}^{\prime}\right]}{\partial g(k)}+\left(z \otimes I^{s}\right) \frac{\partial B^{\prime}}{\partial g(k)} \stackrel{B}{=} \stackrel{c t e .}{=} \alpha \frac{\partial\left[V \bar{A}^{\prime}\right]}{\partial g(k)} \\
\stackrel{\text { T.A.1, P.2.1 }}{=} \alpha\left[\left(\bar{A} \otimes I^{r}\right) \dot{V}+(B \otimes V) \mathcal{P}_{s, r}\right],
\end{gathered}
$$

onde $\alpha=\left(I^{r} \otimes\left[\mathcal{P}_{r, r} \otimes I^{s}\right)\left(I^{r} \otimes \operatorname{vec}\left(B^{\prime}\right)\right]\right)$.

$$
\begin{aligned}
\frac{\partial\left[\dot{V}\left(\bar{A}^{\prime} \otimes \bar{A}^{\prime}\right)\right]}{\partial g(k)} \stackrel{\stackrel{\text {.A.1 }}{=}\left(\bar{A} \otimes \bar{A} \otimes I^{r s}\right) \ddot{V}+\left(I^{r r} \otimes(\dot{V})^{\prime}\right) \frac{\partial\left[\bar{A}^{\prime} \otimes \bar{A}^{\prime}\right]}{\partial g(k)}}{ } \\
\stackrel{\text { T.A. } 2}{=}\left(\bar{A} \otimes \bar{A} \otimes I^{r s}\right) \ddot{V}+\left(I^{r r} \otimes(\dot{V})^{\prime}\right) \gamma\left(B \otimes I^{r}\right) \mathcal{P}_{s, r},
\end{aligned}
$$

onde $\gamma=\left(I^{r} \otimes\left[\left(\mathcal{P}_{r, r} \otimes I^{r}\right)\left(I^{r} \otimes \operatorname{vec}\left(\bar{A}^{\prime}\right)\right)\right]\right)+\left(\left[\left(I^{r} \otimes \mathcal{P}_{r, r}\right)\left(\operatorname{vec}\left(\bar{A}^{\prime}\right) \otimes I^{r}\right)\right] \otimes I^{r}\right)$.

Finalmente,

$$
\begin{aligned}
\frac{\partial\left[\mathcal{P}_{r, s}\left(B^{\prime} \otimes V \bar{A}^{\prime}\right)\right]}{\partial g(k)} & \stackrel{\text { T.A.1 }}{=}\left(B \otimes \bar{A} V \otimes I^{r s}\right) \frac{\partial \mathcal{P}_{r, s}}{\partial g(k)}+\left(I^{r r} \otimes \mathcal{P}_{r, s}\right) \frac{\partial\left[B^{\prime} \otimes V \bar{A}^{\prime}\right]}{\partial g(k)} \\
& \stackrel{\mathcal{P}}{\stackrel{\text { cte., }}{=} \text { T.A.2 } \beta \frac{\partial\left[V \bar{A}^{\prime}\right]}{\partial g(k)}} \\
& \stackrel{\text { T.A.1 }}{=}\left[\left(\bar{A} \otimes I^{r}\right) \dot{V}+\left(I^{r} \otimes V\right) \frac{\partial \bar{A}^{\prime}}{\partial g(k)}\right] \\
& =\beta\left[\left(\bar{A} \otimes I^{r}\right) \dot{V}+(B \otimes V) \mathcal{P}_{s, r}\right],
\end{aligned}
$$

onde $\beta=\left(I^{r r} \otimes \mathcal{P}_{r, s}\right)\left(\left[\left(I^{r} \otimes \mathcal{P}_{r, s}\right)\left(\operatorname{vec}\left(B^{\prime}\right) \otimes I^{r}\right)\right] \otimes I^{r}\right)$.

Portanto,

$$
\begin{aligned}
\frac{\partial^{2} \mathcal{T}_{\bar{A}, i}(V)}{\partial g(k) g(k)^{\prime}}= & p_{11}\left[(\alpha+\beta)\left(\left(\bar{A} \otimes I^{r}\right) \dot{V}+(B \otimes V) \mathcal{P}_{s, r}\right)+\right. \\
& \left.\left(\bar{A} \otimes \bar{A} \otimes I^{r s}\right) \ddot{V}+\left(I^{r r} \otimes(\dot{V})^{\prime}\right) \gamma\left(B_{j} \otimes I^{r}\right) \mathcal{P}_{s, r}\right]
\end{aligned}
$$




\section{Prova do Teorema 4.2}

Demonstração. (i) Pela Equação 2.2 tem-se que

$$
\frac{\partial J_{k}(g(k))}{\partial g(k)}=\sum_{i=1}^{N} \frac{\partial \operatorname{Tr}\left\{X_{i}(k)\left(C_{i}+g(k)^{\prime} D_{i} g(k)\right)\right\}}{\partial g(k)}
$$

Suponha que $N=1$ (para $N>1$ a prova é análoga). Aplicando os resultados sobre diferenciação matricial tem-se que

$$
\begin{gathered}
\frac{\partial \operatorname{Tr}\{X(k) C\}}{\partial g(k)} \stackrel{P .2 .1}{=} \frac{\partial\left[\operatorname{vec}\left(C^{\prime}\right)^{\prime} \operatorname{vec}(X(k))\right]}{\partial \operatorname{vec}(g(k))^{\prime}}=\operatorname{vec}(C)^{\prime} \frac{\partial \operatorname{vec}(X(k))}{\partial \operatorname{vec}(g(k))^{\prime}}=\operatorname{vec}(C)^{\prime} \dot{X}(k) \mathrm{e} \\
\frac{\partial \operatorname{Tr}\left\{X(k) g(k)^{\prime} D g(k)\right\}}{\partial g(k)} \stackrel{\text { T.A.3 }}{=} \frac{\partial \operatorname{Tr}\left\{X(k) g(k)_{c}^{\prime} D g(k)_{c}\right\}}{\partial g(k)}+\frac{\partial \operatorname{Tr}\left\{X_{c}(k) g(k)^{\prime} D g(k)\right\}}{\partial g(k)} .
\end{gathered}
$$

Finalmente,

$$
\begin{aligned}
& \frac{\partial \operatorname{Tr}\left\{X(k) g(k)_{c}^{\prime} D g(k)_{c}\right\}}{\partial g(k)} \stackrel{P .2 .1}{=} \frac{\partial\left[\operatorname{vec}\left(g(k)_{c}^{\prime} D^{\prime} g(k)_{c}\right)^{\prime} \operatorname{vec}(X(k))\right]}{\partial \operatorname{vec}(g(k))^{\prime}} \\
& \stackrel{P .2 .1, D . A .1}{=} \operatorname{vec}(D)^{\prime}(g(k) \otimes g(k)) \dot{X}(k) \mathrm{e} \\
& \frac{\left.\partial \operatorname{Tr}\left\{X(k)_{c} g(k)^{\prime} D g(k)\right)\right\}}{\partial g(k)} \stackrel{T . \text { A.3 }}{=} \frac{\partial \operatorname{Tr}\left\{X_{c}(k) g(k)^{\prime} D g(k)_{c}\right\}}{\partial g(k)}+\frac{\partial \operatorname{Tr}\left\{X_{c}(k) g(k)_{c}^{\prime} D g(k)\right\}}{\partial g(k)} \\
& =\frac{\partial \operatorname{Tr}\left\{g(k)_{c}^{\prime} D g(k) X_{c}(k)\right\}}{\partial g(k)}+\frac{\partial \operatorname{Tr}\left\{X_{c}(k) g(k)_{c}^{\prime} D g(k)\right\}}{\partial g(k)} \\
& \stackrel{P .2 .1}{=} \frac{\partial\left[\operatorname{vec}\left(g(k)_{c}\right)^{\prime} \operatorname{vec}\left(D g(k) X_{c}(k)\right)\right]}{\partial \operatorname{vec}(g(k))^{\prime}}+ \\
& \frac{\partial\left[\operatorname{vec}\left(D^{\prime} g(k)_{c} X_{c}(k)\right)^{\prime} \operatorname{vec}(g(k))\right]}{\partial \operatorname{vec}(g(k))^{\prime}} \\
& \stackrel{P .2 .1}{=} 2 \operatorname{vec}(g(k))^{\prime}(X(k) \otimes D) \frac{\partial v e c(g(k))}{\partial \operatorname{vec}(g(k))^{\prime}} \\
& \stackrel{P . A .1}{=} 2 \operatorname{vec}(g(k))^{\prime}(X(k) \otimes D) \text {. }
\end{aligned}
$$

Portanto,

$$
\frac{\partial J_{k}(g(k))}{\partial g(k)}=\left(\operatorname{vec}(C)^{\prime}+\operatorname{vec}(D)^{\prime}(g(k) \otimes g(k))\right) \dot{X}(k)+2 \operatorname{vec}(g(k))^{\prime}(X(k) \otimes D) .
$$

(ii) Pela Definição A.1 e pela primeira parte do Teorema 4.2 tem-se que

$$
\begin{aligned}
\frac{\partial^{2} J_{k}(g(k))}{\partial g(k) g(k)^{\prime}}= & \sum_{i=1}^{N} \frac{\partial}{\partial g(k)}\left[( \dot { X } _ { i } ( k ) ) ^ { \prime } \left(\operatorname{vec}\left(C_{i}\right)+\left(g(k)^{\prime} \otimes g(k)^{\prime}\right) \operatorname{vec}\left(D_{i}\right)+\right.\right. \\
& \left.2\left(X_{i}(k) \otimes D_{i}\right) \operatorname{vec}(g(k))\right]
\end{aligned}
$$


Suponha que $N=1$ (para $N>1$ a prova é análoga). Aplicando os resultados sobre diferenciação matricial e as propriedades do produto de Kronecker, obtem-se que

$$
\begin{aligned}
& \frac{\partial\left[(\dot{X}(k))^{\prime}\left(\operatorname{vec}(C)+\left(g(k)^{\prime} \otimes g(k)^{\prime}\right) \operatorname{vec}(D)\right)\right]}{\partial g(k)} \stackrel{T \cdot A .1, C \text { cte. }}{=}\left(\lambda \otimes I^{r s}\right) \ddot{X}(k)+(\dot{X}(k))^{\prime} \times \\
& \frac{\partial\left[\left(g(k)^{\prime} \otimes g(k)^{\prime}\right) \operatorname{vec}(D)\right]}{\partial g(k)} \\
& \stackrel{T . \mathrm{A} .1}{=}\left(\lambda \otimes I^{r s}\right) \ddot{X}(k)+(\dot{X}(k))^{\prime} \times \\
& \left(v e c(D)^{\prime} \otimes I^{r r}\right) \frac{\partial\left[g(k)^{\prime} \otimes g(k)^{\prime}\right]}{\partial g(k)} \\
& \stackrel{T . \text { A. }}{=}\left(\lambda \otimes I^{r s}\right) \ddot{X}(k)+(\dot{X}(k))^{\prime} \times \\
& \left(\operatorname{vec}(D)^{\prime} \otimes I^{r r}\right) \mu \text {, }
\end{aligned}
$$

onde $\lambda=\operatorname{vec}(C)^{\prime}+\operatorname{vec}(D)^{\prime}(g(k) \otimes g(k))$ e $\mu=I^{s} \otimes\left[\left(\mathcal{P}_{s, r} \otimes I^{r}\right)\left(I^{r} \otimes \operatorname{vec}\left(g(k)^{\prime}\right)\right)\right]+$ $\left[\left(I^{s} \otimes \mathcal{P}_{s, r}\right)\left(\operatorname{vec}\left(g(k)^{\prime}\right) \otimes I^{s}\right)\right] \otimes I^{r}$.

$$
\begin{aligned}
& \frac{\partial[(X(k) \otimes D) \operatorname{vec}(g(k))]}{\partial g(k)} \stackrel{T . \text { A.1 }}{=}\left(\operatorname{vec}(g(k))^{\prime} \otimes I^{r s}\right) \frac{\partial[X(k) \otimes D]}{\partial g(k)}+\left(I^{1} \otimes X(k) \otimes D\right) \times \\
& \frac{\partial[\operatorname{vec}(g(k))]}{\partial g(k)} \\
& \stackrel{T . \text { A.1 }}{=}\left(\operatorname{vec}(g(k))^{\prime} \otimes I^{r s}\right)\left(I^{r} \otimes\left[\left(\mathcal{P}^{r, s} \otimes I^{s}\right)\left(I^{r} \otimes \operatorname{vec}(D)\right)\right]\right) \times \\
& \dot{X}(k)+(X(k) \otimes D) \\
& =\tau \text {. }
\end{aligned}
$$

Portanto,

$$
\frac{\partial^{2} J_{k}(g(k))}{\partial g(k) g(k)^{\prime}}=\left(\lambda \otimes I^{r s}\right) \ddot{X}(k)+(\dot{X}(k))^{\prime}\left(\operatorname{vec}\left(D_{i}\right)^{\prime} \otimes I^{r r}\right) \mu_{i}+2 \tau .
$$



Neste apêndice é apresentado o algoritmo para determinar a Equação Recursiva de Riccati e um método, desenvolvido por Costa e do Val (2002) e Costa et al. (2006), para resolver a Equação Algébrica de Riccati Acoplada.

\section{Equação Recursiva de Riccati}

\section{Algoritmo B.1 Método para Resolver as ERR}

Passo 1: Seja $k=\{0, \ldots, T\}$ e considere $P_{i}^{(T)}=0$ para todo $i \in \mathcal{N}$.

Passo 2: Para cada $k=T-1, T-2, \ldots 0$ e $i \in \mathcal{N}$ resolva a ERR:

$$
P_{i}^{(k)}=A_{i}^{\prime} \mathscr{E}_{i}\left(P^{(k+1)}\right)\left(I-B_{i}\left(D_{i}+B_{i}^{\prime} \mathscr{E}_{i}\left(P^{(k+1)}\right) B_{i}\right)^{-1} B_{i}^{\prime} \mathscr{E}_{i}\left(P^{(k+1)}\right)\right) A_{i}+C_{i}
$$

sendo que o operador $\mathscr{E} \in \mathbb{S}^{r 0}$ é dado pela Definição 2.3.

\section{Equação Algébrica de Riccati Acoplada}

No Algoritmo B.2 é apresentado um método adaptado de Costa e do Val (2002) que testa a MS-estabilizabilidade a partir das equações algébricas de Riccati acopladas. Neste método é introduzido um parâmetro $\kappa_{i}, i \in \mathcal{N}$, que torna a matriz $\tilde{A}_{i}=\sqrt{\kappa_{i} p_{i i}} A_{i}$ estável, garantindo a existência das soluções da EARA. 


\section{Algoritmo B.2 Método para Resolver as EARAs}

Passo 1: Seja $\kappa_{i} \leq 1$ o maior escalar tal que $\tilde{A}_{i}=\sqrt{\kappa_{i} p_{i i}} A_{i}$ é estável para todo $i \in \mathcal{N}$.

Passo 2: Considere $P^{(0)}=\left(P_{1}^{(0)}, \ldots, P_{N}^{(0)}\right) \in \mathbb{S}^{r 0}$.

Passo 3: Para $k=1,2, \ldots$ e $i \in \mathcal{N}$ resolva as EARA:

$$
\begin{aligned}
-P_{i}^{(k)}+ & \kappa_{i} p_{i i} A_{i}^{\prime} P_{i}^{(k)} A_{i}+A_{i}^{\prime} \tilde{\mathscr{E}}_{i}^{(k)} A_{i}-\left(\kappa_{i} p_{i i} A_{i}^{\prime} P_{i}^{(k)} B_{i}+A_{i}^{\prime} \tilde{\mathscr{E}}_{i}^{(k)} B_{i}\right) \times \\
& \left(D_{i}+\kappa_{i} p_{i i} B_{i}^{\prime} P_{i}^{(k)} B_{i}+B_{i}^{\prime} \tilde{\mathscr{E}}_{i}^{(k)} B_{i}\right)^{-1} \times \\
& \left(\kappa_{i} p_{i i} B_{i}^{\prime} P_{i}^{(k)} A_{i}+B_{i}^{\prime} \tilde{\mathscr{E}}_{i}^{(k)} A_{i}\right)+C_{i}=0,
\end{aligned}
$$

onde $\tilde{\mathscr{E}}_{i}^{(k)}=\sum_{j \neq i} p_{i j} P_{j}^{(k)}+\left(1-\kappa_{i}\right) p_{i i} P_{i}^{(k)}$.

Proposição B.1. $P^{(k)}$ converge para algum $P \in \mathcal{S}^{r 0}$ se, e somente se $(A, B, \mathbb{P})$ é $M S$ estabilizável. 


\section{Apêndice \\ C \\ Controlabilidade e Observabilidade}

Neste apêndice é apresentado um resultado, adaptado de Chen (1998), que fornece uma forma de gerar as matrizes $\tilde{B}$ e $\tilde{C}$ (Capítulo 6).

Proposição C.1. Considere o sistema

$$
\begin{aligned}
& x_{k+1}=\tilde{A} x_{k}+\tilde{B} u_{k}, \\
& y=v x_{k},
\end{aligned}
$$

com $\tilde{A} \in \mathcal{M}^{r}, \tilde{B} \in \mathcal{M}^{r, s}$ e $v \in \mathcal{M}^{r, q}$ sendo que $\tilde{A}$ é da forma de Jordan. Suponha, por simplicidade, que a matriz $\tilde{A}$ possui dois autovalores distintos, $\lambda_{1} e \lambda_{2}$, então $\tilde{A}=\operatorname{diag}\left(\bar{A}_{1}, \bar{A}_{2}\right)$ onde o bloco $\bar{A}_{i}$ está associado a $\lambda_{i}$.

(i) Se $s=1$ então o par $(\tilde{A}, \tilde{B})$ será controlável se e somente se existir um único bloco de Jordan associado a cada autovalor distinto (ou seja, $\bar{A}_{i}=\left\{J_{1}^{(i)}\right\}$ ) e o elemento de $B$ correspondente a última linha de cada bloco de Jordan é diferente de zero.

Se $s>1$ então o par $(\tilde{A}, \tilde{B})$ será controlável se e somente se para cada $i$ o conjunto formado pelos vetores linha de $\tilde{B}$ correspondentes a última linha de $J_{j}^{(i)}, j>1$, são linearmente independentes.

(ii) Se $q=1$ então o par $(\tilde{A}, v)$ será observável se e somente se existir um único bloco de Jordan associado a cada autovalor distinto e o elemento de $v$ correspondente a primeira coluna de cada bloco de Jordan é diferente de zero.

Se $q>1$ então o par $(\tilde{A}, v)$ será observável se e somente se para cada $i$ o conjunto 
formado pelos vetores coluna de $v$ correspondentes a primeira coluna de $J_{j}^{(i)}$, $j>1$, são linearmente independentes.

Exemplo C.1. Considere os seguintes parâmetros para o sistema da Equação C.1

$$
\begin{gathered}
\tilde{A}_{1}=\left[\begin{array}{lll}
2 & 1 & 0 \\
0 & 2 & 0 \\
0 & 0 & 4
\end{array}\right], \tilde{A}_{2}=\left[\begin{array}{lll}
2 & 1 & 0 \\
0 & 2 & 0 \\
0 & 0 & 2
\end{array}\right], \tilde{B}_{1}=\left[\begin{array}{l}
0 \\
1 \\
2
\end{array}\right], \tilde{B}_{2}=\left[\begin{array}{ll}
2 & 5 \\
1 & 1 \\
2 & 2
\end{array}\right], \\
v_{1}=\left[\begin{array}{lll}
2 & 3 & 0
\end{array}\right] \text { e } v_{1}=\left[\begin{array}{lll}
1 & 3 & 0 \\
0 & 2 & 1
\end{array}\right] .
\end{gathered}
$$

A matriz $\tilde{A}_{1}$ tem dois blocos de Jordan, um de dimensão 2 associado ao autovalor 2 e outro de dimensão 1 associado ao autovalor 4 . O elemento de $\tilde{B}_{1}$ correspondente a última linha do primeiro e do segundo bloco de Jordan é igual a 1 e 2 , respectivamente. Portanto, o par $\left(\tilde{A}_{1}, \tilde{B}_{1}\right)$ é controlável. Os dois elementos de $v_{1}$ correspondentes as primeiras colunas dos blocos de Jordan são 2 e 0 . Portanto, o par $\left(\tilde{A}_{1}, v_{1}\right)$ não é observável.

A matriz $\tilde{A}_{2}$ tem dois blocos de Jordan, de dimensão 2 e 1, associado ao autovalor 2. Os vetores linhas da matriz $\tilde{B}_{2}$ que correspondem a última linha de cada bloco são [1 1] e [2 2]. Estes dois vetores não são linearmente independentes, então o par $\left(\tilde{A}_{2}, \tilde{B}_{2}\right)$ não é controlável. Os dois vetores coluna de $v_{2}$ correspondentes as primeiras colunas dos blocos de Jordan são $\left[\begin{array}{ll}1 & 0\end{array}\right]^{\prime}$ e $\left[\begin{array}{ll}0 & 1\end{array}\right]^{\prime}$. Estes vetores são linearmente independentes, portanto o par $\left(\tilde{A}_{2}, v_{2}\right)$ é observável.

Observação C.1. Pode-se mostrar que o par $(\tilde{A}, v)$ é observável (não observável) então o par $(\tilde{A}, \tilde{C})$ será observável (não observável) para $\tilde{C}=v^{\prime} v$. 


\section{Apêndice \\ D \\ Sequências de Ganhos Obtidas}

Neste apêndice são apresentadas as sequências de ganhos obtidas nos Exemplos 3.1, 4.1, 4.2, 4.3, 5.1 e 5.2. Para representar as sequências será utilizada a seguinte definição:

Definição D.1. Considere uma sequência de ganhos $\mathbf{g}=\{g(0), \ldots, g(T-1)\}$, sendo que para todo $k \in \mathcal{K}$ tem-se que $g(k) \in \mathcal{M}^{1, r}$. Define-se o operador $\mu$ tal que

$$
\mu(\mathbf{g})=\left[\begin{array}{lllll}
g(0) & \vdots & \ldots & \vdots & g(T-1)
\end{array}\right]
$$

Exemplo D.1. Seja a sequência $\mathbf{g}=\{g(0), g(1), g(2)\}$ tal que $g(0)=\left[\begin{array}{lll}5 & 1 & 3\end{array}\right]$, $g(1)=\left[\begin{array}{lll}7 & 2 & 1\end{array}\right]$ e $g(2)=\left[\begin{array}{lll}4 & 0 & 9\end{array}\right]$. Aplicando o operador $\mu$ na sequência de ganhos $\mathbf{g}$ obtém-se que

$$
\mu(\mathbf{g})=\left[\begin{array}{lllllllll}
5 & 1 & 3 & 7 & 2 & 1 & 4 & 0 & 9
\end{array}\right]
$$

\section{Exemplo 3.1}

$$
\begin{array}{rrrrrrrrrr}
\mu\left(\mathrm{g}^{*}\right) \approx[-0,9219 & 0,9380 & -0,0122 & -0,5307 & 0,2016 & -0,2136 & 0,0385 & -0,2021 \\
0,1240 & -0,6793 & 0,7163 & -0,3045 & -0,6737 & 0,5513 & -0,2624 & -0,5398 & 0,5249 \\
-0,2147 & -0,6319 & 0,6430 & -0,2778 & -0,6487 & 0,5881 & -0,2673 & -0,5933 & 0,5529 \\
-0,2490 & -0,6068 & 0,5827 & -0,2646 & -0,6174 & 0,5682 & -0,2670 & -0,5939 & 0,5410 \\
-0,2578 & -0,5922 & 0,5448 & -0,2616 & -0,5963 & 0,5415 & -0,2648 & -0,5876 & 0,5273
\end{array}
$$




$$
\begin{aligned}
& \begin{array}{lllllllll}
-0,2611 & -0,5844 & 0,5252 & -0,2616 & -0,5855 & 0,5240 & -0,2632 & -0,5824 & 0,5178
\end{array} \\
& \begin{array}{lllllllll}
-0,2619 & -0,5806 & 0,5159 & -0,2619 & -0,5807 & 0,5151 & -0,2625 & -0,5796 & 0,5127
\end{array} \\
& \begin{array}{lllllllll}
-0,2621 & -0,5788 & 0,5117 & -0,2621 & -0,5787 & 0,5112 & -0,2623 & -0,5783 & 0,5102
\end{array} \\
& \begin{array}{lllllllll}
-0,2621 & -0,5779 & 0,5098 & -0,2621 & -0,5779 & 0,5095 & -0,2622 & -0,5777 & 0,5091
\end{array} \\
& \begin{array}{lllllllll}
-0,2621 & -0,5776 & 0,5090 & -0,2621 & -0,5775 & 0,5088 & -0,2621 & -0,5774 & 0,5087
\end{array} \\
& \begin{array}{lllllllll}
-0,2621 & -0,5774 & 0,5086 & -0,2621 & -0,5774 & 0,5085 & -0,2621 & -0,5773 & 0,5085
\end{array} \\
& \begin{array}{lllllllll}
-0,2621 & -0,5773 & 0,5084 & -0,2621 & -0,5773 & 0,5084 & -0,2621 & -0,5773 & 0,5084
\end{array} \\
& \begin{array}{llllllllll}
-0,2621 & -0,5773 & 0,5084 & -0,2621 & -0,5773 & 0,5084 & -0,2621 & -0,5773 & 0,5083
\end{array} \\
& \begin{array}{lllllllll}
-0,2621 & -0,5773 & 0,5083 & -0,2621 & -0,5773 & 0,5083 & -0,2621 & -0,5773 & 0,5083
\end{array} \\
& \begin{array}{lllllllll}
-0,2621 & -0,5772 & 0,5083 & -0,2621 & -0,5772 & 0,5083 & -0,2621 & -0,5772 & 0,5083
\end{array} \\
& \begin{array}{lllllllll}
-0,2621 & -0,5772 & 0,5083 & -0,2621 & -0,5772 & 0,5083 & -0,2621 & -0,5772 & 0,5083
\end{array} \\
& \begin{array}{lllllllll}
-0,2621 & -0,5772 & 0,5083 & -0,2621 & -0,5772 & 0,5083 & -0,2621 & -0,5772 & 0,5083
\end{array} \\
& \begin{array}{lllllllll}
-0,2621 & -0,5772 & 0,5083 & -0,2621 & -0,5772 & 0,5083 & -0,2621 & -0,5772 & 0,5083
\end{array} \\
& \begin{array}{llllllllll}
-0,2621 & -0,5772 & 0,5083 & -0,2621 & -0,5772 & 0,5083 & -0,2621 & -0,5772 & 0,5083
\end{array} \\
& \begin{array}{lllllllll}
-0,2621 & -0,5772 & 0,5083 & -0,2621 & -0,5772 & 0,5083 & -0,2621 & -0,5772 & 0,5083
\end{array} \\
& \begin{array}{lllllllll}
-0,2621 & -0,5772 & 0,5083 & -0,2621 & -0,5772 & 0,5083 & -0,2621 & -0,5772 & 0,5083
\end{array} \\
& \begin{array}{lllllllll}
-0,2621 & -0,5772 & 0,5083 & -0,2621 & -0,5772 & 0,5083 & -0,2621 & -0,5772 & 0,5083
\end{array} \\
& \begin{array}{lllllllll}
-0,2621 & -0,5772 & 0,5083 & -0,2621 & -0,5772 & 0,5083 & -0,2621 & -0,5772 & 0,5083
\end{array} \\
& \begin{array}{lllllllll}
-0,2621 & -0,5772 & 0,5083 & -0,2621 & -0,5772 & 0,5083 & -0,2621 & -0,5772 & 0,5083
\end{array} \\
& \begin{array}{lllllllll}
-0,2621 & -0,5772 & 0,5083 & -0,2621 & -0,5772 & 0,5083 & -0,2621 & -0,5772 & 0,5083
\end{array} \\
& \begin{array}{lllllllll}
-0,2621 & -0,5772 & 0,5083 & -0,2621 & -0,5772 & 0,5083 & -0,2621 & -0,5772 & 0,5083
\end{array} \\
& \begin{array}{llllllllll}
-0,2621 & -0,5772 & 0,5083 & -0,2621 & -0,5772 & 0,5083 & -0,2621 & -0,5771 & 0,5083
\end{array} \\
& \begin{array}{lllllllll}
-0,2621 & -0,5771 & 0,5082 & -0,2621 & -0,5771 & 0,5082 & -0,2621 & -0,5770 & 0,5082
\end{array} \\
& \begin{array}{lllllllll}
-0,2620 & -0,5769 & 0,5081 & -0,2620 & -0,5767 & 0,5080 & -0,2620 & -0,5765 & 0,5079
\end{array} \\
& \begin{array}{lllllllll}
-0,2620 & -0,5762 & 0,5077 & -0,2619 & -0,5758 & 0,5075 & -0,2618 & -0,5751 & 0,5072
\end{array} \\
& \begin{array}{lllllllll}
-0,2617 & -0,5743 & 0,5066 & -0,2615 & -0,5733 & 0,5060 & -0,2613 & -0,5707 & 0,5052
\end{array} \\
& \begin{array}{lllllllll}
-0,2610 & -0,5682 & 0,5031 & -0,2603 & -0,5663 & 0,5008 & -0,2597 & -0,5538 & 0,4989
\end{array} \\
& \begin{array}{lllllllll}
-0,2585 & -0,5421 & 0,4893 & -0,2550 & -0,5334 & 0,4747 & -0,2494 & -0,4234 & 0,4671
\end{array} \\
& \left.\begin{array}{llll}
-0,2429 & 0 & 0 & 0
\end{array}\right] \text {. }
\end{aligned}
$$




\section{Exemplo 4.1}

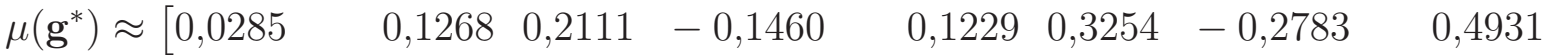

$$
\begin{aligned}
& \begin{array}{lllllllll}
0,9260 & -0,2325 & -0,0274 & 0,2805 & -0,1947 & -0,1300 & 0,1665 & -0,1837 & -0,1120
\end{array} \\
& \begin{array}{lllllllll}
0,1725 & -0,1749 & -0,1230 & 0,1601 & -0,1699 & -0,1255 & 0,1552 & -0,1674 & -0,1292
\end{array} \\
& \begin{array}{lllllllll}
0,1509 & -0,1658 & -0,1314 & 0,1479 & -0,1649 & -0,1328 & 0,1462 & -0,1644 & -0,1336
\end{array} \\
& 0,1452-0,1642 \quad-0,1340 \quad 0,1447 \quad-0,1640 \quad-0,1342 \quad 0,1444 \quad-0,1639 \quad-0,1344 \\
& 0,1443-0,1638-0,1345 \quad 0,1442 \quad-0,1638 \quad-0,1345 \quad 0,1441 \quad-0,1638 \quad-0,1345 \\
& 0,1441-0,1637 \quad-0,1345 \quad 0,1440 \quad-0,1637 \quad-0,1346 \quad 0,1440 \quad-0,1637 \quad-0,1346 \\
& 0,1440 \quad-0,1637 \quad-0,1346 \quad 0,1440 \quad-0,1637 \quad-0,1346 \quad 0,1440 \quad-0,1637 \quad-0,1346 \\
& \begin{array}{lllllllll}
0,1440 & -0,1637 & -0,1346 & 0,1440 & -0,1637 & -0,1346 & 0,1440 & -0,1637 & -0,1346
\end{array} \\
& 0,1440 \quad-0,1637 \quad-0,1346 \quad 0,1440 \quad-0,1637 \quad-0,1346 \quad 0,1440 \quad-0,1637 \quad-0,1346 \\
& \begin{array}{lllllllll}
0,1440 & -0,1637 & -0,1346 & 0,1440 & -0,1637 & -0,1346 & 0,1440 & -0,1637 & -0,1346
\end{array} \\
& 0,1440 \quad-0,1637 \quad-0,1346 \quad 0,1440 \quad-0,1637 \quad-0,1346 \quad 0,1440 \quad-0,1637 \quad-0,1346 \\
& \begin{array}{lllllllll}
0,1440 & -0,1637 & -0,1346 & 0,1440 & -0,1637 & -0,1346 & 0,1440 & -0,1637 & -0,1346
\end{array} \\
& 0,1440 \quad-0,1637 \quad-0,1346 \quad 0,1440 \quad-0,1637 \quad-0,1346 \quad 0,1440 \quad-0,1637 \quad-0,1346 \\
& \begin{array}{lllllllll}
0,1440 & -0,1637 & -0,1346 & 0,1440 & -0,1637 & -0,1346 & 0,1440 & -0,1637 & -0,1346
\end{array} \\
& \begin{array}{lllllllll}
0,1440 & -0,1637 & -0,1346 & 0,1440 & -0,1637 & -0,1346 & 0,1440 & -0,1637 & -0,1346
\end{array} \\
& \begin{array}{lllllllll}
0,1440 & -0,1637 & -0,1346 & 0,1440 & -0,1637 & -0,1346 & 0,1440 & -0,1637 & -0,1346
\end{array} \\
& \begin{array}{lllllllll}
0,1440 & -0,1637 & -0,1346 & 0,1440 & -0,1637 & -0,1346 & 0,1440 & -0,1637 & -0,1346
\end{array} \\
& \begin{array}{lllllllll}
0,1440 & -0,1637 & -0,1346 & 0,1440 & -0,1637 & -0,1346 & 0,1440 & -0,1637 & -0,1346
\end{array} \\
& 0,1440 \quad-0,1637 \quad-0,1346 \quad 0,1440 \quad-0,1637 \quad-0,1346 \quad 0,1440 \quad-0,1637 \quad-0,1346 \\
& \begin{array}{lllllllll}
0,1440 & -0,1637 & -0,1346 & 0,1440 & -0,1637 & -0,1346 & 0,1440 & -0,1637 & -0,1346
\end{array} \\
& 0,1440-0,1637 \quad-0,1346 \quad 0,1440 \quad-0,1637 \quad-0,1346 \quad 0,1440 \quad-0,1637 \quad-0,1346 \\
& \begin{array}{lllllllll}
0,1440 & -0,1637 & -0,1346 & 0,1440 & -0,1637 & -0,1346 & 0,1440 & -0,1637 & -0,1346
\end{array} \\
& \begin{array}{lllllllll}
0,1440 & -0,1637 & -0,1346 & 0,1440 & -0,1637 & -0,1346 & 0,1440 & -0,1637 & -0,1346
\end{array} \\
& \begin{array}{lllllllll}
0,1440 & -0,1637 & -0,1346 & 0,1440 & -0,1637 & -0,1346 & 0,1440 & -0,1637 & -0,1346
\end{array} \\
& 0,1440] \text {. }
\end{aligned}
$$




\section{Exemplo 4.2}

\begin{tabular}{|c|c|c|c|c|c|c|c|}
\hline$\mu\left(\mathbf{g}^{(3)}\right) \approx$ & $-0,0055$ & $0,0534 \quad 0,0$ & $83-0,4$ & $52-0,0$ & $-0,2610$ & 0,0223 & $-0,2918$ \\
\hline$-0,5317$ & 0,0861 & 0,8113 & $-0,0292$ & $-0,2423$ & 0,8238 & $-0,0392$ & $-0,3561$ \\
\hline 0,7536 & $-0,0276$ & $-0,3101$ & 0,8142 & 0,0625 & $-0,2698$ & 0,8611 & 0,1324 \\
\hline$-0,2322$ & 0,9096 & 0,1934 & 1,1536 & 3,7921 & 1,8307 & 0,3523 & 1,7841 \\
\hline 0,7477 & 0,0154 & 1,2564 & 0,4622 & $-0,1631$ & 0,9650 & 0,3156 & $-0,1975$ \\
\hline 0,8874 & 0,2858 & $-0,2066$ & 0,8568 & 0,2807 & $-0,2095$ & 0,8392 & 0,2814 \\
\hline$-0,2082$ & 0,8308 & 0,2862 & $-0,2006$ & 0,8352 & 0,2975 & $-0,1802$ & 0,8654 \\
\hline 0,3224 & $-0,1325$ & 0,9513 & 0,3745 & $-0,0220$ & 1,1511 & 0,4762 & 0,1986 \\
\hline 1,4792 & 0,6190 & 1,0194 & 1,6372 & 1,0268 & 8,4694 & 17,9682 & $-3,5019$ \\
\hline 0,3292 & $-19,6465$ & 6,4049 & 1,9997 & 3,6242 & 0,1970 & 1,7846 & 2,4665 \\
\hline 0,0170 & 6,0104 & $-16,1993$ & $-4,4811$ & 2,4439 & $-1,8844$ & $-1,0009$ & 1,8227 \\
\hline$-0,0548$ & $-0,4323$ & 1,5297 & 0,5879 & $-0,1604$ & 1,4723 & 1,2547 & $-0,0608$ \\
\hline 1,3697 & 1,3766 & 0,0131 & 1,1838 & 1,2912 & 0,1863 & 1,0853 & 1,3648 \\
\hline 0,2920 & 1,0122 & 1,4671 & 0,3750 & 0,9553 & 1,5794 & 0,4409 & 0,9116 \\
\hline 1,6897 & 0,4907 & 0,8772 & 1,7976 & 0,5300 & 0,8524 & 1,8874 & 0,5570 \\
\hline 0,8329 & 1,9649 & 0,5781 & 0,8159 & 2,0271 & 0,5969 & 0,7989 & 2,0563 \\
\hline 0,6133 & 0,7802 & 2,0536 & 0,6301 & 0,7615 & 2,0220 & 0,6460 & 0,7460 \\
\hline 1,9727 & 0,6592 & 0,7360 & 1,9147 & 0,6684 & 0,7331 & 1,8551 & 0,6718 \\
\hline 0,7389 & 1,7961 & 0,6682 & 0,7603 & 1,7386 & 0,6527 & 0,8069 & 1,7170 \\
\hline 0,6127 & 0,9546 & 1,8986 & 0,4736 & 1,0734 & 1,8219 & 0,3510 & 0,8897 \\
\hline 0,9507 & 0,5569 & 0,9873 & 0,6463 & 0,4786 & 1,1650 & 0,1723 & 0,3341 \\
\hline 1,5381 & $-0,6713$ & 0,0148 & 2,6634 & $-3,1182$ & $-0,9709$ & \multicolumn{2}{|c|}{$56,8823-121,4706$} \\
\hline$-48,4876$ & 0,7723 & 1,4401 & 0,5135 & 0,6375 & 1,7244 & 0,6313 & 0,5867 \\
\hline 1,8261 & 0,6764 & 0,5616 & 1,8722 & 0,6989 & 0,5457 & 1,8991 & 0,7137 \\
\hline 0,5350 & 1,9149 & & & & & & \\
\hline
\end{tabular}




\section{Exemplo 4.3}

$$
\begin{aligned}
& \mu\left(\mathbf{g}^{*}\right) \approx\left[\begin{array}{lllllllll}
-0,0055 & 0,0534 & 0,0183 & -0,4952 & -0,0570 & -0,2610 & 0,0223 & -0,2918
\end{array}\right. \\
& \begin{array}{lllllllll}
-0,5317 & 0,0861 & 0,8113 & -0,0292 & -0,2423 & 0,8238 & -0,0392 & -0,3561 & 0,7536
\end{array} \\
& \begin{array}{lllllllll}
-0,0276 & -0,3103 & 0,8138 & 0,0623 & -0,2732 & 0,8537 & 0,1287 & -0,2490 & 0,8738
\end{array} \\
& \begin{array}{lllllllll}
0,1751 & -0,2336 & 0,8810 & 0,2077 & -0,2243 & 0,8797 & 0,2306 & -0,2192 & 0,8727
\end{array} \\
& \begin{array}{lllllllll}
0,2463 & -0,2171 & 0,8618 & 0,2567 & -0,2171 & 0,8483 & 0,2631 & -0,2188 & 0,8331
\end{array} \\
& \begin{array}{lllllllll}
0,2664 & -0,2215 & 0,8170 & 0,2675 & -0,2248 & 0,8005 & 0,2669 & -0,2286 & 0,7842
\end{array} \\
& \begin{array}{lllllllll}
0,2652 & -0,2326 & 0,7684 & 0,2627 & -0,2366 & 0,7532 & 0,2596 & -0,2405 & 0,7390
\end{array} \\
& \begin{array}{lllllllll}
0,2563 & -0,2442 & 0,7258 & 0,2529 & -0,2476 & 0,7136 & 0,2496 & -0,2509 & 0,7025
\end{array}
\end{aligned}
$$

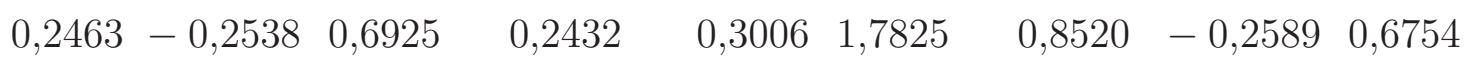

$$
\begin{aligned}
& \begin{array}{lllllllll}
0,2377 & -0,2611 & 0,6682 & 0,2353 & -0,2630 & 0,6619 & 0,2331 & -0,2647 & 0,6562
\end{array} \\
& \begin{array}{lllllllll}
0,2311 & -0,2662 & 0,6513 & 0,2294 & -0,2676 & 0,6469 & 0,2278 & -0,2687 & 0,6431
\end{array} \\
& \begin{array}{lllllllll}
0,2264 & -0,2698 & 0,6398 & 0,2252 & -0,2707 & 0,6369 & 0,2241 & -0,2715 & 0,6344
\end{array} \\
& \begin{array}{lllllllll}
0,2231 & -0,2721 & 0,6322 & 0,2223 & -0,2727 & 0,6302 & 0,2216 & -0,2733 & 0,6286
\end{array} \\
& \begin{array}{lllllllll}
0,2209 & -0,2737 & 0,6271 & 0,2204 & -0,2741 & 0,6259 & 0,2199 & -0,2744 & 0,6248
\end{array} \\
& \begin{array}{lllllllll}
0,2195 & -0,2747 & 0,6239 & 0,2191 & -0,2750 & 0,6231 & 0,2188 & -0,2752 & 0,6224
\end{array} \\
& \begin{array}{lllllllll}
0,2185 & -0,2754 & 0,6218 & 0,2183 & -0,2756 & 0,6213 & 0,2181 & -0,2757 & 0,6209
\end{array} \\
& \begin{array}{lllllllll}
0,2179 & -0,2758 & 0,6205 & 0,2178 & -0,2759 & 0,6202 & 0,2176 & -0,2760 & 0,6199
\end{array} \\
& \begin{array}{lllllllll}
0,2175 & -0,2761 & 0,6197 & 0,2174 & -0,2762 & 0,6195 & 0,2173 & -0,2762 & 0,6193
\end{array} \\
& \begin{array}{lllllllll}
0,2173 & -0,2763 & 0,6191 & 0,2172 & -0,2763 & 0,6190 & 0,2171 & -0,2763 & 0,6189
\end{array} \\
& \begin{array}{lllllllll}
0,2171 & -0,2764 & 0,6188 & 0,2171 & -0,2764 & 0,6187 & 0,2170 & -0,2764 & 0,6186
\end{array} \\
& \begin{array}{lllllllll}
0,2170 & -0,2764 & 0,6186 & 0,2170 & -0,2764 & 0,6185 & 0,2170 & -0,2765 & 0,6185
\end{array} \\
& 0,2169-0,2765 \quad 0,6185 \quad 0,2169-0,2765 \quad 0,6184 \quad 0,2169] \text {. }
\end{aligned}
$$

\section{Exemplo 5.1}

$\mu\left(\mathbf{F}_{\mathbf{1}}{ }^{*}\right) \approx\left[\begin{array}{llllllll}0,2455 & -0,0907 & 0,0716 & 0,4777 & -0,0526 & 0,2513 & 0,2561 & 0,6438\end{array}\right.$ $\begin{array}{llllllllll}0,1819 & 0,3400 & 0,0092 & 0,1811 & 0,2498 & 0,4325 & 0,1412 & 0,3120 & 0,1389 & 0,1710\end{array}$

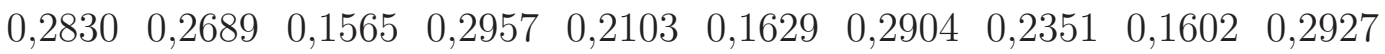
$0,2241 \quad 0,1614 \quad 0,2917 \quad 0,2291 \quad 0,1609 \quad 0,2921 \quad 0,2269 \quad 0,1611 \quad 0,2919 \quad 0,2279$ $\begin{array}{llllllllll}0,1610 & 0,2920 & 0,2275 & 0,1610 & 0,2920 & 0,2277 & 0,1610 & 0,2920 & 0,2276 & 0,1610\end{array}$ 


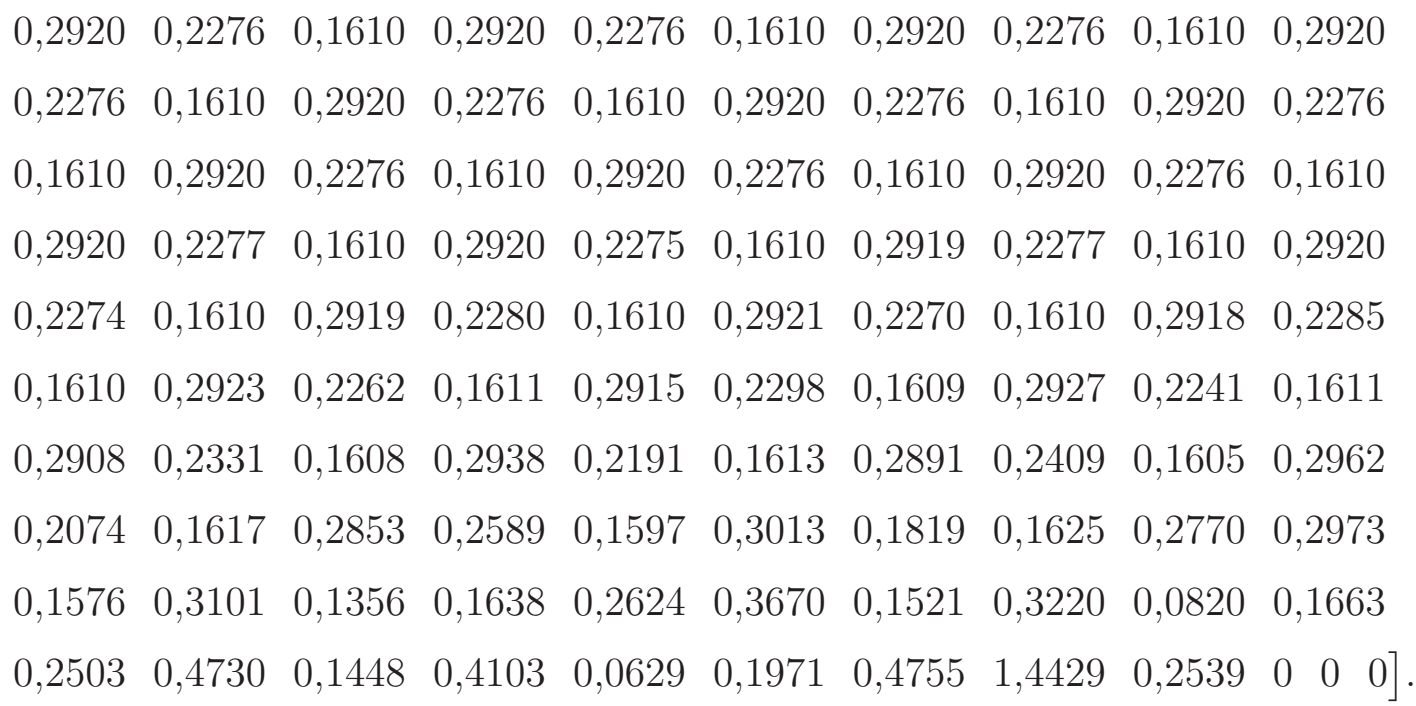
$\mu\left(\mathbf{F}_{\mathbf{2}}{ }^{*}\right) \approx\left[\begin{array}{llllllll}0,2455 & -0,0907 & 0,0716 & 0,4777 & -0,0526 & 0,2513 & 0,2561 & 0,6438\end{array}\right.$

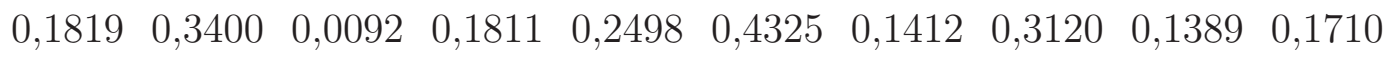
$\begin{array}{lllllllllll}0,2830 & 0,2689 & 0,1565 & 0,2957 & 0,2103 & 0,1629 & 0,2904 & 0,2351 & 0,1602 & 0,2927\end{array}$ $\begin{array}{llllllllll}0,2241 & 0,1614 & 0,2917 & 0,2291 & 0,1609 & 0,2921 & 0,2269 & 0,1611 & 0,2919 & 0,2279\end{array}$ $\begin{array}{llllllllll}0,1610 & 0,2920 & 0,2275 & 0,1610 & 0,2920 & 0,2277 & 0,1610 & 0,2920 & 0,2276 & 0,1610\end{array}$

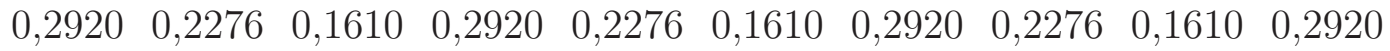

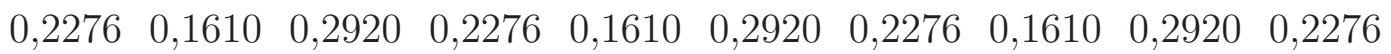
$\begin{array}{llllllllll}0,1610 & 0,2920 & 0,2276 & 0,1610 & 0,2920 & 0,2276 & 0,1610 & 0,2920 & 0,2276 & 0,1610\end{array}$ $\begin{array}{llllllllll}0,2920 & 0,2277 & 0,1610 & 0,2920 & 0,2275 & 0,1610 & 0,2919 & 0,2277 & 0,1610 & 0,2920\end{array}$ $\begin{array}{llllllllll}0,2274 & 0,1610 & 0,2919 & 0,2280 & 0,1610 & 0,2921 & 0,2270 & 0,1610 & 0,2918 & 0,2285\end{array}$ $\begin{array}{llllllllll}0,1610 & 0,2923 & 0,2262 & 0,1611 & 0,2915 & 0,2298 & 0,1609 & 0,2927 & 0,2241 & 0,1611\end{array}$ $\begin{array}{lllllllllll}0,2908 & 0,2331 & 0,1608 & 0,2938 & 0,2191 & 0,1613 & 0,2891 & 0,2409 & 0,1605 & 0,2962\end{array}$ $\begin{array}{llllllllll}0,2074 & 0,1617 & 0,2853 & 0,2589 & 0,1597 & 0,3013 & 0,1819 & 0,1625 & 0,2770 & 0,2973\end{array}$ $\begin{array}{lllllllllll}0,1576 & 0,3101 & 0,1356 & 0,1638 & 0,2624 & 0,3670 & 0,1521 & 0,3220 & 0,0820 & 0,1663\end{array}$ $\left.\begin{array}{lllllllllll}0,2503 & 0,4730 & 0,1448 & 0,4103 & 0,0629 & 0,1971 & 0,4755 & 1,4429 & 0,2539 & 0 & 0\end{array}\right]$.

\section{Exemplo 5.2}

$\mu\left(\mathbf{F}_{\mathbf{1}}{ }^{*}\right) \approx\left[\begin{array}{lllllllll}0,0161 & 0,0371 & 0,0425 & 0,0381 & -0,0516 & 0,0457 & 0,1707 & -0,1010 & 0,1489\end{array}\right.$ $\begin{array}{lllllllll}0,1724 & -0,1131 & 0,1739 & 0,1529 & -0,1127 & 0,1782 & 0,1551 & -0,1170 & 0,1857\end{array}$ $\begin{array}{lllllllll}0,1652 & -0,1216 & 0,1922 & 0,1718 & -0,1240 & 0,1954 & 0,1742 & -0,1249 & 0,1965\end{array}$ 


$$
\begin{aligned}
& \begin{array}{llllllllll}
0,1747 & -0,1250 & 0,1966 & 0,1747 & -0,1250 & 0,1966 & 0,1746 & -0,1250 & 0,1966
\end{array} \\
& \begin{array}{lllllllll}
0,1746 & -0,1250 & 0,1966 & 0,1746 & -0,1250 & 0,1966 & 0,1746 & -0,1250 & 0,1966
\end{array} \\
& \begin{array}{llllllllll}
0,1746 & -0,1250 & 0,1966 & 0,1746 & -0,1250 & 0,1966 & 0,1746 & -0,1250 & 0,1966
\end{array} \\
& \begin{array}{lllllllll}
0,1746 & -0,1250 & 0,1966 & 0,1746 & -0,1250 & 0,1966 & 0,1746 & -0,1250 & 0,1966
\end{array} \\
& \begin{array}{llllllllll}
0,1746 & -0,1250 & 0,1966 & 0,1746 & -0,1250 & 0,1966 & 0,1746 & -0,1250 & 0,1966
\end{array} \\
& \begin{array}{lllllllll}
0,1746 & -0,1250 & 0,1966 & 0,1746 & -0,1250 & 0,1966 & 0,1746 & -0,1250 & 0,1966
\end{array} \\
& \begin{array}{lllllllll}
0,1746 & -0,1250 & 0,1966 & 0,1746 & -0,1250 & 0,1966 & 0,1746 & -0,1250 & 0,1966
\end{array} \\
& \begin{array}{lllllllll}
0,1746 & -0,1250 & 0,1966 & 0,1746 & -0,1250 & 0,1966 & 0,1746 & -0,1250 & 0,1966
\end{array} \\
& \begin{array}{llllllllll}
0,1746 & -0,1250 & 0,1966 & 0,1746 & -0,1250 & 0,1966 & 0,1746 & -0,1250 & 0,1966
\end{array} \\
& \begin{array}{llllllllll}
0,1746 & -0,1250 & 0,1966 & 0,1746 & -0,1250 & 0,1966 & 0,1746 & -0,1250 & 0,1966
\end{array} \\
& \begin{array}{lllllllll}
0,1746 & -0,1250 & 0,1966 & 0,1746 & -0,1250 & 0,1966 & 0,1746 & -0,1250 & 0,1966
\end{array} \\
& \begin{array}{lllllllll}
0,1746 & -0,1250 & 0,1966 & 0,1746 & -0,1250 & 0,1966 & 0,1746 & -0,1250 & 0,1966
\end{array} \\
& \begin{array}{lllllllll}
0,1746 & -0,1250 & 0,1966 & 0,1746 & -0,1250 & 0,1966 & 0,1746 & -0,1250 & 0,1966
\end{array} \\
& 0,1746-0,1250 \quad 0,1966 \quad 0,1746 \quad-0,1250 \quad 0,1966] \text {. } \\
& \mu\left(\mathbf{F}_{\mathbf{2}}{ }^{*}\right) \approx\left[\begin{array}{lllllllll}
0,5419 & 0,1128 & 0,0959 & 0,0381 & -0,0516 & 0,0457 & 0,1707 & -0,1010 & 0,1489
\end{array}\right. \\
& \begin{array}{lllllllll}
0,1724 & -0,1131 & 0,1739 & 0,1529 & -0,1127 & 0,1782 & 0,1551 & -0,1170 & 0,1857
\end{array} \\
& \begin{array}{lllllllll}
0,1652 & -0,1216 & 0,1922 & 0,1718 & -0,1240 & 0,1954 & 0,1742 & -0,1249 & 0,1965
\end{array} \\
& \begin{array}{lllllllll}
0,1747 & -0,1250 & 0,1966 & 0,1747 & -0,1250 & 0,1966 & 0,1746 & -0,1250 & 0,1966
\end{array} \\
& \begin{array}{lllllllll}
0,1746 & -0,1250 & 0,1966 & 0,1746 & -0,1250 & 0,1966 & 0,1746 & -0,1250 & 0,1966
\end{array} \\
& \begin{array}{lllllllll}
0,1746 & -0,1250 & 0,1966 & 0,1746 & -0,1250 & 0,1966 & 0,1746 & -0,1250 & 0,1966
\end{array} \\
& \begin{array}{lllllllll}
0,1746 & -0,1250 & 0,1966 & 0,1746 & -0,1250 & 0,1966 & 0,1746 & -0,1250 & 0,1966
\end{array} \\
& \begin{array}{lllllllll}
0,1746 & -0,1250 & 0,1966 & 0,1746 & -0,1250 & 0,1966 & 0,1746 & -0,1250 & 0,1966
\end{array} \\
& \begin{array}{lllllllll}
0,1746 & -0,1250 & 0,1966 & 0,1746 & -0,1250 & 0,1966 & 0,1746 & -0,1250 & 0,1966
\end{array} \\
& \begin{array}{lllllllll}
0,1746 & -0,1250 & 0,1966 & 0,1746 & -0,1250 & 0,1966 & 0,1746 & -0,1250 & 0,1966
\end{array} \\
& 0,1746-0,1250 \quad 0,1966 \quad 0,1746 \quad-0,1250 \quad 0,1966 \quad 0,1746 \quad-0,1250 \quad 0,1966 \\
& \begin{array}{llllllllll}
0,1746 & -0,1250 & 0,1966 & 0,1746 & -0,1250 & 0,1966 & 0,1746 & -0,1250 & 0,1966
\end{array} \\
& \begin{array}{lllllllll}
0,1746 & -0,1250 & 0,1966 & 0,1746 & -0,1250 & 0,1966 & 0,1746 & -0,1250 & 0,1966
\end{array} \\
& \begin{array}{lllllllll}
0,1746 & -0,1250 & 0,1966 & 0,1746 & -0,1250 & 0,1966 & 0,1746 & -0,1250 & 0,1966
\end{array} \\
& \begin{array}{lllllllll}
0,1746 & -0,1250 & 0,1966 & 0,1746 & -0,1250 & 0,1966 & 0,1746 & -0,1250 & 0,1966
\end{array} \\
& \begin{array}{lllllllll}
0,1746 & -0,1250 & 0,1966 & 0,1746 & -0,1250 & 0,1966 & 0,1746 & -0,1250 & 0,1966
\end{array} \\
& 0,1746-0,1250 \quad 0,1966 \quad 0,1746 \quad-0,1250 \quad 0,1966] \text {. }
\end{aligned}
$$

